\title{
WestVirginiaUniversity
}

THE RESEARCH REPOSITORY @ WVU

Graduate Theses, Dissertations, and Problem Reports

2018

\section{A Study on Graph Coloring and Digraph Connectivity}

Murong Xu

Follow this and additional works at: https://researchrepository.wvu.edu/etd

\section{Recommended Citation}

Xu, Murong, "A Study on Graph Coloring and Digraph Connectivity" (2018). Graduate Theses, Dissertations, and Problem Reports. 6990.

https://researchrepository.wvu.edu/etd/6990

This Dissertation is protected by copyright and/or related rights. It has been brought to you by the The Research Repository @ WVU with permission from the rights-holder(s). You are free to use this Dissertation in any way that is permitted by the copyright and related rights legislation that applies to your use. For other uses you must obtain permission from the rights-holder(s) directly, unless additional rights are indicated by a Creative Commons license in the record and/ or on the work itself. This Dissertation has been accepted for inclusion in WVU Graduate Theses, Dissertations, and Problem Reports collection by an authorized administrator of The Research Repository @ WVU.

For more information, please contact researchrepository@mail.wvu.edu. 


\title{
A Study on Graph Coloring and Digraph Connectivity
}

\author{
Murong Xu \\ Dissertation submitted to the \\ Eberly College of Arts and Sciences \\ at West Virginia University \\ in partial fulfillment of the requirements \\ for the degree of \\ Doctor of Philosophy \\ in \\ Mathematics \\ Hong-Jian Lai, Ph.D., Chair \\ John Goldwasser, Ph.D. \\ Guodong Guo, Ph.D. \\ Rong Luo, Ph.D. \\ Jerzy Wojciechowski, Ph.D. \\ Department of Mathematics \\ Morgantown, West Virginia \\ 2018
}

Keywords: $r$-hued coloring, linear list $r$-hued coloring, edge-connectivity, restricted edge-connectivity, strong arc connectivity 


\section{ABSTRACT \\ A Study on Graph Coloring and Digraph Connectivity}

\section{Murong Xu}

This dissertation focuses on coloring problems in graphs and connectivity problems in digraphs. We obtain the following advances in both directions.

1. Results in graph coloring.

For integers $k, r>0$, a $(k, r)$-coloring of a graph $G$ is a proper coloring on the vertices of $G$ with $k$ colors such that every vertex $v$ of degree $d(v)$ is adjacent to vertices with at least $\min \{d(v), r\}$ different colors. The $r$-hued chromatic number, denoted by $\chi_{r}(G)$, is the smallest integer $k$ for which a graph $G$ has a $(k, r)$-coloring.

For a $k$-list assignment $L$ to vertices of a graph $G$, a linear $(L, r)$-coloring of a graph $G$ is a coloring $c$ of the vertices of $G$ such that for every vertex $v$ of degree $d(v), c(v) \in L(v)$, the number of colors used by the neighbors of $v$ is at least $\min \left\{d_{G}(v), r\right\}$, and such that for any two distinct colors $i$ and $j$, every component of $G\left[c^{-1}(\{i, j\})\right]$ must be a path. The linear list $r$-hued chromatic number of a graph $G$, denoted $\chi_{L, r}^{\ell}(G)$, is the smallest integer $k$ such that for every $k$-list $L, G$ has a linear $(L, r)$-coloring. Let $\operatorname{Mad}(G)$ denotes the maximum subgraph average degree of a graph $G$. We prove the following.

(i) If $G$ is a $K_{3,3}$-minor free graph, then $\chi_{2}(G) \leq 5$ and $\chi_{3}(G) \leq 10$. Moreover, the bound of $\chi_{2}(G) \leq 5$ is best possible.

(ii) If $G$ is a $P_{4}$-free graph, then $\chi_{r}(G) \leq \chi(G)+2(r-1)$, and this bound is best possible.

(iii) If $G$ is a $P_{5}$-free bipartite graph, then $\chi_{r}(G) \leq r \chi(G)$, and this bound is best possible.

(iv) If $G$ is a $P_{5}$-free graph, then $\chi_{2}(G) \leq 2 \chi(G)$, and this bound is best possible.

(v) If $G$ is a graph with maximum degree $\Delta$, then each of the following holds.

(i) If $\Delta \geq 9$ and $\operatorname{Mad}(G)<\frac{7}{3}$, then $\chi_{L, r}^{\ell}(G) \leq \max \{\lceil\Delta / 2\rceil+1, r+1\}$.

(ii) If $\Delta \geq 7$ and $\operatorname{Mad}(G)<\frac{12}{5}$, then $\chi_{L, r}^{\ell}(G) \leq \max \{\lceil\Delta / 2\rceil+2, r+2\}$.

(iii) If $\Delta \geq 7$ and $\operatorname{Mad}(G)<\frac{5}{2}$, then $\chi_{L, r}^{\ell}(G) \leq \max \{\lceil\Delta / 2\rceil+3, r+3\}$.

(vi) If $G$ is a $K_{4}$-minor free graph, then $\chi_{L, r}^{\ell}(G) \leq \max \{r,\lceil\Delta / 2\rceil\}+\lceil\Delta / 2\rceil+2$.

(vii) Every planar graph $G$ with maximum degree $\Delta$ has $\chi_{L, 2}^{\ell}(G) \leq \Delta+7$.

2. Results in digraph connectivity.

For a graph $G$, let $\kappa(G), \kappa^{\prime}(G), \delta(G)$ and $\tau(G)$ denote the connectivity, the edge-connectivity, the minimum degree and the number of edge-disjoint spanning trees of $G$, respectively. Let $f(G)$ denote $\kappa(G), \kappa^{\prime}(G)$, or $\delta(G)$, and define $\bar{f}(G)=\max \{f(H): H$ is a subgraph of $G\}$. An edge cut $X$ of a graph $G$ is restricted if $X$ does not contain all edges incident with a vertex in 
$G$. The restricted edge-connectivity of $G$, denoted by $\lambda_{2}(G)$, is the minimum size of a restricted edge-cut of $G$. We define $\overline{\lambda_{2}}(G)=\max \left\{\lambda_{2}(H): H \subseteq G\right\}$.

For a digraph $D$, let $\kappa(D), \lambda(D), \delta^{-}(D)$, and $\delta^{+}(D)$ denote the strong connectivity, arc-strong connectivity, minimum in-degree, and out-degree of $D$, respectively. For each $f \in\left\{\kappa, \lambda, \delta^{-}, \delta^{+}\right\}$, define $\bar{f}(D)=\max \{f(H): H$ is a subdigraph of $D\}$.

Catlin et al. in [Discrete Math., 309 (2009), 1033-1040] proved a characterization of $\kappa^{\prime}(G)$ in terms of $\tau(G)$. We proved a digraph version of this characterization by showing that a digraph $D$ is $k$-arc-strong if and only if for any vertex $v$ in $D, D$ has $k$-arc-disjoint spanning arborescences rooted at $v$. We also prove a characterization of uniformly dense digraphs analogous to the characterization of uniformly dense undirected graphs in [Discrete Applied Math., 40 (1992) 285 - 302].

For an integer $k>0$, a simple digraph $D$ with $|V(D)| \geq k+1$ is $k$-maximal if every subdigraph $H$ of $D$ satisfies $\lambda(H) \leq k$, but adding a new arc to $D$ results in a subdigraph $H^{\prime}$ with $\lambda\left(H^{\prime}\right) \geq k+1$. We prove that if $D$ is a simple $k$-maximal digraph on $n>k+1 \geq 2$ vertices, then

$$
|A(D)| \geq\left(\begin{array}{l}
n \\
2
\end{array}\right)+(n-1) k+\left\lfloor\frac{n}{k+2}\right\rfloor\left(1+2 k-\left(\begin{array}{c}
k+2 \\
2
\end{array}\right)\right) .
$$

This bound is best possible. Furthermore, all extremal digraphs reaching this lower bound are characterized.

Matula in [SIAM J. Appl. Math. 22 (1972) 459-480] initiated the study of $\bar{f}(G)$. He proved two minimax theorems related to $\bar{\delta}(G)$ and $\bar{\kappa}^{\prime}(G)$. We obtain analogous minimax duality results for $\overline{\lambda_{2}}(G)$. 


\section{Acknowledgements}

First and foremost, I am most indebted to my supervisor, Dr. Hong-Jian Lai, for his continued encouragement and support over these last few years. It is a pleasure to work under his supervision. Without him, this paper could not have come about.

I would like to take this opportunity to thank Dr. Suohai Fan at Jinan University (Guangzhou, China) who brought me to the research field of combinatorics and graph theory.

I would also like to thank my other committee members: Dr. John Goldwasser, Dr. Guodong Guo, Dr. Rong Luo, and Dr. Jerzy Wojciechowski, for their help during my studies.

And finally, I would like to thank the Department of Mathematics and Eberly College of Arts and Sciences at West Virginia University for providing me with an excellent study environment and support during my study as a graduate student. 


\section{DEDICATION}

To

my mother Chongyu Fu, my father Xiaoping Xu

and

my husband Xin Luo 


\section{Contents}

$\mathbf{1}$ Introduction $\quad \mathbf{1}$

1.1 Notations and Terminology . . . . . . . . . . . . . . . . . . 1

1.2 Background on Graph Coloring . . . . . . . . . . . . . . . . . 2

1.3 Background on Digraph Connectivity . . . . . . . . . . . . . 5

2 On $r$-hued Coloring of Graphs without $K_{3,3}$-minors $\quad 7$

2.1 Main Results . . . . . . . . . . . . . . . . . . . . 7

$2.2 r$-hued Chromatic Number of Graphs without $K_{3,3}$-minors . . . . . . . . . . 7

2.3 Dynamic Chromatic Number of Graphs without $K_{4}$-minors ～. . . . . . . . . . 11

3 On $r$-hued Coloring of Graphs without Short Induced Paths 14

3.1 Main Results . . . . . . . . . . . . . . . . . . . . . . . . . . . 14

3.2 On $r$-hued Colorings of $P_{4}$-free Graphs $\ldots \ldots \ldots \ldots \ldots$

3.3 On $r$-hued Colorings of $P_{5}$-free Graphs . . . . . . . . . . . . . . . . . . 18

3.3.1 On $r$-hued Colorings of $P_{5}$-free Bipartite Graphs . . . . . . . . . . . 21

3.3.2 On 2-hued Colorings of $P_{5}$-free Graphs . . . . . . . . . . . . . . . 24

3.4 Future Studies . . . . . . . . . . . . . . . . . . . . 25

4 Linear List r-hued Colorings of Graphs with Bounded Maximum Subgraph Average Degrees $\quad 26$

4.1 Main Results . . . . . . . . . . . . . . . . . . . . . 26

4.2 The Linear $r$-hued Chromatic Number of Certain Graph Families . . . . . . . . . 26

4.3 Linear 2-hued Colorings of Graphs with Bounded Average Degree . . . . . . . . . 30

5 Linear List $r$-hued Coloring of $K_{4}$-minor Free Graphs 40

5.1 Main Results . . . . . . . . . . . . . . . . . . . . . . 40

5.2 Proof of Theorem $5.1 .1 \ldots \ldots \ldots \ldots \ldots \ldots$

5.3 Proof of Theorem $5.1 .2 \ldots \ldots \ldots \ldots \ldots 4 \ldots \ldots \ldots$ 
6 Disjoint Spanning Arborescences in $k$-arc-strong Digraphs 46

6.1 Main Results . . . . . . . . . . . . . . . . . . . . . . 46

6.2 Relationship Between $k$-arc-strong Connectivity and Disjoint Spanning Arbores-

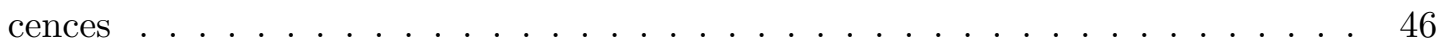

6.3 Characterization of Uniformly Dense Digraphs . . . . . . . . . . . . . 51

7 On the Lower Bound of $k$-maximal Digraphs $\quad 54$

7.1 The Problem . . . . . . . . . . . . . . . . . . . 54

7.2 Properties of $k$-maximal Digraphs f . . . . . . . . . . . . . 56

7.3 Structure of $k$-maximal Digraphs . . . . . . . . . . . . . . . . 57

7.4 The Extremal Function . . . . . . . . . . . . . . . . . . 67

8 Minimax Properties of Some Density Measures in Graphs and Digraphs 70

8.1 Introduction . . . . . . . . . . . . . . . . . . . 70

8.2 Slicing of Digraphs . . . . . . . . . . . . . . . . . . 72

8.3 A Minimax Theorem in Restricted Edge-connectivity . . . . . . . . . . . . . 74

8.3.1 Restricted Slicing of a Graph . . . . . . . . . . . . . . . . . . . 75

8.3.2 A Minimax Theorem of Restricted Edge-connectivity . . . . . . . . . . . . 77

8.4 Future Studies . . . . . . . . . . . . . . . . . . . . . 78 


\section{Chapter 1}

\section{Introduction}

\subsection{Notations and Terminology}

We consider finite simple graphs and digraphs without loops but parallel arcs. Undefined terms and notations will follow [11] for graphs and [9] for digraphs. Throughout this dissertation, $G$ denotes an (undirected) graph with vertex set $V(G)$ and edge set $E(G) ; D$ denotes a digraph with vertex set $V(D)$ and arc set $A(D) ; \Delta(G)$ and $\delta(G)$ denote the maximum degree and the minimum degree of a graph $G$, respectively.

For a graph $G$, if $X \subseteq V(G)$, then $G[X]$ is the subgraph induced by $X$. For $v \in V(G)$, let $N_{G}(v)$ denote the set of vertices adjacent to $v$ in $G$, and $d_{G}(v)=\left|N_{G}(v)\right|$. This is called the neighborhood of $v$. When $G$ is understood from this context, we often use $N(v)$ and $d(v)$ for $N_{G}(v)$ and $d_{G}(v)$, respectively. We also define $N_{G}[v]=N_{G}(v) \cup\{v\}$, which is often called the closed neighborhood of $v$. For each integer $i \geq 0$, let $D_{i}(G)$ denote the vertex set with $d_{G}(v)=i$. A vertex in $D_{i}$ is often called an $i$-vertex of $G$. For an integer $n \geq 3$, let $C_{n}$ denote a cycle on $n$ vertices and $P_{n}$ denote a path on $n$ vertices. If $S \subseteq V$ or $S \subseteq E$, then $G[S]$ is the subgraph of $G$ induced by $S$. Let $G-S=G[V(G) \backslash S]$ (if $S \subseteq V(G)$ ) or $G-S=G[E(G) \backslash S]$ (if $S \subseteq E(G)$ ). If $S \subseteq V(G)$, then let $N_{S}(v)=S \cap N_{G}(v)$. If $E(G[S])=\emptyset$, then $S$ is a stable set (or an independent set) of $G$; we also called $S$ an independent $|S|$-set. Following [11], we define a clique of a graph $G$ to be a set of mutually adjacent vertices of $G$. A clique $K$ of a graph $G$ is maximal if $K$ is not properly contained in another clique of $G$. The clique number of $G$, denoted by $\omega(G)$, is the maximum size of a clique of $G$. Let $H$ be a graph. A graph $G$ is $H$-free if $G$ does not have an induced subgraph isomorphic to $H$. In particular, a $K_{1,3}$-free graph is also called a claw-free graph. For any integer $k>0$, we denote $\bar{k}=\{1,2, \ldots, k\}$. 


\subsection{Background on Graph Coloring}

Definition 1.2.1. Let $k$ and $r$ be positive integers. A $(k, r)$-coloring of a graphs $G$ is a mapping $c: V(G) \mapsto \bar{k}$ satisfying both the following:

(C1) $c(u) \neq c(v)$, for every edge $u v \in E(G)$;

(C2) $\left|c\left(N_{G}(v)\right)\right| \geq \min \left\{d_{G}(v), r\right\}$, for every $v \in V(G)$.

The $r$-hued chromatic number of $G$, denoted by $\chi_{r}(G)$, is the smallest $k$ such that $G$ has a $(k, r)$-coloring. When $r=2, \chi_{2}(G)$ is also called the dynamic chromatic number of $G$. The study of $r$-hued colorings was initiated in [76] and [58]. Later, it was extended to 2-hued list colorings in [2]. It has now drawn lots of attention, as seen in [3], [2], [19], [24], [32], [51], [62], [67], [85], [86], among others. By the definition of $\chi_{r}(G)$, it follows immediately that $\chi(G)=\chi_{1}(G)$, and so $r$-hued is a generalization of the classical graph coloring. For any integers $i>j>0$, any $(k, i)$-coloring of $G$ is also a $(k, j)$-coloring of $G$, and so if $1 \leq j<i \leq \Delta$, then $\chi(G) \leq \chi_{j}(G) \leq \chi_{i}(G) \leq \chi_{\Delta}(G)=\chi\left(G^{2}\right)$, where $\Delta=\Delta(G)$.

In [62], it was shown that $(3,2)$-colorability remains NP-complete even when restricted to planar bipartite graphs with maximum degree at most 3 and with arbitrarily high girth. This differs considerably from the well-known result that the classical 3-colorablity is polynomially solvable for graphs with a maximum degree of at most 3. Nevertheless, there have been quite a few studies on the upper bounds of the $r$-hued chromatic number of planar graphs.

For a positive integer $h$, denote $2^{\bar{h}}$ to be the power set of $\bar{h}$. Let $L: V(G) \mapsto 2^{\bar{h}}$ be an assignment with $L(v)$ being a list of available colors at $v$. An $L$-coloring is a proper coloring $c$ such that $c(v) \in L(v)$, for every $v \in V(G)$. When such a $c$ exists, we say that $G$ is $L$-colorable. For any $v \in V(G)$, if $|L(v)|=k$, then $L$ is a $k$-element list. For a given assignment $L$ of $G$, an $(L, r)$-coloring of $G$ is an $L$-coloring, as well as an $r$-hued coloring. The list r-hued chromatic number of $G$, denoted by $\chi_{L, r}(G)$, is the least $k$ such that for every $k$-element list $L, G$ has a $(L, r)$-coloring.

The concept of linear coloring was introduced by Yuster [98]. Let $c: V(G) \mapsto \bar{k}$ be a $(k, r)$ coloring of a graph $G$. If for all $i, j \in \bar{k}$ with $i \neq j$,

$$
\text { every component of } G\left[c^{-1}(i) \cup c^{-1}(j)\right] \text { is a path, }
$$

then $c$ is a linear $(k, r)$-coloring of $G$. The linear $r$-hued chromatic number of $G$, denoted by $\chi_{r}^{\ell}(G)$, is the smallest $k$ such that $G$ admits a linear $(k, r)$-coloring. By definition, if $c$ is a linear coloring of $G$, then for each vertex $v$, every color occurs in $c\left(N_{G}(v)\right)$ at most twice, and so we always have $\chi^{\ell}(G) \geq\lceil\Delta / 2\rceil+1$. There is also a rich history of studies on linear colorings, as seen in $[22,25,33,59,65,94,95,97,98]$, among others.

Esperet et al. in [33] extended the notion to linear list colorings. The linear list chromatic number of $\mathrm{G}$, denoted by $\chi_{L}^{\ell}(G)$, is the smallest $k$ such that $G$ admits a linear $L$-coloring for every 
$k$-element list $L$. For a given assignment $L$ of $G$, a linear $(L, r)$-coloring $c$ of $G$ is an $L$-coloring as well as a linear $r$-hued coloring. The linear list $r$-hued chromatic number of $G$, denoted by $\chi_{L, r}^{\ell}(G)$, is the smallest $k$ such that for every $k$-element list $L, G$ always admits a linear $(L, r)$ coloring. It follows from the definition that $\chi_{L, r}^{\ell}(G) \geq \max \{\lceil\Delta / 2\rceil, r\}+1, \chi_{L}^{\ell}(G)=\chi_{L, 1}^{\ell}(G)$, and $\chi_{L, \Delta}^{\ell}(G)=\chi_{L}^{\ell}\left(G^{2}\right)$, where $G^{2}$ is the square graph of $G$. For any integer $i>j>0$, any linear $(L, i)$-coloring of $G$ is also a linear $(L, j)$-coloring of $G$, and so $\chi_{L}^{\ell}(G)=\chi_{L, 1}^{\ell}(G) \leq \chi_{L, 2}^{\ell}(G) \leq$ $\cdots \leq \chi_{L, \Delta}^{\ell}(G)=\chi_{L, \Delta+1}^{\ell}(G)=\cdots=\chi_{L}^{\ell}\left(G^{2}\right)$.

There have been many fascinating conjectures related to graph coloring problems. Among them are the following.

Wegner in [92] posed the following conjecture.

Conjecture 1.2.1. (Wegner [92]) If $G$ is a planar graph, then

$$
\chi_{\Delta}(G) \leq \begin{cases}\Delta(G)+5, & \text { if } 4 \leq \Delta(G) \leq 7 ; \\ \lfloor 3 \Delta(G) / 2\rfloor+1, & \text { if } \Delta(G) \geq 8 .\end{cases}
$$

For each integer $r \geq 1$, define

$$
f(r)= \begin{cases}r+3, & \text { if } 1 \leq r \leq 2 \\ r+5, & \text { if } 3 \leq r \leq 7 \\ \lfloor 3 r / 2\rfloor+1, & \text { if } r \geq 8\end{cases}
$$

Motivated by Wegner's conjecture, Song et al. raised a more general one on the upper bounds of $\chi_{r}(G)$ when $G$ is a planar graph.

Conjecture 1.2.2. (Song et al. [85]) Let $G$ be a planar graph. Then we have $\chi_{r}(G) \leq f(r)$.

Thus Wegner's conjecture is a special case when $r=\Delta$.

Define

$$
K(r)= \begin{cases}r+3, & \text { if } 2 \leq r \leq 3 \\ \lfloor 3 r / 2\rfloor+1, & \text { if } r \geq 4\end{cases}
$$

Lih et al. proved the following towards Wegner's conjecture.

Theorem 1.2.1. (Lih, Wang and Zhu [66]) Let $G$ be a $K_{4}$-minor free graph. Then

$$
\chi_{\Delta}(G) \leq K(\Delta(G))
$$

Song et al. extended Theorem 1.2.1 to the following theorem.

Theorem 1.2.2. (Song et al. [85]) Let $G$ be a $K_{4}$-minor free graph with $\Delta=\Delta(G)$, and $r \geq 2$ be an integer. Then

(i) $\chi_{r}(G) \leq K(r)$.

(ii) $\chi_{L, r}(G) \leq K(r)+1$. 
When $r=1$, Conjecture 1.2.2 is justified by the four color theorem. It is of interest to study the case when $r \geq 2$ for Conjecture 1.2.2.

Theorem 1.2.3. (Chen et al. [19]) If $G$ is a planar graph, then $\chi_{2}(G) \leq 5$.

They conjectured that $\chi_{2}(G) \leq 4$ if $G$ is a connected planar graph other than $C_{5}$. Kim et al. [51] proved this conjecture by using the four color theorem.

Theorem 1.2.4. (Kuratowski's Theorem) A graph is planar if and only if it contains no subdivision of either $K_{5}$ or $K_{3,3}$.

By Kuratowski's Theorem, it is natural to consider the upper bound of 2-hued chromatic numbers of graphs having no $K_{5}$ minors or no $K_{3,3}$ minors. For a connected graph $G$ without a $K_{5}$ minor, Kim et al. proved the following theorem.

Theorem 1.2.5. (Kim, Lee and Oum [52]) If $G$ is a connected graph other than $C_{5}$ having no $K_{5}$ minor, then $\chi_{2}(G) \leq 4$.

In $[58,76]$, it has been indicated that $\chi_{2}(G)-\chi(G)$ can be arbitrarily large. It is of interest to understand, for an integer $r \geq 2$, the relationship between $\chi_{r}(G)$ and $\chi(G)$ in different families of graphs. There have been investigations on the relationship between $\chi_{r}(G)$ and $\chi(G)$ in different families of graphs. Among them are the following.

Theorem 1.2.6. (Lai et al. , Theorem 4.2 of [57]) Let $G$ be a claw free graph. Each of the following holds.

(i) $\chi_{2}(G) \leq \chi(G)+2$.

(ii) If $G$ is connected, then $\chi_{2}(G)=\chi(G)+2$ if and only if $G$ is a cycle of length 5 or an even cycle of length not a multiple of 3 .

Theorem 1.2.7. (Li and Lai [60]) Let $G$ be a claw-free graph. Then $\chi_{3}(G) \leq \max \{\chi(G)+3,7\}$. This bound is best possible.

Theorem 1.2.8. (Alishahi, Theorem 3 of [4]) Let $k \geq 35$ be an integer, and let $G$ be a k-regular $C_{4}$-free graph. Then $\chi_{2}(G) \leq \chi(G)+2\lceil 4 \ln (k)+1\rceil$.

Theorem 1.2.9. (Ahadi et al. [1]) If $G$ is a $P_{4}$-free graph, then $\chi_{2}(G) \leq \chi(G)+2$.

The maximum subgraph average degree of $G$, denoted by $\operatorname{Mad}(G)$, is defined by

$$
\operatorname{Mad}(G)=\max \left\{\frac{2|E(H)|}{|V(H)|}, H \subseteq G\right\} .
$$

It is noted that if $G$ is a planar graph, then $\operatorname{Mad}(G)<6$. Therefore, it is natural to investigate $\chi_{r}(G)$ and $\chi_{L, r}^{\ell}(G)$ for graphs $G$ with bounded values of $\operatorname{Mad}(G)$.

Song et al. in [86] proved the following theorem.

Theorem 1.2.10. (Song et al. [86]) If $r \geq 3$ and $G$ is a planar graph with $g(G) \geq 6$, then $\chi_{r}(G) \leq r+5$. 


\subsection{Background on Digraph Connectivity}

For a graph $G$, let $\kappa^{\prime}(G)$ and $\tau(G)$ denote edge-connectivity and the number of edge-disjoint spanning trees of $G$, respectively. For a digraph $D, \kappa(D)$ and $\lambda(D)$ denote the strong connectivity and the arc-strong connectivity of $D$, respectively. For any disjoint subsets $X, Y \subseteq V(D)$, define $(X, Y)_{D}$ to be the set of all $\operatorname{arcs}$ in $D$ with tail in $X$ and head in $Y$, and let

$$
\partial_{D}^{+}(X)=(X, V(D)-X)_{D} \text { and } \partial_{D}^{-}(X)=(V(D)-X, X)_{D}
$$

and let $d_{D}^{+}(X)=\left|\partial_{D}^{+}(X)\right|$ and $d_{D}^{-}(X)=\left|\partial_{D}^{-}(X)\right|$. When $X=\{v\}$, we write $d_{D}^{+}(v)=\left|\partial_{D}^{+}(\{v\})\right|$ (called the out degree of $v$ in $D$ ) and $d_{D}^{-}(v)=\left|\partial_{D}^{-}(\{v\})\right|$ (called the in degree of $v$ in $D$ ). A digraph $D$ is $k$-arc-strong if for any nonempty proper subset $X \subset V(D), d_{D}^{+}(X) \geq k$. Thus $\lambda(D)$ is the largest integer $k$ such that $D$ is $k$-arc-strong.

Catlin et al. in [16] proved a characterization of $\kappa^{\prime}(G)$ in terms of $\tau(G)$.

Theorem 1.3.1. (Catlin et al. , Theorem 1.1 of [16]) Let $G$ be a connected graph and let $k \geq 1$ be an integer. Each of the following holds.

(i) $\kappa^{\prime}(G) \geq 2 k$ if and only if $\forall X^{\prime} \subseteq E(G)$ with $\left|X^{\prime}\right| \leq k, \tau\left(G-X^{\prime}\right) \geq k$.

(ii) $\kappa^{\prime}(G) \geq 2 k+1$ if and only if $\forall X^{\prime} \subseteq E(G)$ with $\left|X^{\prime}\right| \leq k+1, \tau\left(G-X^{\prime}\right) \geq k$.

An arborescence is an oriented tree $T$ such that for some $r \in V(T)$, we have $d_{T}^{-}(r)=0$, and for any $v \in V(T)-r$, we have $d_{T}^{-}(v)=1$. The vertex $r$ is the root of $T$ and $T$ is an $r$-arborescence. Let $\tau(D)$ be the maximum number of arc-disjoint spanning arborescences in $D$. It is natural to investigate whether there is a similar relationship in digraphs. One such attempt was made in Theorem 1.4 of [16]. It is unfortunately that, due to the misunderstanding of a result of Frank (mistakenly quoted as a result of Edmonds in Theorem 3.1 of [16]), the proof of Theorem 1.4 of [16] is false. Therefore, the problem of investigating the relationship between $\lambda(D)$ and $\tau(D)$ remains unanswered.

For a graph $G$, we follow [11] to use $c(G)$ to denote the number of components of $G$. Following Cunningham [23], the strength $\eta(G)$ and fractional arboricity $\gamma(G)$ of $G$ are defined as

$$
\eta(G)=\min _{X \subseteq E(G)}\left\{\frac{|X|}{c(G-X)-c(G)}\right\} \text {, and } \gamma(G)=\max _{X \subseteq E(G)}\left\{\frac{|X|}{|V(G[X])|-c(G[X])}\right\},
$$

where the minimum and maximum are taken over all subsets $X$ such that the corresponding denominators are not zero. In [15], it has been indicated that the well-known spanning tree packing theorem of Nash-Williams [77] and Tutte [91] can be restated as the following.

Theorem 1.3.2. A nontrivial graph $G$ has $k$ edge-disjoint spanning trees if and only if $\eta(G) \geq k$.

In [15], an attempt to obtain a fractional version of Theorem 1.3.2 was made, and the following characterization was proven. 
Theorem 1.3.3. (Catlin et al. , Theorem 6 of [15]) Let $G$ be a connected graph. The following are equivalent.

(i) $\gamma(G)(|V(G)|-1)=|E(G)|$.

(ii) $\eta(G)(|V(G)|-1)=|E(G)|$.

(iii) $\eta(G)=\gamma(G)$.

(iv) For any integers $s \geq t>0$ with $\gamma(G)=\frac{s}{t}$, G has s spanning trees $T_{1}, T_{2}, \cdots, T_{s}$ such that every edge $e \in E(G)$ is in exactly $t$ members in the multiset $\left\{T_{1}, T_{2}, \cdots, T_{s}\right\}$.

(v) For any integers $s \geq t>0$ with $\eta(G)=\frac{s}{t}$, $G$ has s spanning trees $T_{1}, T_{2}, \cdots, T_{s}$ such that every edge e $\in E(G)$ is in exactly $t$ members in the multiset $\left\{T_{1}, T_{2}, \cdots, T_{s}\right\}$.

A graph $G$ satisfying Theorem 1.3.3(i) is called a uniformly dense graph. These results have been applied in [20] to give short proofs for some results on the higher-order edge toughness of a graph in [18]; and to obtain characterization of minimal graphs whose edge-connectivity equals the spanning tree packing number in [42]. The characterizations of uniformly dense graphs have also been applied to many other studies, such as cyclic base orderings [46] and the problem of packing hypertrees [41]. It is natural to consider the study on uniformly dense digraphs analogous to those characterizations in [15].

Given a graph $G$, Matula $[73,74,75]$ first studied the quantity

$$
\bar{\kappa}^{\prime}(G)=\max \left\{\kappa^{\prime}(H): H \subseteq G\right\} .
$$

He called $\bar{\kappa}^{\prime}(G)$ the strength of $G$. Mader [71] considered an extremal problem related to $\bar{\kappa}^{\prime}(G)$. For an integer $k>0$, a simple graph $G$ with $|V(G)| \geq k+1$ is $k$-maximal if $\bar{\kappa}^{\prime}(G) \leq k$ but for any edge $e \in E\left(G^{c}\right), \bar{\kappa}^{\prime}(G+e)>k$. In [71], Mader proved the following.

Theorem 1.3.4. (Mader [71]) If $G$ is a k-maximal graph on $n>k \geq 1$ vertices, then

$$
|E(G)| \leq(n-k) k+\left(\begin{array}{c}
k \\
2
\end{array}\right) .
$$

Furthermore, this bound is best possible.

It has been noted that being a $k$-maximal graph requires a certain level of edge density. Towards this direction, the following was proven in 1990.

Theorem 1.3.5. (Lai, Theorem 2 of [53]) If $G$ is a $k$-maximal graph on $n>k+1 \geq 2$ vertices, then

$$
|E(G)| \geq(n-1) k-\left(\begin{array}{c}
k \\
2
\end{array}\right)\left\lfloor\frac{n}{k+2}\right\rfloor .
$$

Furthermore, this bound is best possible.

It is natural to consider extending the theorems above to digraphs. 


\section{Chapter 2}

\section{On $r$-hued Coloring of Graphs without $K_{3,3}$-minors}

\subsection{Main Results}

The main purpose of this study is to consider the $r$-hued coloring of $K_{3,3}$-minor free graphs and $K_{4}$-minor free graphs. Without using the four color theorem, we obtain the following results.

Theorem 2.1.1. Let $G$ be a connected graph without $K_{3,3}$ minor, then $\chi_{2}(G) \leq 5$.

Theorem 2.1.2. Let $G$ be a $K_{4}$-minor free graph, then $\chi_{2}(G) \leq 4$ except for $G \cong C_{5}$.

Theorem 2.1.2 improves Theorem 1.2.2, in which every $K_{4}$-minor free graph $G$ satisfying $\chi_{2}(G) \leq 5$ is also characterized.

\section{$2.2 r$-hued Chromatic Number of Graphs without $K_{3,3}$-minors}

In this section, we first build some reduction propositions, which will be utilized to prove Theorem 2.1.1. A vertex cut $S$ of $G$ is called $t$-cut if $|S|=t$.

Definition 2.2.1. Let $G$ be a graph, and $S \subseteq V(G)$ be a t-cut of $G$. $H_{i}(i=1,2)$ are two vertex-disjoint subgraphs of $G-S$ with $G-S=H_{1} \cup H_{2}$. Let $K_{t}$ be the complete graph on vertex set $S$, and $G_{i}=G\left[V\left(H_{i}\right) \cup S\right] \cup K_{t}(i=1,2)$. We call $G$ the $t$-sum of $G_{1}$ and $G_{2}$, denoted by $G=G_{1} \oplus_{t} G_{2}$, in which edges in $K_{t}$ but not in $G$ will be deleted.

We first consider the case when $t=1$.

Proposition 2.2.2. Let $G=G_{1} \oplus_{1} G_{2}$. Then $\chi_{r}(G) \leq \max \left\{\chi_{r}\left(G_{1}\right), \chi_{r}\left(G_{2}\right), r+1\right\}$. 
Proof. Let $V\left(G_{1}\right) \cap V\left(G_{2}\right)=\{w\}$ and let $k=\max \left\{\chi_{r}\left(G_{1}\right), \chi_{r}\left(G_{2}\right), r+1\right\}$. So for $i \in\{1,2\}$, there exists a proper $(k, r)$-coloring of $G_{i}$ with $c_{i}: V\left(G_{i}\right) \mapsto \bar{k}$. Without loss of generality (w.l.o.g. in short), we can permute the color of $w$ in $G_{1}$ so that $c_{1}(w)=c_{2}(w)$. Define $c: V(G) \mapsto \bar{k}$ as

$$
c(v)= \begin{cases}c_{1}(v), & \text { if } v \in V\left(G_{1}\right) \\ c_{2}(v), & \text { if } v \in V\left(G_{2}\right)\end{cases}
$$

Then for any $v \in V(G)-\{w\}$, both hued conditions $(\mathrm{C} 1)$ and $(\mathrm{C} 2)$ are satisfied at $v$ by definition, and so $c(w) \neq c(u)$ for any $u \in N(w)$. Let $h_{1}=\left|N_{G_{1}}(w)\right|$ and $h_{2}=\left|N_{G_{2}}(w)\right|$. If $\max \left\{h_{1}, h_{2}\right\} \geq r$ or $|c(N(w))| \geq r$, then $w$ satisfies the hued condition (C2) under the coloring $c$. So we assume that $\max \left\{h_{1}, h_{2}\right\} \leq r-1$ and $|c(N(w))| \leq r-1$. Thus, $\left|c\left(N_{G_{1}}(w)\right)\right|=\left|N_{G_{1}}(w)\right|=h_{1}$ and $\left|c\left(N_{G_{2}}(w)\right)\right|=\left|N_{G_{2}}(w)\right|=h_{2}$, respectively.

Let $t=\min \left\{h_{2}, r-h_{1}\right\}$. Since $k \geq r+1$, we can choose $t$ colors $\left\{\alpha_{1}, \alpha_{2}, \ldots, \alpha_{t}\right\}$ from $\bar{k}-$ $c\left(N_{G_{1}}[w]\right)$. As $t \leq h_{2}$, we can pick $t$ vertices $\left\{u_{1}, u_{2}, \ldots, u_{t}\right\}$ from $N_{G_{2}}(w)$. Define a permutation of $V\left(G_{2}\right)$ on $\bar{k}$ by

$$
\pi=\left(\begin{array}{llllll}
c_{2}(w) & c_{2}\left(u_{1}\right) & c_{2}\left(u_{2}\right) & \ldots & c_{2}\left(u_{t}\right) & \ldots \\
c_{2}(w) & \alpha_{1} & \alpha_{2} & \ldots & \alpha_{t} & \ldots
\end{array}\right)
$$

and define a new coloring $c^{\prime}$ of $V(G)$ such that

$$
c^{\prime}(v)= \begin{cases}c_{1}(v), & \text { if } v \in V\left(G_{1}\right) \\ \pi\left(c_{2}(v)\right), & \text { if } v \in V\left(G_{2}\right)-\{w\} .\end{cases}
$$

Then $\left|c^{\prime}(w)\right|=\left|c_{G_{1}}^{\prime}(w)\right|+\left|c_{G_{2}}^{\prime}(w)\right| \geq h_{1}+t=\min \left\{h_{1}+h_{2}, r\right\}$. Thus, $c^{\prime}$ is a proper $(k, r)$-coloring of $G$. This implies the Proposition 2.2.2 holds.

By definition 2.2.1, when $G=G_{1} \oplus_{t} G_{2}, K_{t}=G_{1} \cap G_{2}$ may not be a subgraph of $G$. For our purpose, we give the different propositions for different cases.

Proposition 2.2.3. Let $G=G_{1} \oplus_{2} G_{2}$ be a 2-connected graph, where $V\left(G_{1}\right) \cap V\left(G_{2}\right)=\{x, y\}$. If $x y \in E(G)$, then each of the followings holds.

(i) Let

$$
k= \begin{cases}\max \left\{\chi_{r}\left(G_{1}\right), \chi_{r}\left(G_{2}\right)\right\}, & \text { if } 1 \leq r \leq 2 \\ \max \left\{\chi_{r}\left(G_{1}\right), \chi_{r}\left(G_{2}\right), 2 r-1\right\}, & \text { if } r \geq 3\end{cases}
$$

Then $\chi_{r}(G) \leq k$;

(ii)If $G_{2}=C_{5}$, then $\chi_{2}(G) \leq \max \left\{\chi_{2}\left(G_{1}\right), 3\right\}$. In particular, $\chi_{2}\left(C_{5} \oplus_{2} C_{5}\right)=4$.

Proof. (i) As it is well-known when $r=1, \chi(G) \leq \max \left\{\chi\left(G_{1}\right), \chi\left(G_{2}\right)\right\}$, we assume $r \geq 2$. For $k \geq \max \left\{\chi_{r}\left(G_{1}\right), \chi_{r}\left(G_{2}\right)\right\}, G_{i}$ permits a $(k, r)$-coloring $c_{i}$ for $i \in\{1,2\}$. Since $r \geq 2$, by Proposition 2.1 of [57], $k \geq 3$. Hence, for $i \in\{1,2\}$, there exists a $(k, r)$-coloring $c_{i}: V\left(G_{i}\right) \mapsto \bar{k}$ satisfying $c_{1}(x)=c_{2}(x)$ and $c_{1}(y)=c_{2}(y)$ so that

$$
\min \left\{\left|c_{i}\left(N_{G_{i}}(x)\right)\right|, r\right\}+\min \left\{\left|c_{i}\left(N_{G_{i}}(y)\right)\right|, r\right\}
$$


is maximized. Define $c: V(G) \mapsto \bar{k}$ as

$$
c(v)= \begin{cases}c_{1}(v), & \text { if } v \in V\left(G_{1}\right) \\ c_{2}(v), & \text { if } v \in V\left(G_{2}\right) .\end{cases}
$$

We denote the coloring $c$ in (2.4) by $c=c_{1} \cup c_{2}$. As $c_{1}(x)=c_{2}(x)$ and $c_{1}(y)=c_{2}(y), c$ is well-defined. Since $c_{i}$ is a $(k, r)$-coloring of $G_{i}$, we conclude that $c$ is a proper $k$-coloring of $G$ such that every vertex in $V(G)-\{x, y\}$ satisfies the hued condition $(C 2)$. If both $x$ and $y$ satisfy the hued condition $(C 2)$, then $c$ is a $(k, r)$-coloring of $G$. In the following, w.l.o.g., we argue by contradiction and always assume that $c(x)=1, c(y)=2$ and $x$ does not satisfy the hued condition $(C 2)$. For any vertex $w \in\{x, y\}$, let $D c(w)$ denote the set of colors which appears in both $N_{G_{1}}(w) \backslash\{x, y\}$ and $N_{G_{2}}(w) \backslash\{x, y\}, A c(w)$ denote the set of colors which is absent from $N_{G_{1}}[w] \cup N_{G_{2}}[w]$. By definition, $D c(x) \cap A c(x)=\emptyset$. As $x$ does not satisfy the hued condition $(C 2), 1,2 \notin D c(x) \cup A c(x)$, and $D c(x) \neq \emptyset$.

Since $x y \in E(G)$, for any $w \in\{x, y\}$, if $\max \left\{d_{G_{1}}(w), d_{G_{2}}(w)\right\} \geq r$, then $w$ satisfies the hued condition $(C 2)$ under the coloring $c=c_{1} \cup c_{2}$. Since $\kappa(G) \geq 2$, it follows by definition that $c=c_{1} \cup c_{2}$ is an $r$-hued coloring of $G$ when $r=1,2$. Now we only consider the case when $r \geq 3$, and assume that $\max \left\{d_{G_{1}}(x), d_{G_{2}}(y)\right\} \leq r-1$.

Since $k \geq r+2$ and $\max \left\{d_{G_{1}}(x), d_{G_{2}}(y)\right\} \leq r-1, A c(x) \neq \emptyset$. Pick arbitrarily an $\alpha \in A c(x)$ and a $\beta \in D c(x)$ such that $\beta \in D c(x) \cap D c(y)$ whenever $D c(x) \cap D c(y) \neq \emptyset$. If $\left|c\left(N_{G}(y)\right)\right| \geq r+1$ or $D c(x) \cap D c(y) \neq \emptyset$, permute colors $\alpha$ and $\beta$ in $c_{2}$ to get a $(k, r)$-coloring $c_{2}^{\prime}$ of $G_{2}$; if there exists one of the colors in $\{\alpha, \beta\}$ not appearing in $N_{G}(y)$, or both $\alpha$ and $\beta$ appear in the same neighborhood $N_{G_{i}}(y)$ for some $i\left(i=2\right.$, say), permute colors $\alpha$ and $\beta$ in $c_{2}$ to get a new $(k, r)$-coloring $c_{2}^{\prime}$ of $G_{2}$. In the cases above, the resulting coloring $c^{\prime}=c_{1} \cup c_{2}^{\prime}$ of $G$ satisfies $\min \left\{\left|c^{\prime}\left(N_{G}(x)\right)\right|, r\right\}+\min \left\{\left|c^{\prime}\left(N_{G}(y)\right)\right|, r\right\}>\min \left\{\left|c\left(N_{G}(x)\right)\right|, r\right\}+\min \left\{\left|c\left(N_{G}(y)\right)\right|, r\right\}$ contrary to (2.3). Hence we must have $\left|c\left(N_{G}(y)\right)\right| \leq r$ and $D c(x) \cap D c(y)=\emptyset$. Furthermore, as $\alpha$ and $\beta$ are picked arbitrarily, we have $A c(x) \cup D c(x) \subseteq c\left(N_{G}(y)\right),(A c(x) \cup D c(x)) \cap D c(y)=\emptyset$, and both $A c(x)$ and $D c(x)$ are located in such a way that one set is in $G_{1}$, another is in $G_{2}$. We have $\left|c\left(N_{G}(y)\right)\right| \geq|A c(x)|+|D c(x)|+1$. When $r=3,|A c(x)| \geq 2$; when $r \geq 4,|A c(x)| \geq r-1$. It follows that $\left|c\left(N_{G}(y)\right)\right| \geq r+1$, a contradiction.

(ii) Let $G_{2}=C_{5}=v_{1} v_{2} v_{3} v_{4} v_{5} v_{1}, V\left(G_{1}\right) \cap V\left(G_{2}\right)=\left\{v_{1}, v_{2}\right\}$. Let $k=\max \left\{\chi_{2}\left(G_{1}\right), 3\right\}, c$ be a $(k, 2)$-coloring of $G_{1}$ such that $c\left(v_{1}\right)=1, c\left(v_{2}\right)=2$. Since $\kappa(G) \geq 2$, if $\min \left\{d_{G_{1}}\left(v_{1}\right), d_{G_{1}}\left(v_{2}\right)\right\} \geq$ 2 , then both $v_{1}$ and $v_{2}$ satisfy the hued condition $(C 2)$ under $c$ which can be looked as a partial $(k, 2)$-coloring of $G$. Now we extend the coloring $c$ to $\left\{v_{3}, v_{4}, v_{5}\right\}$ by letting $c\left(v_{3}\right)=1, c\left(v_{4}\right)=3$ and $c\left(v_{5}\right)=2$. Thus, the resulting coloring $c$ is a $(k, 2)$-coloring of $G$. It is easy to check that $\chi_{2}\left(C_{5} \oplus_{2} C_{5}\right)=4$.

Proposition 2.2.4. Let $G=G_{1} \oplus_{2} G_{2}$ be a 2-connected graph, where $V\left(G_{1}\right) \cap V\left(G_{2}\right)=\{x, y\}$. 
(i)Let

$$
k= \begin{cases}\max \left\{\chi_{r}\left(G_{1}\right), \chi_{r}\left(G_{2}\right)\right\}, & \text { if } r=1 \\ \max \left\{\chi_{r}\left(G_{1}\right), \chi_{r}\left(G_{2}\right), r+3\right\}, & \text { if } r \geq 2 .\end{cases}
$$

If $d_{G_{i}}(x), d_{G_{i}}(y) \geq r(i=1,2)$, then $\chi_{r}(G) \leq k$.

(ii) When $r \geq 3, \chi_{r}(G) \leq \max \left\{\chi_{r}\left(G_{1}\right), \chi_{r}\left(G_{2}\right), 2 r+1\right\}$.

Proof. It is trivial when $r=1$. Suppose $r \geq 2$. By Proposition 2.2.3 and by the fact $d_{G_{i}}(x), d_{G_{i}}(y) \geq r(i=1,2)$ when considering $(i)$, we may assume that $x$ is not adjacent to $y$ in $G$. Since $k \geq \max \left\{\chi_{r}\left(G_{1}\right), \chi_{r}\left(G_{2}\right)\right\}$, each $G_{i}$ permits a $(k, r)$-coloring $c_{i}$. Similar to the proof of Proposition 2.2.3, there exist colorings $c_{1}$ and $c_{2}$ such that $c_{1}(x)=c_{2}(x)$ and $c_{1}(y)=c_{2}(y)$. Choose such colorings $c_{1}$ and $c_{2}$ so that (2.3) is satisfied. Define $c=c_{1} \cup c_{2}$ as in (2.4). By definition of $c, c$ is a proper $k$-coloring of $G$ such that every vertex in $V(G)-\{x, y\}$ satisfies the hued condition $(C 2)$. If both $x$ and $y$ satisfy the hued condition $(C 2)$, then $c$ is a $(k, r)$-coloring of $G$. In the following, w.l.o.g., we always assume that $x$ do not satisfy the hued condition $(C 2)$ and $c(x)=1, c(y)=2$. For any vertex $w \in\{x, y\}$, let $D c(w)$ denote the set of colors which appears in both $N_{G_{1}}(w) \backslash\{x, y\}$ and $N_{G_{2}}(w) \backslash\{x, y\}, A c(w)$ denote the set of colors which is absent from $N_{G_{1}}[w] \cup N_{G_{2}}[w]$. By definition, $D c(x) \cap A c(x)=\emptyset$. Since $k \geq r+3$ when $r \geq 2$, $D c(x) \neq \emptyset$ and $A c(x) \neq \emptyset$.

As $x$ does not satisfy the hued condition $(C 2), 1,2 \notin D c(x) \cup A c(x)$. Similar to the proof of Proposition 2.2.3, we have $\left|c\left(N_{G}(y)\right)\right| \leq r, D c(x) \cap D c(y)=\emptyset, A c(x) \cup D c(x) \subseteq c\left(N_{G}(y)\right)$, $(A c(x) \cup D c(x)) \cap D c(y)=\emptyset$, and $A c(x)$ and $D c(x)$ are located in such a way that one set is in $G_{1}$, another is in $G_{2}$. We have $\left|c\left(N_{G}(y)\right)\right| \geq|A c(x)|+|D c(x)|$.

(i) Assume that $d_{G_{i}}(x), d_{G_{i}}(y) \geq r$, for $i \in\{1,2\}$. As $c_{i}$ is a $(k, r)$-coloring of $G_{i}$ and $\left|N_{G_{i}}(x)-y\right| \geq r-1$ for $i \in\{1,2\}, c\left(N_{G_{1}}(x)-y\right)=c\left(N_{G_{2}}(x)-y\right)$ and $|D c(x)|=r-1$. Since $k \geq r+3,|A c(x)| \geq 2$. So $\left|c\left(N_{G}(y)\right)\right|=r+1$. It is a contradiction.

(ii) When $r \geq 3, k \geq 2 r+1$. Thus, $|A c(x)| \geq r$. So $\left|c\left(N_{G}(y)\right)\right| \geq r+1$. It is a contradiction.

Theorem 2.2.5. (Hall [45]) Let $G$ be a graph without $K_{3,3}$ minor. One of the followings must hold:

(i) $G$ is a planar graph;

(ii) $G \cong K_{5}$, or

(iii) $G$ can be constructed recursively by the $i$-sum of planar graphs and copies of $K_{5}$ where $i \in\{1,2\}$.

Theorem 2.2.6. Let $k$ and $r$ be integers with $r \geq 2$ and $k \geq 2 r+1$. If for any planar graph $H, \chi_{r}(H) \leq k$, then for any $K_{3,3}$-minor free graph $G, \chi_{r}(G) \leq k$.

Proof. We argue by induction on $n=|V(G)|$. If $G$ is a planar graph, then by assumption, $\chi_{r}(G) \leq k$. If $G \cong K_{5}$, then as $r \geq 2$, and so $k \geq 2 r+1 \geq 5, \chi_{r}(G) \leq k$. Assume that the 
Theorem holds for smaller value of $n$. By Theorem 2.2.5, $G=G_{1} \oplus_{i} G_{2}$, where $i \in\{1,2\}, G_{1}$ is a $K_{3,3}$-minor free graph and $G_{2}$ is either planar or is a $K_{5}$. If $G=G_{1} \oplus_{1} G_{2}$, by Proposition 2.2 .2 and $k \geq 2 r+1 \geq r+1, \chi_{r}(G) \leq \max \left\{\chi_{r}\left(G_{1}\right), \chi_{r}\left(G_{2}\right), r+1\right\} \leq k$. If $G=G_{1} \oplus_{2} G_{2}$, by Propositions 2.2.3 and 2.2.4, $\chi_{r}(G) \leq \max \left\{\chi_{r}\left(G_{1}\right), \chi_{r}\left(G_{2}\right), 2 r-1, r+3,2 r+1\right\} \leq k$ as $r \geq 2$. Thus, the Theorem 2.2.6 is proved by induction.

The following corollary follows immediately from Theorem 1.2.3 and Theorem 2.2.6.

Corollary 2.2.7. Let $G$ be a connected graph without $K_{3,3}$ minor. Then $\chi_{2}(G) \leq 5$.

Theorem 2.2.8. ( Loeb et al. [69]) If $G$ is a planar graph, then $\chi_{3}(G) \leq 10$.

Corollary 2.2.9. Let $G$ be a connected graph without $K_{3,3}$ minor. Then $\chi_{3}(G) \leq 10$.

Proof. By Theorem 2.2.8 and Theorem 2.2.6, $\chi_{3}(G) \leq 10$.

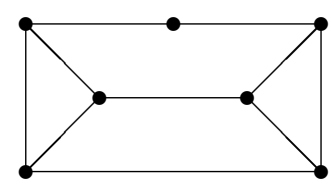

Figure A: A graph $G$ with $\chi_{3}(G)=7$

The graph presented in Figure A shows that $\chi_{3}(G)=7$ for a planar graph $G$. To the best of our knowledge, it is not known whether the upper bound of Corollary 2.2.9 is best possible or not.

\subsection{Dynamic Chromatic Number of Graphs without $K_{4}$-minors}

Definition 2.3.1. Let $G$ be a graph, $G=G_{1} \cup G_{2}$ where $G_{1}$ and $G_{2}$ are two subgraphs of $G$ such that $G_{1} \cap G_{2}=P_{k}$. We call $G$ the $k$-arc-sum of $G_{1}$ and $G_{2}$.

Theorem 2.3.2. (Lai [54]) Let $G$ be a nontrivial 2-edge-connected graph without $K_{4}$ minor. Then either $G$ is a cycle or $G$ is a proper $k$-arc-sum of some graphs $G_{1}$ and $G_{2}$, for some $k \geq 0$, with $\kappa^{\prime}\left(G_{i}\right) \geq 2,(1 \leq i \leq 2)$.

Proposition 2.3.3. (Lai et al. [57]) Let $n \geq 3$ be an integer and $C_{n}$ denote a cycle of $n$ vertices. Then

$$
\chi_{2}\left(C_{n}\right)= \begin{cases}5, & \text { if } n=5 ; \\ 3, & \text { if } n \equiv 0(\bmod 3) ; \\ 4, & \text { otherwise. }\end{cases}
$$


Proposition 2.3.4. Let $G$ be a connected graph. If $G$ contains a vertex $v$ with $d_{G}(v)=1$, then $\chi_{2}(G) \leq \chi_{2}(G-v)$ expect for $G=P_{1}, G=P_{2}$, or $G-v \cong C_{5}$. If $G-v \cong C_{5}$, then $\chi_{2}(G)=4$.

Proof. Let $G_{1}=G-v, G_{2}=K_{2}$, then $G=G_{1} \oplus_{1} G_{2}$. By Proposition 2.2.2 and $\chi_{2}\left(K_{2}\right)=2$, then $\chi_{2}(G) \leq \max \left\{\chi_{2}\left(G_{1}\right), \chi_{2}\left(G_{2}\right), 3\right\}=\chi_{2}(G-v)$ when $\chi_{2}(G-v) \geq 3$. It is easy to check that $\chi_{2}(G)=4$ when $G-v \cong C_{5}$.

Proposition 2.3.5. Let $P=v_{0} v_{1} \ldots v_{k}$ be a path of length $k>0$ and $\alpha \in\{3,4\}$. Then there exists $a(4,2)$-coloring $c$ of $P$ such that $c\left(v_{0}\right)=1, c\left(v_{k}\right)=2$ and $c\left(v_{1}\right)=\alpha$.

Proof. We will consider three cases for $k$, which are $k \equiv 0(\bmod 3), k \equiv 1(\bmod 3)$ and $k \equiv 2$ $(\bmod 3)$.

Case 1. $k \equiv 0(\bmod 3)$.

Define $c$ be a coloring such that

$$
c\left(v_{i}\right)= \begin{cases}1, & \text { if } i=0 \\ \alpha, & \text { if } i=1 \\ \{3,4\}-\alpha, & \text { if } i=2 \\ 2, & \text { if } i \bmod 3=0 \text { and } i \geq 3 \\ \alpha, & \text { if } i \bmod 3=1 \text { and } i \geq 3 \\ 1, & \text { if } i \bmod 3=2 \text { and } i \geq 3 .\end{cases}
$$

Then $c$ is a $(4,2)$-coloring of $P$ with $c\left(v_{0}\right)=1, c\left(v_{k}\right)=2$ and $c\left(v_{1}\right)=\alpha$.

Case 2. $k \equiv 1(\bmod 3)$.

If $k=1$, we can color $v_{0}$ with 1 and $v_{1}$ with $\alpha$. When $k \geq 4$, define $c$ be a coloring such that

$$
c\left(v_{i}\right)= \begin{cases}1, & \text { if } i \bmod 3=0 \\ 2, & \text { if } i \bmod 3=1 \text { and } i \geq 2 \\ \alpha, & \text { if } i=1 \\ \{3,4\}-\alpha, & \text { otherwise }\end{cases}
$$

Then $c$ is a $(4,2)$-coloring of $P$ with $c\left(v_{0}\right)=1, c\left(v_{k}\right)=2$ and $c\left(v_{1}\right)=\alpha$.

Case $3 . k \equiv 2(\bmod 3)$.

Define $c$ be a coloring such that

$$
c\left(v_{i}\right)= \begin{cases}1, & \text { if } i \quad \bmod 3=0 \\ \alpha, & \text { if } i \quad \bmod 3=1 \\ 2, & \text { otherwise }\end{cases}
$$

Then $c$ is a $(4,2)$-coloring of $P$ with $c\left(v_{0}\right)=1, c\left(v_{k}\right)=2$ and $c\left(v_{1}\right)=\alpha$.

Theorem 2.3.6. Let $G$ be a $K_{4}$-minor free graph, then $\chi_{2}(G) \leq 4$ except for $G \cong C_{5}$. 
Proof. By induction, assume that the Theorem holds for $|V(G)| \leq 4$. Assume it holds for $|V(G)|<n(n \geq 5)$. Let $G$ be a graph with $|V(G)|=n$ and $G \neq C_{5}$. W.l.o.g., assume $G$ is connected. If $G$ has a cut vertex, this theorem holds by Proposition 2.2.2. Thus we assume $G$ is 2-connected. If $G$ is a cycle, the theorem holds by Proposition 2.3.3.

By Theorem 2.3.2, $G$ is a proper $k$-arc-sum of some graphs $G_{1}$ and $G_{2}$, for some $k \geq 1$, with $\kappa^{\prime}\left(G_{i}\right) \geq 2,(1 \leq i \leq 2)$. Let $P_{i}$ be the corresponding path in $G_{i}$ with endpoints $x_{i}, y_{i}$ and $\left|E\left(P_{i}\right)\right|=k$. Let $G_{i}^{\prime}$ be the graph obtained from $G_{i}$ by shrinking $P_{i}$ into a single edge $x_{i} y_{i} \cdot(i=1,2)$. Denote $G^{\prime}=G_{1}^{\prime} \oplus_{2} G_{2}^{\prime}$ with $x y \in E\left(G^{\prime}\right)$, where $x(y)$ is obtained by identifying $x_{1}\left(y_{1}\right)$ and $x_{2}\left(y_{2}\right)$. Thus, $\chi_{2}\left(G^{\prime}\right) \leq 4$ by Proposition 2.2 .3 and induction hypothesis. Let $c^{\prime}$ be a $(4,2)$-coloring of $G^{\prime}$. W.l.o.g., assume $c^{\prime}(x)=1$ and $c^{\prime}(y)=2$. Notice that $G$ can be obtained from $G^{\prime}$ by replacing $x y$ with $P_{1}$. Let $P_{1}=v_{0} v_{1} \ldots v_{k}$ with $v_{0}=x$ and $v_{k}=y$. We assume $k \geq 2$ since $k=1$ is trivial. We are to extend $c^{\prime}$ to $V\left(P_{1}\right)-\{x, y\}$ in order to get a $(4,2)$-coloring $c$ of $G$.

Case 1. $N_{G}(x)-v_{1}$ receives at least 2 colors under coloring $c^{\prime}$ and so does for $N_{G}(y)-v_{k-1}$.

By Proposition 2.3.5, $c^{\prime}$ can be extended to $V\left(P_{1}\right)-\{x, y\}$ such that each vertex of $G$ satisfies the hued condition $(C 2)$.

Case 2. Only one of $N_{G}(x)-v_{1}$ and $N_{G}(y)-v_{k-1}$ receives one color under coloring $c^{\prime}$.

W.l.o.g., assume $c\left(N_{G}(x)-v_{1}\right)=\{\alpha\}$. Color $v_{1}$ with $\gamma^{\prime} \in\{3,4\}-\{\alpha\}$. By Proposition 2.3.5, we can extend $c$ to be a $(4,2)$-coloring of $G$.

Case 3. Both $N_{G}(x)-v_{1}$ and $N_{G}(y)-v_{k-1}$ receive one color under coloring $c^{\prime}$.

Let $c^{\prime}\left(N_{G}(x)-v_{1}\right)=\alpha$ and $c\left(N_{G}(y)-v_{k-1}\right)=\beta$. Then $\alpha, \beta \notin\{1,2\}$. In $G_{2}^{\prime}$, permute the color $\alpha$ and $\gamma$ where $\gamma \notin\{1,2, \alpha\}$ when $\alpha=\beta$; Permute the color $\alpha$ and $\beta$ in $G_{2}^{\prime}$ when $\alpha \neq \beta$. So the resulting coloring of $G^{\prime}$ is a partial $(4,2)$-coloring of $G$ where every vertex of $V(G)-\left\{v_{1}, \ldots, v_{k-1}\right\}$ satisfies the hued condition $(C 2)$. By Proposition 2.3.5, we can extend $c$ to be a $(4,2)$-coloring of $G$. 


\section{Chapter 3}

\section{On $r$-hued Coloring of Graphs without Short Induced Paths}

\subsection{Main Results}

We study the dependency of $\chi_{r}(G)$ and $\chi(G)$ among $P_{4}$-free graphs and $P_{5}$-free graphs. The main results are the following.

Theorem 3.1.1. Let $G$ be a connected $P_{4}$-free graph. Each of the following holds.

(i) $\chi_{r}(G) \leq \chi(G)+2(r-1)$, and

(ii) $\chi_{r}(G)=\chi(G)+2(r-1)$ if and only if $G=K_{s, t}$ for some integers $s \geq r$ and $t \geq r$.

Theorem 3.1.2. Let $r \geq 2$ be integers and $G$ be a connected $P_{5}$-free bipartite graph. Then $\chi_{r}(G) \leq r \chi(G)$.

Theorem 3.1.3. Let $G$ be a connected $P_{5}$-free graph. Then $\chi_{2}(G) \leq 2 \chi(G)$.

Theorems 3.1.2 and 3.1.3 are best possible in the sense that there exists infinitely many $P_{5}$-free bipartite graphs reaching the bounds. In fact, For any integers $m \geq n \geq r \geq 2$, the complete bipartite graph $K_{m, n}$ is $P_{5}$-free and by Theorem 2.3 of [57] (see Lemma 3.2.3 in Section 3.2 below $), \chi_{r}\left(K_{m, n}\right)=r \chi\left(K_{m, n}\right)$. Theorem 3.1.1 will be proved in Section 3.2, and Theorems 3.1 .2 and 3.1 .3 will be justified in the last section.

\subsection{On $r$-hued Colorings of $P_{4}$-free Graphs}

We start with a few more notations and terms to be used in this section. If $G$ is a simple graph, then $\bar{G}$ denote the complement of $G$. Let $A$ and $B$ be disjoint nonempty vertex sets. We use $K(A, B)$ to denote a complete bipartite graph with vertex bipartition $A$ and $B$. Thus 
$K(A, B) \cong K_{|A|,|B|}$. Now we assume that $A, B$ are two disjoint vertex subsets of a graph $G$, Following [11], we define $E[A, B]=\{u v \in E(G) \mid u \in A$ and $v \in B\}$. If $|E[A, B]|=|A||B|$, we say that $A$ is complete to $B$; if $E[A, B]=\emptyset$, then we say that $A$ is anti-complete to $B$; in any other cases, we say $A$ is mixed to $B$.

A graph $G$ is perfect if for any induced subgraph $H$ of $G, \chi(H)=\omega(H)$. The famous Strong Perfect Graph Theorem characterizes all perfect graphs.

Theorem 3.2.1. (Chudnovsky, Robertson, Seymour and Thomas [21]) A graph is perfect if and only if it contains no $C_{k}$ nor $\overline{C_{k}}$ as an induced subgraph, for any odd integer $k \geq 5$.

To proceed our proof, we display some properties of $P_{4}$-free graphs. As when $k \geq 5$ is an odd integer, every $C_{k}$ and every $\overline{C_{k}}$ contains an induced $P_{4}$. It follows from the Strong Perfect Graph Theorem that

$$
\text { every } P_{4} \text {-free graph must be perfect. }
$$

Next, it is known that $k$-coloring problem of $P_{4}$-free graphs can be solved in polynomial time since a $P_{4}$-free graph has a special structural property, as stated below.

Theorem 3.2.2. (Seinsche, Auxiliary Theorem of [84]) Let $G$ be a $P_{4}$-free graph, then $V(G)$ can be divided into two disjoint subsets $A, B$ such that either $A$ is complete to $B$ or $A$ is anti-complete to $B$.

By Theorem 3.2.2, it follows that if $G$ is a connected $P_{4}$-free graph, then $V(G)$ can be divided into two disjoint subsets $A$ and $B$ such that $A$ is complete to $B$. Then we have

$$
\chi(G)=\chi(G[A])+\chi(G[B]) .
$$

By $(3.1), \chi(G)=\omega(G)$. However, if $G=K(A, B)$, then for any $r \geq 2$, we have $\chi_{r}(G[A])=$ $\chi_{r}(G[B])=1$. We also note that

Lemma 3.2.3. (Lai et al. , Theorem 2.3 of [57]) For any integer $r \geq 1$, we have $\chi_{r}\left(K_{s, t}\right)=$ $\min \{2 r, s+t, r+s, r+t\}$.

Thus when $r \geq 2$, the approach mentioned in (3.2) is not applicable as $\chi_{r}(G)$ and $\chi_{r}(G[A])+$ $\chi_{r}(G[B])$ may be different. Following [11], a subset $S \subseteq V(G)$ is a stable set if $E(G[S])=\emptyset$.

Lemma 3.2.4. Let $H$ be a nontrivial $P_{4}$-free graph with $\omega(H)=k$. Let $\mathcal{F}(H)=\left\{L_{1}, L_{2}, \ldots, L_{\ell}\right\}$ be the collection of all maximum cliques of $H$. Then $H$ has a stable set $S \subseteq V(G)$ such that for any $L_{i} \in \mathcal{F}(H), V\left(L_{i}\right) \cap S \neq \emptyset, i \in\{1,2, \ldots, \ell\}$.

Proof. It suffices to prove Lemma 3.2.4 for each connected component of $H$ which contains at least one maximum clique of $H$. Hence we assume that such $H$ is connected. Let $k=1$, then the set $S=V(H)$. Therefore we assume that $k \geq 2$. Let $S$ be a subset of $V(H)$ such that

$$
E(H[S])=\emptyset \text { and } \mid\left\{i: 1 \leq i \leq \ell \text { and } V\left(L_{i}\right) \cap S \neq \emptyset\right\} \mid \text { is maximized. }
$$


If $\left\{L_{i}: V\left(L_{i}\right) \cap S \neq \emptyset\right\}=\mathcal{F}(H)$, then the lemma is proved. Therefore, without loss of generality, we may assume that $V\left(L_{1}\right) \cap S=\emptyset$. Thus $\left|V\left(L_{1}\right)\right|=k \geq 2$. Let $V\left(L_{1}\right)=\left\{v_{1}, v_{2}, \ldots\right\}$.

By (3.3), there must be a $u_{1} \in S$ and a $v_{1} \in V\left(L_{1}\right)$ such that $u_{1} v_{1} \in E(H)$, as otherwise, $S \cup\left\{v_{1}\right\}$ is a stable set and maximality of $S$ in (3.3) is violated.

Since $L_{1}$ is a maximum clique, $V\left(L_{1}\right) \cup\left\{u_{1}\right\}$ is not a clique in $H$, and so there must be a vertex $v_{2} \in V\left(L_{1}\right)-\left\{v_{1}\right\}$ such that $u_{1} v_{2} \notin E(H)$.

Assume that for some $t \geq 2$, we have found two sequences of distinct vertices $v_{1}, v_{2}, \ldots, v_{t} \in$ $V\left(L_{1}\right)$ and $u_{1}, u_{2}, \ldots, u_{t} \in S$ satisfying each of the following:

(S1) For each $i$ with $1 \leq i \leq t-1, u_{i} v_{i} \in E(H)$.

(S2) For each $i$ with $2 \leq i \leq t, u_{i-1} v_{i} \notin E(H)$.

(S3) For each $i$ with $2 \leq i \leq t$, and for each $j$ with $1 \leq j \leq i$, we have $u_{i} v_{j} \in E(H)$.

(S4) Subject to (S1), (S2) and (S3), $t$ is maximized.

By (S3), $u_{t}$ is adjacent to each of $v_{1}, v_{2}, \ldots, v_{t}$. Since $V\left(L_{1}\right)$ is a maximum clique, $V\left(L_{1}\right) \cup\left\{u_{t}\right\}$ is not a clique. Hence there must be a $v_{t+1} \in V\left(L_{1}\right)-\left\{v_{1}, \ldots, v_{t}\right\}$ such that $u_{t} v_{t+1} \notin E(H)$. By the maximality of $S$ in (3.3), there must be a $u \in S$ such that $u v_{t+1} \in E(H)$. Assume that $u \notin\left\{u_{1}, \ldots, u_{t}\right\}$. Since $u_{t} v_{t+1} \notin E(H)$ and since $H$ is $P_{4}$-free, $\left\{u_{t}, v_{t}, v_{t+1}, u\right\}$ does not induces a $P_{4}$ and so $u v_{t} \in E(H)$. Similarly, for each $h$ with $1<h \leq t$, we have $u v_{h-1} \in E(H)$. By induction on $h$ starting from $t$ to 2 , as $\left\{u_{h-1}, v_{h-1}, v_{h}, u\right\}$ does not induces a $P_{4}$, and by (S1) and (S2), $u_{h-1} v_{h-1} \in E(H)$ and $u_{h-1} v_{h} \notin E(H)$, it follows that $u v_{h-1} \in E(H)$. Thus, $u$ is adjacent to every vertex in $v_{1}, v_{2}, \ldots, v_{t}, v_{t+1}$. Thus with $u=u_{t+1}$, this is a violation to the maximality of $t$. Therefore, we must have $u=u_{i^{\prime}} \in\left\{u_{1}, u_{2}, \ldots, u_{t-1}\right\}$. By (S2) and (S3), we have $u_{i^{\prime}} v_{i^{\prime}+1} \notin E(H)$ and $u_{t} v_{i^{\prime}+1} \in E(H)$. It follows from $u_{t} v_{t+1} \notin E(H)$ and $u_{i^{\prime}} v_{t+1} \in E(H)$ that $\left\{u_{i^{\prime}}, v_{t+1}, v_{i^{\prime}+1}, u_{t}\right\}$ induces a $P_{4}$ of $H$, contrary to the assumption that $H$ is $P_{4}$-free. This proves the lemma.

Proof of Theorem 3.1.1. Observed that by Lemma 3.2.3, if $G$ is a complete bipartite graph, then $\chi_{r}\left(K_{s, t}\right) \leq 2 r=\chi(G)+2(r-1)$, where equality holds if and only if $\min \{s, t\} \geq r$. Thus it suffices to prove that if $G$ is a connected $P_{4}$-free graph, then $\chi_{r}(G) \leq \chi(G)+2(r-1)$, where equality holds only if $G$ is a bipartite graph.

To show this, let $G$ be a nontrivial and connected $P_{4}$-free graph, let $n=|V(G)|$ and $k=\chi(G)$. By Theorem 3.2.2, $V(G)$ can be partitioned into two subsets $A$ and $B$ such that $A$ is complete to $B$, or $A$ is anti-complete to $B$. Denote $G_{1}=G[A], G_{2}=G[B], \omega_{1}=\omega\left(G_{1}\right)$ and $\omega_{2}=\omega\left(G_{2}\right)$. Since $G$ is $P_{4}$-free, both $G[A]$ and $G[B]$ are $P_{4}$-free graphs.

Let $c: V(G) \mapsto \bar{k}$ be a proper coloring of $G$. By $(3.1)$, we have $\chi\left(G_{1}\right)=\omega_{1}$ and $\chi\left(G_{2}\right)=\omega_{2}$, and so. By (3.2),

$$
\chi(G)=\chi\left(G_{1}\right)+\chi\left(G_{2}\right)=\omega_{1}+\omega_{2},|c(A)|=\omega_{1} \text { and }|c(B)|=\omega_{2} .
$$

If $\max \{|A|,|B|\} \leq r-1$, then we use $|V(G)|=|A|+|B| \leq 2(r-1)$ colors so that distinct vertices 
will be colored differently. Hence this is an $(n, r)$-coloring of $G$ with $n \leq 2(r-1)$. Hence we assume that $\max \{|A|,|B|\} \geq r$. Thus, we have either $\min \{|A|,|B|\} \geq r$, or $r>\min \{|A|,|B|\}$.

Claim 1. If $\min \{|A|,|B|\} \geq r$, then $\chi_{r}(G) \leq \chi(G)+\max \left\{\left(r-\omega_{1}\right), 0\right\}+\max \left\{\left(r-\omega_{2}\right), 0\right\}$.

Proof. If both $\omega_{1} \geq r$ and $\omega_{2} \geq r$, then as $A$ is complete to $B$, it follows from Definition 1.2.1 that any proper $k$-coloring $c$ is also a $(k, r)$-coloring of $G$. Hence in this case, we have $\chi_{r}(G)=\chi(G)$.

Case 1. $\omega_{1}<r \leq \omega_{2}$ or $\omega_{2}<r \leq \omega_{1}$.

By symmetry, we assume that $\omega_{1}<r \leq \omega_{2}$. Let $h_{1}=r-\omega_{1} \leq|A|-\omega_{1}, a_{1}, a_{2}, \ldots, a_{\omega_{1}} \in$ $A$ such that $c\left(\left\{a_{1}, a_{2}, \ldots, a_{\omega_{1}}\right\}\right)=c(A)$. Randomly choose $h_{1}$ vertices $a_{\omega_{1}+1}, \ldots, a_{\omega_{1}+h_{1}}$ from $A-\left\{a_{1}, a_{2}, \ldots, a_{\omega_{1}}\right\}$. Define $c^{\prime}: V(G) \mapsto \overline{k+h_{1}}$ by

$$
c^{\prime}(x)=\left\{\begin{array}{ll}
c(x) & \text { if } x \in\left(A-\left\{a_{\omega_{1}+1}, \ldots, a_{\omega_{1}+h_{1}}\right\}\right) \cup B \\
k+j & \text { if } x=a_{\omega_{1}+j}, \text { where } 1 \leq j \leq h_{1}
\end{array} .\right.
$$

Since $c$ is a proper $k$-coloring, $c^{\prime}$ is also a proper $\left(k+h_{1}\right)$-coloring. As $A$ is complete to $B$, for each $y \in B, A \subseteq N(y)$ and so $\left|c^{\prime}(N(y))\right| \geq\left|c^{\prime}(A)\right|=\omega_{1}+h_{1}=r$. If $x \in A$, then as $\omega_{2} \geq r$, $\left|c^{\prime}(N(x))\right| \geq\left|c^{\prime}(B)\right| \geq r$. Thus by definition, $c^{\prime}$ is a proper $\left(k+h_{1}, r\right)$-coloring of $G$, and so $\chi_{r}(G) \leq k+h_{1}=\chi(G)+\left(r-\omega_{1}\right)$. This proves that Claim 1 holds in this case.

Case 2. Both $\omega_{1}<r$ and $\omega_{2}<r$.

Let $h_{1}=r-\omega_{1}, h_{2}=r-\omega_{2}$ and $\ell_{2}=k+h_{1}+h_{2}$. Let $a_{1}, a_{2}, \ldots, a_{\omega_{1}} \in A$ such that $c\left(\left\{a_{1}, a_{2}, \ldots, a_{\omega_{1}}\right\}\right)=c(A)$, and choose $h_{1}$ vertices $a_{\omega_{1}+1}, \ldots, a_{\omega_{1}+h_{1}}$ from $A-\left\{a_{1}, a_{2}, \ldots, a_{\omega_{1}}\right\} ;$ and let $b_{1}, b_{2}, \ldots, b_{\omega_{2}} \in B$ such that $c\left(\left\{b_{1}, b_{2}, \ldots, b_{\omega_{2}}\right\}\right)=c(B)$, and choose $h_{2}$ vertices $b_{\omega_{2}+1}, \ldots, b_{\omega_{2}+h_{2}}$ from $B-\left\{b_{1}, b_{2}, \ldots, b_{\omega_{2}}\right\}$. Define $c^{\prime \prime}: V(G) \mapsto \overline{\ell_{2}}$ by

$$
c^{\prime \prime}(x)=\left\{\begin{array}{ll}
c(x) & \text { if } x \in\left(A-\left\{a_{\omega_{1}+1}, \ldots, a_{\omega_{1}+h_{1}}\right\}\right) \cup\left(B-\left\{b_{\omega_{2}+1}, \ldots, b_{\omega_{2}+h_{2}}\right\}\right) \\
k+i & \text { if } x=a_{\omega_{1}+i}, \text { where } 1 \leq i \leq h_{1} \\
k+h_{1}+j & \text { if } x=b_{\omega_{2}+j}, \text { where } 1 \leq j \leq h_{2}
\end{array} .\right.
$$

Since $c$ is a proper $k$-coloring, $c^{\prime \prime}$ is also a proper $\ell_{2}$-coloring. If $x \in A$, then $\left|c^{\prime \prime}(N(x))\right| \geq$ $\left|c^{\prime \prime}(B)\right|=\omega_{2}+h_{2}=r$. If $y \in B$, then $\left|c^{\prime \prime}(N(y))\right| \geq\left|c^{\prime \prime}(A)\right|=\omega_{1}+h_{1}=r$. Hence in this case, $c^{\prime \prime}$ is a proper $\left(\ell_{2}, r\right)$-coloring of $G$. Thus $\chi_{r}(G) \leq k+h_{1}+h_{2}=\chi(G)+\left(r-\omega_{1}\right)+\left(r-\omega_{2}\right)$. This proves that Claim 1 holds in this case as well, and completes the proof of Claim 1.

Claim 2. If $r>\min \{|A|,|B|\}$, then $\chi_{r}(G) \leq \chi(G)+2 r-3$.

Proof. Without loss of generality, we assume that $|A| \geq|B|$, and so $|B|=\min \{|A|,|B|\} \leq r-1$. We proceed by induction on $\omega(G)$. Since $G$ is nontrivial and connected, $\omega(G) \geq 2$. When $\omega(G)=2, G$ is a complete bipartite graph. By Lemma 3.2.3, $\chi_{r}(G) \leq r+|B| \leq r+r-1=$ 
$2 r-1=2+2 r-3=\chi(G)+2 r-3$. Assume that $\omega(G) \geq 3$ and Claim 2 holds for smaller value of $\omega(G)$.

Case 1. $\omega_{1} \geq 2$.

Let $\mathcal{F}\left(G_{1}\right)$ denote the collection of all maximum cliques of $G_{1}$. By Lemma 3.2.4, $G_{1}$ has a stable set $S$ such that for any $L \in \mathcal{F}\left(G_{1}\right), V(L) \cap S \neq \emptyset$. It follows that $G-S$ is also $P_{4}$-free and $\omega\left(G_{1}-S\right)=\omega_{1}-1$. If $|A-S| \geq r$, by induction, $G-S$ has a $\left(k_{1}, r\right)$-coloring $c_{1}$ with $k_{1} \leq \chi(G-S)+2 r-3$. As $A-S$ and $B$ are two disjoint subsets of $G-S$ so that $A-S$ is complete to $B, \chi(G-S)=\chi(G[A-S])+\chi(G[B])=\omega\left(G_{1}-S\right)+\omega\left(G_{2}\right)=\left(\omega_{1}-1\right)+\omega_{2}$ by $(3.2)$. Thus, $k_{1} \leq\left(\omega_{1}-1\right)+\omega_{2}+2 r-3$. Extend $c_{1}$ to a $\left(k_{1}+1, r\right)$-coloring of $G$ by coloring vertices in $S$ with a new color. Thus $\chi_{r}(G) \leq \omega_{1}+\omega_{2}+2 r-3=\chi(G)+2 r-3$. If $|A-S| \leq r-1$, then we use $|V(G-S)|=|A-S|+|B| \leq 2(r-1)$ colors so that distinct vertices will be colored differently. Let $c_{2}$ be a $\left(k_{2}, r\right)$-coloring of $G-S$ with $k_{2} \leq 2(r-1)$. Extend $c_{2}$ to a $\left(k_{2}+1, r\right)$-coloring of $G$ by coloring vertices in $S$ with a new color. Thus, as $\chi(G)=\omega(G) \geq 3$, $\chi_{r}(G) \leq 1+2(r-1)<\chi(G)+2 r-3$.

Case 2. $\omega_{2} \geq 2$.

Similarly, let $\mathcal{F}\left(G_{2}\right)$ denote the collection of all maximum cliques of $G_{2}$. By Lemma 3.2.4, $G_{2}$ has a stable set $S^{\prime}$ such that for any $L^{\prime} \in \mathcal{F}\left(G_{2}\right), V\left(L^{\prime}\right) \cap S^{\prime} \neq \emptyset$. It follows that $G-S^{\prime}$ is also $P_{4}$-free and $\omega\left(G_{2}-S^{\prime}\right)=\omega_{2}-1$. By induction, $G-S^{\prime}$ has a $\left(k_{3}, r\right)$-coloring $c_{3}$ with $k_{3} \leq \chi\left(G-S^{\prime}\right)+2 r-3$. As $A$ and $B-S^{\prime}$ are two disjoint subsets of $G-S^{\prime}$ so that $A$ is complete to $B-S^{\prime}, \chi\left(G-S^{\prime}\right)=\chi(G[A])+\chi\left(G\left[B-S^{\prime}\right]\right)=\omega\left(G_{1}\right)+\omega\left(G_{2}-S^{\prime}\right)=\omega_{1}+\left(\omega_{2}-1\right)$ by (3.2). Thus, $k_{3} \leq \omega_{1}+\left(\omega_{2}-1\right)+2 r-3$. Extend $c_{3}$ to a $\left(k_{3}+1, r\right)$-coloring of $G$ by coloring vertices in $S^{\prime}$ with a new color. Thus, $\chi_{r}(G) \leq \omega_{1}+\omega_{2}+2 r-3=\chi(G)+2 r-3$.

As both Case 1 and Case 2 lead to the conclusion of Claim 2, Claim 2 is justified by induction.

Since $\omega_{1} \geq 1$ and $\omega_{2} \geq 1$, it follows by Claims 1 and 2 that if $G$ is a $P_{4}$-free graph, then $\chi_{r}(G) \leq \chi(G)+2(r-1)$. Thus Theorem 3.1.1(i) follows. Furthermore, equality $\chi_{r}(G)=$ $\chi(G)+2(r-1)$ holds if and only if $\omega_{1}=\omega_{2}=1$, which implies that $G=K(A, B)$. This completes the proof of Theorem 3.1.1.

\subsection{On $r$-hued Colorings of $P_{5}$-free Graphs}

In this section, we investigate the relationsp between $\chi_{r}(G)$ and $\chi(G)$ for a $P_{5}$-free graph $G$. We start with some examples. 
Example 1. Let $k \geq 2$ and $r \geq 1$ be integers. There exists a family $\mathcal{F}$ of connected $P_{5}$-free graphs, such that every $G \in \mathcal{F}$ satisfies $\chi_{r}(G)=r \chi(G)$.

For convenience, in this example, we often use $\bar{k}$ for $\mathbb{Z}_{k}$, the additive group of integers modulo $k$. For positive integers $n_{1}, n_{2}, \ldots, n_{k}$, let $K=K_{n_{1}, n_{2}, \ldots, n_{k}}$ denote a complete $k$-partite graph such that the vertex parti-sets are $V_{1}, V_{2}, \ldots, V_{k}$ with $\left|V_{i}\right|=n_{i} \geq r, 1 \leq i \leq k$. Let $U=\left\{u_{1}, u_{2}, \ldots, u_{k}\right\}$ be a set of vertices with $U \cap V(K)=\emptyset$; and let $n=n_{1}+n_{2}+\ldots+n_{k}+k$. Obtain a graph $G=G(n, k)$ from $K$ and $U$ by joining $u_{i}$ to every vertex in $V_{i}$ but not to any other vertices, for each $i$ with $1 \leq i \leq k$. Thus $n=|V(K)|+|U|=|V(G)|$. Let $\mathcal{F}$ be the collection of all graphs $G(n, k, r)$ for some values $n, k, r$ with $n \geq k \geq r$ and $r \geq 1$.

Proposition 3.3.1. For any graph $G \in \mathcal{F}$, each of the following holds.

(i) $\chi(G)=\omega(G)=k$.

(ii) $\chi_{r}(G)=r k$.

(iii) $G$ is $P_{5}$-free.

Proof. Let $G \in \mathcal{F}$. Then for some integers $n$ and $k$, we have $G=G(n, k, r)$. We shall use the same notation above in our arguments. For each $i$ with $1 \leq i \leq k$, fix a vertex $w_{i} \in V_{i}$; and let $W=\left\{w_{1}, w_{2}, \ldots, w_{k}\right\}$. Since $K$ is a complete $k$-partite graph, $G[W] \cong K_{k}$.

(i) By definition of $G, G[W] \cong K_{k}$ is a clique of $G$ and so $\chi(G) \geq \omega(G)=k$. Let $c: V(G) \mapsto \bar{k}$ be so defined that $c\left(V_{i}\right)=i$ and $c\left(u_{i}\right)=i+1(\bmod k)$ Since $K$ is a $k$-partite graph, each $V_{i}$ is a stable set; since $N_{G}\left(u_{i}\right)=V_{i}$, it follows that $c$ is a proper $k$-coloring of $G$. This proves (i).

(ii) Suppose that $\ell=\chi_{r}(G)$ and let $c: V(G) \mapsto \bar{\ell}$ be a $(k, r)$-coloring of $G$. Since $G[W] \cong K_{k}$, we may assume that for each $i$ with $1 \leq i \leq k, c\left(w_{i}\right)=i$.

Fix an $i$ with $1 \leq i \leq k$. Since $n_{i} \geq r$ and $N_{G}\left(u_{i}\right)=V_{i}$, there must be a vertex subset $Z_{i} \subseteq V_{i}$ such that $\left|c\left(Z_{i}\right)\right|=\left|Z_{i}\right|=r$. Randomly pick a vertex $z_{i} \in Z_{i}$, and let $Z=\left\{z_{1}, z_{2}, \ldots, z_{k}\right\}$. As $K$ is a complete $k$-partite graph, $G[Z] \cong K_{k}$ and so $|c(Z)|=k$. It follows that $\ell \geq\left|c\left(\cup_{i=1}^{k} Z_{i}\right)\right|=r k$.

To justify (ii), it suffices to present a $(r k, r)$-coloring of $G$. Construct a mapping $c: V(G) \mapsto$ $\mathbb{Z}_{r k}$ as follows. Fix an $i$ with $i \in \mathbb{Z}_{k}$ and define $c\left(V_{i}\right)=\{(i-1) r,(i-1) r+1, \ldots,(i-1) r+r-1\} \subseteq$ $\mathbb{Z}_{r k}$. As $K$ is a complete $k$-partite graph with $k \geq r$, the restriction of $c$ to $V(K)$ is a $(r k, r)$ coloring. Since $N_{G}\left(u_{i}\right)=V_{i}$, and since $\left|c\left(V_{i}\right)\right|=r$, it follows that $c$ is indeed a $(r k, r)$-coloring. This proves that $\ell=\chi_{r}(G) \leq r k$, and so completes the proof of (ii).

(iii) Let $P=x_{1} x_{2} x_{3} \ldots x_{t}$ be a longest induced path in $G$. Since $K$ is a complete $k$-partite graph, and since $P$ is induced, we must have $|V(P) \cap V(K)| \leq 3$ and $|V(P) \cap V(K)|=3$ if and only if $V(P) \cap V(K)=\left\{x_{i-1}, x_{i}, x_{i+1}\right\}$ for some $i$ with $1<i<5$ such that $x_{i-1}$ and $x_{i+1}$ are in the same partite set of $K$. If $x_{i-1}$ and $x_{i+1}$ are both in a $V_{j}$, then we must have $t=3$ and $P=x_{i-1} x_{i} x_{i+1}$ since $N\left(u_{j}\right)=V_{j}$. If $|V(P) \cap V(K)|=2$, then as $P$ is a longest induced path, $V(P) \cap V(K)=\left\{x_{i-1}, x_{i}\right\}$. We may assume, without lot of generality, that $x_{i-1} \in V_{1}$ and $x_{i} \in V_{2}$. It follows that $P=u_{1} x_{i-1} x_{i} u_{2}$. Hence in any case, $|V(P)|=4$ and so $G$ must be 
$P_{5}$-free.

Theorem 3.1.1 indicates that $f(k, r, 4)=k+2(r-1)$, answering the problem when $t=4$ for any $r$ and $k$. In this section we will prove Theorems 3.1.2 and 3.1.3. Theorem 3.1.3 suggests $c(2,5)=2$, providing evidences for $c(r, 5)=r$.

A subgraph $H$ of $G$ is dominating if every vertex of $G$ is either in $V(H)$ or is adjacent to a vertex in $H$. A subset $V^{\prime} \subseteq V(G)$ is dominating if $G\left[V^{\prime}\right]$ is dominating. Bacso and Tuza ([8]) indicated that a $P_{5}$-free graph always contains a dominating maximal clique or a $P_{3}$.

Theorem 3.3.2. (Bacso and Tuza [8]) If $G$ is a connected $P_{5}$-free graph, then $G$ has a dominating clique or a dominating $P_{3}$.

Using Theorem 3.3.2, Hoang et al. in [47] indicated that for $P_{5}$-free graphs, the $k$-coloring problem can be solved in polynomial time. We will also apply this structural property of $P_{5}$-free graphs to investigate the relationship between $\chi_{r}(G)$ and $\chi(G)$ for a $P_{5}$-free graph $G$.

Lemma 3.3.3. Let $r$ and $s$ be integers with $r \geq 2$ and $s \geq 3, G$ be a connected graph with a dominating subgraph $K$ where $K$ is isomorphic to a $K_{s}$. Let $k=\chi_{r-1}(G-V(K))+s$. The $G$ has a $(k, 1)$-coloring $c: V(G) \mapsto \bar{k}$ such that for any vertex $v \in V(G)-V(K)$,

$$
\left|c\left(N_{G}(v)\right)\right| \geq \min \left\{d_{G}(v), r\right\}
$$

Proof. Let $k_{1}=\chi_{r-1}(G-V(K))$ and $k=k_{1}+s$. We first let $c_{1}: V(G-V(K)) \mapsto \overline{k_{1}}$ be a $\left(k_{1}, r-1\right)$-coloring of $G-V(K)$. Extend $c_{1}$ to a $(k, 1)$-coloring $c: V(G) \mapsto \bar{k}$ by coloring $V(K)$ with $s=|V(K)|$ new colors in $\left\{k_{1}+1, k_{1}+2, \ldots, k_{1}+s\right\}$.

For each vertex $v \in V(G)-V(K)$, since $c_{1}$ is a $\left(k_{1}, r-1\right)$-coloring of $G-V(K)$,

$$
\left|c_{1}\left(N_{G-V(K)}(v)\right)\right| \geq \min \left\{r-1, d_{G-V(K)}(v)\right\} .
$$

As $\left|c\left(N_{G}(v) \cap V(K)\right)\right|=\left|N_{G}(v) \cap V(K)\right|$ and $K$ is a dominating subgraph of $G$, it follows that (3.5) must hold. This proves the lemma.

Corollary 3.3.4. Let $r$ and $s$ be integers with $r \geq 2$ and $s \geq 3, G$ be a connected graph with a dominating subgraph $K$ where $K$ is isomorphic to a $K_{s}$. If $r \leq s$ and if $\chi_{r-1}(G-V(K)) \leq$ $(r-1) \chi(G)$, then $\chi_{r}(G) \leq r \chi(G)$.

Proof. By Lemma 3.3.3, $G$ has a $(k, 1)$-coloring $c: V(G) \mapsto \bar{k}$ such that for any vertex $v \in V(G)-V(K)$, (3.5) holds. Since $K$ is a complete graph on $s \geq r$ vertices, we have $\chi(G) \geq|V(K)|=s$, and every vertex $v \in V(K)$ also satisfies (3.5). Hence $c$ is a $(k, r)$-coloring of $G$, and so $\chi_{r}(G) \leq \chi_{r-1}(G-V(K))+s \leq(r-1) \chi(G)+\chi(G)$. 


\subsubsection{On $r$-hued Colorings of $P_{5}$-free Bipartite Graphs}

For a subset $S \subseteq V(G)$, define $N_{G}(S)=\cup_{v \in S} N_{G}(v)$. Recall that $K(A, B)$ to denote the complete bipartite graph with vertex bipartition $(A, B)$. We start with a few definitions and lemmas.

Definition 3.3.5. Let $P_{3}=w_{1} w_{2} w_{3}$ be a dominating path of a connected graph $G$. For $i=$ $1,2,3$, define $V_{i}=\left\{v \in V(G): v w_{i} \in E(G)\right\}$.

With the notation in Definition 3.3.5, we have the following observations, which follow from Definition 3.3.5 and from the fact that a bipartite graph contains no cycles of odd length.

Observation 1. Let $G$ is bipartite and $P_{5}$-free with $w_{1} w_{2} w_{3}$ being a dominating path. Each of the following holds.

(i) Either $V_{1} \subseteq V_{3}$ or $V_{3} \subseteq V_{1}$.

(ii) $E\left(G\left[V_{1} \cup V_{3}\right]\right)=\emptyset$, and $E\left(G\left[V_{2}\right]\right)=\emptyset$.

(iii) For any $v \in V_{1} \cup V_{3}, N_{G}(v) \subseteq V_{2}$.

(iv) For any $v \in V_{2}, N_{G}(v) \subseteq V_{1} \cup V_{3}$.

Lemma 3.3.6. Let $G$ be a connected $P_{5}$-free graph with a dominating $P_{3}=w_{1} w_{2} w_{3}$. If $G$ is bipartite, then either $V_{2}=\left\{w_{1}, w_{3}\right\}$, or $\left|V_{2}\right| \geq 3$ and for any $v \in V_{2}-\left\{w_{1}, w_{3}\right\}$, one of the followings holds.

(i) $N_{G}(v)=\left\{w_{2}\right\}$.

(ii) For any $u \in V_{1}-V_{3}$, if $u v \in E(G)$, then $V_{3} \subseteq N_{G}(v)$.

(iii) For any $u \in V_{3}-V_{1}$, if $u v \in E(G)$, then $V_{1} \subseteq N_{G}(v)$.

Proof. As $w_{1}, w_{3} \in V_{2}$, we have $\left|V_{2}\right| \geq 2$. Assume that $\left|V_{2}\right| \geq 3$ and (i) does not hold, we are to show that one of (ii) and (iii) must hold. By symmetry, it suffices to justify (ii).

Suppose that there exists a vertex $u \in V_{1}-V_{3}$ with $u v \in E(G)$. For any $u^{\prime} \in V_{3}-\left\{w_{2}\right\}$, $P=u v w_{2} w_{3} u^{\prime}$ is a path on 5 vertices in $G$. Since $G$ is bipartite, then $u w_{2}, w_{2} u^{\prime}, v w_{3}, u u^{\prime} \notin$ $E(G)$. Also $u w_{3} \notin E(G)$ and since $G$ is $P_{5}$-free, we must have $u^{\prime} v \in E(G)$. This implies that $V_{3} \subseteq N_{G}(v)$.

Lemma 3.3.7. Let $G$ be a connected $P_{5}$-free bipartite graph on $n=|V(G)|$ vertices with a dominating $P_{3}=w_{1} w_{2} w_{3}$ such that $\left|V_{2}\right| \geq 3$. Adopting the notation in Definition 3.3 .5 and defining $V_{21}=\left\{v \in V_{2}: N_{G}(v) \cap\left(V_{1}-V_{3}\right) \neq \emptyset\right\}$, each of the followings holds.

(i) If $V_{1}=V_{3}$, then for any $u, u^{\prime} \in V_{3}$, if $d_{G}\left(u^{\prime}\right) \leq d_{G}(u)$, then $N_{G}\left(u^{\prime}\right) \subseteq N_{G}(u)$; and for any $v, v^{\prime} \in V_{2}$, if $d_{G}\left(v^{\prime}\right) \leq d_{G}(v)$, then $N_{G}\left(v^{\prime}\right) \subseteq N_{G}(v)$.

(ii) If $V_{3} \subset V_{1}$ and $V_{1}-V_{3} \neq \emptyset$, then each of the following holds.

(ii-1) $G\left[V_{21} \cup V_{3}\right]=K\left(V_{21}, V_{3}\right)$ is a complete bipartite graph.

(ii-2) For any $u, u^{\prime} \in V_{1}$, if $d_{G}\left(u^{\prime}\right) \leq d_{G}(u)$, then $N_{G}\left(u^{\prime}\right) \subseteq N_{G}(u)$; for any $v, v^{\prime} \in V_{2}$, if $d_{G}\left(v^{\prime}\right) \leq d_{G}(v)$, then $N_{G}\left(v^{\prime}\right) \subseteq N_{G}(v)$. 
Proof. (i). By Observation 1 (iii), for any vertex $u \in V_{3}-\left\{w_{2}\right\}, d\left(w_{2}\right) \geq d(u)$ and $N_{G}(u) \subseteq$ $N_{G}\left(w_{2}\right)$. And by Observation 1 (iv), if $V_{1}=V_{3}$, then $d\left(w_{1}\right)=d\left(w_{3}\right) \geq d(v)$ and $N_{G}(v) \subseteq$ $N_{G}\left(w_{1}\right)=N_{G}\left(w_{3}\right)=V_{3}$ for any vertex $v \in V_{2} \backslash\left\{w_{1}, w_{3}\right\}$.

Suppose that $u, u^{\prime} \in V_{3} \backslash\left\{w_{2}\right\}$ with $d_{G}(u) \geq d_{G}\left(u^{\prime}\right)$. By contradiction, we assume that $N_{G}\left(u^{\prime}\right)-N_{G}(u) \neq \emptyset$. Since $d_{G}(u) \geq d_{G}\left(u^{\prime}\right)$ and $N_{G}\left(u^{\prime}\right)-N_{G}(u) \neq \emptyset$, we also have $N_{G}(u)-$ $N_{G}\left(u^{\prime}\right) \neq \emptyset$. Pick a vertex $v^{\prime} \in N_{G}\left(u^{\prime}\right)-N_{G}(u)$ and a vertex $v \in N_{G}(u)-N_{G}\left(u^{\prime}\right)$, where $v, v^{\prime} \in V_{2} \backslash\left\{w_{1}, w_{3}\right\}$. Then $P=u v w_{2} v^{\prime} u^{\prime}$ is a path on 5 vertices in $G$. Since $G$ is bipartite, $u w_{2}, u u^{\prime}, w_{2} u^{\prime} \notin E(G)$. Since $G$ is $P_{5}$-free, one of $u v^{\prime}, u^{\prime} v$ must be in $E(G)$, contrary to the assumptions that $v^{\prime} \in N_{G}\left(u^{\prime}\right)-N_{G}(u)$ and $v \in N_{G}(u)-N_{G}\left(u^{\prime}\right)$. Hence we must have $N_{G}\left(u^{\prime}\right) \subseteq$ $N_{G}(u)$.

Similarly, assume that there exist vertices $v, v^{\prime} \in V_{2} \backslash\left\{w_{1}, w_{3}\right\}$ with $d_{G}(v) \geq d_{G}\left(v^{\prime}\right)$ and $N_{G}\left(v^{\prime}\right)-N_{G}(v) \neq \emptyset$. Since $d_{G}(v) \geq d_{G}\left(v^{\prime}\right)$ and $N_{G}\left(v^{\prime}\right)-N_{G}(v) \neq \emptyset$, we also have $N_{G}(v)-$ $N_{G}\left(v^{\prime}\right) \neq \emptyset$. Pick a vertex $u^{\prime} \in N_{G}\left(v^{\prime}\right)-N_{G}(v)$ and a vertex $u \in N_{G}(v)-N_{G}\left(v^{\prime}\right)$, where $u, u^{\prime} \in V_{3}$. Thus $Q=u v w_{2} v^{\prime} u^{\prime}$ is a path on 5 vertices in $G$. Since $G$ is bipartite, $u w_{2}, v v^{\prime}, w_{2} u^{\prime} \notin$ $E(G)$. Since $G$ is $P_{5}$-free, one of $u v^{\prime}, u^{\prime} v$ must be in $E(G)$, contrary to the assumptions that $u \in N_{G}(v)-N_{G}\left(v^{\prime}\right)$ and $u^{\prime} \in N_{G}\left(v^{\prime}\right)-N_{G}(v)$. This completes the proof of (i).

(ii). Suppose that $V_{3} \subset V_{1}$ and $V_{1}-V_{3} \neq \emptyset$. By Lemma 3.3.6(ii), for any $v \in V_{21}, V_{3} \subseteq N_{G}(v)$. Hence $G\left[V_{21} \cup V_{3}\right]=K\left(V_{21}, V_{3}\right)$, and so (ii-1) follows.

By Observation 1 (iv), if $V_{3} \subset V_{1}$, then $d\left(w_{1}\right) \geq d(v)$ and $N_{G}(v) \subseteq N_{G}\left(w_{1}\right)=V_{1}$ for any $v \in V_{2} \backslash\left\{w_{1}\right\}$. If $v \in V_{21}$, then by (ii-1), we have $N_{G}\left(w_{3}\right)=V_{3} \subset N_{G}(v)$. If $v \in V_{2} \backslash V_{21}$, then $N_{G}(v) \subset V_{3}=N_{G}\left(w_{3}\right)$. Suppose $v, v^{\prime} \in V_{2} \backslash\left\{w_{1}, w_{3}\right\}$ with $d_{G}(v) \geq d_{G}\left(v^{\prime}\right)$, the proof for $N_{G}\left(v^{\prime}\right) \subseteq N_{G}(v)$ is similar to that for (i), so it will be omitted. As for any two vertices $u, u^{\prime} \in V_{1}$ with $d_{G}(u) \geq d_{G}\left(u^{\prime}\right)$, the proof for $N_{G}\left(u^{\prime}\right) \subseteq N_{G}(u)$ is also similar to that for (i). Thus (ii-2) is justified.

Lemma 3.3.8. Let $G$ be a bipartite $P_{5}$-free graph with a vertex bipartition $(U, V)$. If $G$ has a dominating $P_{3}$, then $G$ has a $(2 r, r)$-coloring $c: V(G) \mapsto \overline{2 r}$ in such a way that $c(U) \subseteq \bar{r}$ and $c(V) \subseteq \overline{2 r}-\bar{r}$. In particular, $\chi_{r}(G) \leq 2 r$.

Proof. It suffices to prove Lemma 3.3.8 for connected graphs. Hence we assume that $G$ is a connected bipartite $P_{5}$-free graph with a dominating $P_{3}$. Let $V\left(P_{3}\right)=\left\{w_{1}, w_{2}, w_{3}\right\}$ and define $V_{i}=\left\{v \in V(G) \mid v w_{i} \in E(G)\right\}$, for $i=1,2,3$ as in Definition 3.3.5. Set $U=V_{1} \cup V_{3}$ and $V=V_{2}$. By Observation 1 (ii) - (iv), $G$ is a bipartite graph with $(U, V)$ being its vertex bipartition. By Lemma 3.3.6, either $V_{2}=\left\{w_{1}, w_{3}\right\}$, or $\left|V_{2}\right| \geq 3$ and for any $v \in V_{2}-\left\{w_{1}, w_{3}\right\}$, one of the Lemma 3.3.6 (i), (ii) and (iii) must hold.

Assume first that $V_{2}=\left\{w_{1}, w_{3}\right\}$. Then $V(G)=\left\{w_{1}, w_{3}\right\} \cup V_{1} \cup V_{3}$. Without loss of generality, we may assume $V_{3} \subseteq V_{1}$. Then $G$ is a bipartite graph with partite sets $\left\{w_{1}, w_{3}\right\}$ and $V_{1}$. Let $c: V(G) \mapsto \overline{r+2}$ be a $(r+2,1)$-coloring of $G$ so that $c\left(V_{1}\right) \subseteq \bar{r}$ with $\left|c\left(V_{i}\right)\right|=\min \left\{\left|V_{i}\right|, r\right\}$ for 
$i \in\{1,3\}$ and $c\left(V_{2}\right)=c\left(\left\{w_{1}, w_{3}\right\}\right)=\{r+1, r+2\}$. Thus Lemma 3.3.8 holds.

Next we assume that $\left|V_{2}\right| \geq 3$. In the rest of the proof, we shall adopt the notation in Definition 3.3.5 and in Lemma 3.3.7. By Observation 1 (i) and by symmetry, we may assume either $V_{1}=V_{3}$ or $V_{3} \subset V_{1}, V_{1} \backslash V_{3} \neq \emptyset$.

Denote $V_{1}=\left\{u_{1}, u_{2}, \ldots, u_{h}\right\}$ and $V_{2}=\left\{v_{1}, v_{2}, \ldots, v_{\ell}\right\}$ such that

$$
d_{G}\left(u_{1}\right) \geq d_{G}\left(u_{2}\right) \geq \ldots \geq d_{G}\left(u_{h}\right), \text { and } d_{G}\left(v_{1}\right) \geq d_{G}\left(v_{2}\right) \geq \ldots \geq d_{G}\left(v_{\ell}\right) .
$$

Then by Lemma 3.3.7 (i) and (ii), we have

$$
V_{1} \supseteq N_{G}\left(v_{1}\right) \supseteq N_{G}\left(v_{2}\right) \supseteq \ldots \supseteq N_{G}\left(v_{\ell}\right), \text { and } V_{2} \supseteq N_{G}\left(u_{1}\right) \supseteq N_{G}\left(u_{2}\right) \supseteq \ldots \supseteq N_{G}\left(u_{h}\right) \text {. }
$$

By (3.6), it is possible to relabel $V_{1}=\left\{x_{1}, x_{2}, \ldots, x_{h}\right\}$ so that for each $i$ with $1 \leq i \leq \ell$, there exists a subscript $n_{i} \leq h$ such that $N_{G}\left(v_{i}\right)=\left\{x_{1}, x_{2}, \ldots, x_{n_{i}}\right\}$. Similarly, we can relabel $V_{2}=\left\{y_{1}, y_{2}, \ldots, y_{\ell}\right\}$ so that for each $j$ with $1 \leq j \leq h$, there exists a subscript $k_{j} \leq \ell$ such that $N_{G}\left(u_{j}\right)=\left\{y_{1}, y_{2}, \ldots, y_{k_{j}}\right\}$. Define $c: V(G) \mapsto \overline{2 r}$ to be a mapping satisfying the following.

(M1) For $i=1,2, \ldots, h$, choose $j=j(i)$ with $1 \leq j \leq r$ and $i \equiv j(\bmod r)$, and define $c\left(x_{i}\right)=j$. Thus $c\left(V_{1}\right) \subseteq \bar{r}$ and $\left|c\left(V_{1}\right)\right| \geq \min \left\{\left|V_{1}\right|, r\right\}$.

(M2) For $i=1,2, \ldots, \ell$, choose $j=j(i)$ with $r+1 \leq j \leq 2 r$ and $i \equiv j(\bmod r)$, and define $c\left(y_{i}\right)=j$. Thus $c\left(V_{2}\right) \subseteq \overline{2 r}-\bar{r}$ and $\left|c\left(V_{2}\right)\right| \geq \min \left\{\left|V_{2}\right|, r\right\}$.

To see that $c$ is a $(2 r, 1)$-coloring of $G$, we take any edge $x y \in E(G)$. Since $G$ is a bipartite graph with vertex bipartition $\left(V_{1}, V_{2}\right)$, we may assume that $x \in V_{1}$ and $y \in V_{2}$. Then by (M1) and (M2), we have $c(x) \neq c(y)$ and so $c$ is a $(2 r, 1)$-coloring of $G$. To see that $c$ is indeed a $(2 r, r)$-coloring of $G$, we pick an arbitrary vertex $z \in V(G)$. If $z \in V_{1}$, then $z=x_{i}$ for some $i$ with $1 \leq i \leq h$. By (M1), either $n_{i} \leq r$ and $\left|c\left(N_{G}\left(x_{i}\right)\right)\right|=\left|N_{G}\left(x_{i}\right)\right|$, or $n_{i} \geq r$ and $\left|c\left(N_{G}\left(x_{i}\right)\right)\right| \geq r$. Similarly, if $z \in V_{2}$, then using (M2), we also conclude that $\left|c\left(N_{G}(z)\right)\right| \geq \min \left\{\left|N_{G}(z)\right|, r\right\}$. Thus $c$ is a $(2 r, r)$-coloring of $G$ satisfying $c(U) \subseteq \bar{r}$ and $c(V) \subseteq \overline{2 r}-\bar{r}$. This completes the proof of Lemma 3.3.8.

Lemma 3.3.9. Let $G$ be a connected $P_{5}$-free bipartite graph. If $G$ has a dominating $K_{2}$, then $\chi_{r}(G) \leq 2 r$.

Proof. Throughout the proof of this lemma, let $(U, V)$ denote the vertex bipartition of $G$. We shall prove the lemma arguing by induction on $r$. Since $G$ is bipartite, Lemma 3.3.9 holds for $r=1$. We assume that $r>1$ and that Lemma 3.3.9 holds for smaller values of $r$.

Since $G$ has a dominating $K_{2}$, there exist a pair of adjacent vertices $u_{0}, v_{0}$ such that $V(G)=N_{G}\left(u_{0}\right) \cup N_{G}\left(v_{0}\right)$ and such that $N_{G}\left(u_{0}\right)=V$ and $N_{G}\left(v_{0}\right)=U$. Define $G^{\prime}=G-\left\{u_{0}, v_{0}\right\}$. Then $G^{\prime}$ is also a $P_{5}$-free bipartite graph. Let $H_{1}, H_{2}, \ldots, H_{t}$ be the connected components of $G^{\prime}$. Then each $H_{i}$ is a connected $P_{5}$-free bipartite graph. By Theorem 3.3.2 and since $H_{i}$ is bipartite, if $\left|E\left(H_{i}\right)\right|>0$, then $H_{i}$ has a dominating $P_{2}$ or a dominating $P_{3}$. Thus by induction 
and by Lemma 3.3.8, $G^{\prime}$ has a $(2 r-2, r-1)$-coloring $c^{\prime}: V\left(G^{\prime}\right) \mapsto \overline{2 r-2}$ satisfying the following properties:

(R1) For each $i$ with $1 \leq i \leq t$, if $\left|E\left(H_{i}\right)\right|>0$, then $c^{\prime}\left(U \cap V\left(H_{i}\right)\right) \subseteq \overline{r-1}$ and $c^{\prime}\left(V \cap V\left(H_{i}\right)\right) \subseteq$ $\overline{2 r-2}-\overline{r-1}$.

(R2) Both $\left|c^{\prime}(U)\right| \geq \min \left\{\left|U-\left\{u_{0}\right\}\right|, r-1\right\}$ and $\left|c^{\prime}(V)\right| \geq \min \left\{\left|V-\left\{v_{0}\right\}\right|, r-1\right\}$.

We extend $c^{\prime}$ to $c: V(G) \mapsto \overline{2 r}$ as follows:

$$
c(v)= \begin{cases}c^{\prime}(v) & \text { if } v \in V\left(G^{\prime}\right)=V(G)-\left\{u_{0}, v_{0}\right\} \\ 2 r-1 & \text { if } v=u_{0} \\ 2 r & \text { if } v=v_{0} .\end{cases}
$$

Since $c^{\prime}$ is a $(2 r-2, r-1)$-coloring of $G^{\prime}$ satisfying (R1) and (R2), and since $N_{G}\left(u_{0}\right)=V$ and $N_{G}\left(v_{0}\right)=U$, it follows by the definition of $c$ that $c$ is a $(2 r, r)$-coloring of $G$.

Theorem 3.3.10. If $G$ is a bipartite $P_{5}$-free graph. Then for any $r \geq 2$,

$$
\chi_{r}(G) \leq 2 r
$$

Proof. By Theorem 3.3.2 and since $G$ is bipartite, $G$ has a dominating path $P_{t}$ with $t=2$ or 3. Therefore, Theorem 3.3.10 follows from Lemmas 3.3.8 and 3.3.9.

\subsubsection{On 2-hued Colorings of $P_{5}$-free Graphs}

In this section, we shall prove Theorem 3.1.3.

Proof of Theorem 3.1.3. It suffices to prove Theorem 3.1.3 for connected $P_{5}$-free graphs. By Theorem 3.3.2, $G$ has a dominating clique $K_{s}$ for some $s \geq 1$ or a dominating $P_{3}$. Let $J$ be a dominating maximal clique $K_{s}$ or a dominating $P_{3}$ of $G$. By Theorem 3.3.10, we may assume that $G$ is not bipartite. If $J=K_{1}$, then $E(G)=\emptyset$ and $G=K_{1}$, and so nothing needs to be proved. If $J=K_{s}$ for some $s \geq 3$, then by Corollary 3.3.4, we have $\chi_{2}(G) \leq 2 \chi(G)$. Hence we assume that $J \in\left\{K_{2}, P_{3}\right\}$.

Let $k=\chi(G)$ and let $c_{1}: V(G) \mapsto \bar{k}$ be a $(k, 1)$-coloring of $G$. We also use $c_{1}: V(G)-V(J) \mapsto$ $\bar{k}$ be the restriction of $c_{1}$. Let $|V(J)|=\ell$ and $V(J)=\left\{w_{1}, w_{2}, \ldots, w_{\ell}\right\}$. Define $c: V(G) \mapsto \overline{k+\ell}$ as follows.

$$
c(v)= \begin{cases}c_{1}(v) & \text { if } v \in V(G)-V(J) \\ k+j & \text { if } v=w_{j} \in V(J), \quad 1 \leq j \leq \ell .\end{cases}
$$

Since $c_{1}$ is a $(k, 1)$-coloring of $G$, we conclude that $c$ is also a $(k, 1)$-coloring of $G$. By the definition of a dominating subgraph, if $v \in V(G)-V(J)$, then either $v$ is of degree one in $G$, or $v$ is adjacent to at least one vertex in $V(G)-V(J)$, or $v$ is adjacent to at least two vertices of $V(J)$. In any case, $\left|c\left(N_{G}(v)\right)\right| \geq \min \left\{d_{G}(v), 2\right\}$. Similarly, for any $v \in V(J)$, we also 
have $\left|c\left(N_{G}(v)\right)\right| \geq \min \left\{d_{G}(v), 2\right\}$. It follows by Definition 1.2.1 that $c$ is a $(k+\ell, 2)$-coloring of $G$, and so $\chi_{2}(G) \leq \chi(G)+\ell$. Since $G$ is not bipartite, we have $\chi(G) \geq 3 \geq \ell$, and so $\chi_{2}(G) \leq \chi(G)+\ell \leq 2 \chi(G)$.

\subsection{Future Studies}

Proposition 3.3.1 leads to the following Problem.

Problem 1. For integers $k>0, r \geq 2$ and $t \geq 4$, determine a best possible function $f(k, r, t)$ such that for every connected $P_{t}$-free graph $G$ with $\chi(G)=k$, we have $\chi_{r}(G) \leq f(k, r, t)$. More specifically, is there a best possible value $c=c(r, t)$ such that for every connected $P_{t}$-free graph $G$, we have $\chi_{r}(G) \leq c(r, t) \chi(G)$ ? In particular, can $c(r, 5)=r$ ? 


\section{Chapter 4}

\section{Linear List r-hued Colorings of Graphs with Bounded Maximum Subgraph Average Degrees}

\subsection{Main Results}

We determine the linear list $r$-hued chromatic number for trees, complete $k$-partite graphs. In the last section, we also determine the linear list 2-hued chromatic number for graphs with maximum degree at most 4 and graphs whose maximum subgraph average degree and girth are in a certain ranges.

\subsection{The Linear $r$-hued Chromatic Number of Certain Graph Families}

Throughout this section, $r>0$ denotes an integer. In this section, we determine the linear $r$-hued chromatic number of a certain families of graphs, including complete bipartite graphs, and cycles. Proposition 4.2.1 below follows immediately from the definitions.

Proposition 4.2.1. Let $s \geq 1$ be an integer and $G$ be a nontrivial connected graph with $\Delta=$ $\Delta(G)$. Each of the following holds:

(i) $\chi_{\Delta+s}^{\ell}(G)=\chi_{\Delta}^{\ell}(G) \geq \cdots \geq \chi_{r}^{\ell}(G) \geq \chi_{r-1}^{\ell}(G) \geq \cdots \geq \chi_{2}^{\ell}(G) \geq \chi^{\ell}(G)$ and $\chi_{L, \Delta+s}^{\ell}(G)=$ $\chi_{L, \Delta}^{\ell}(G) \geq \cdots \geq \chi_{L, r}^{\ell}(G) \geq \chi_{L, r-1}^{\ell}(G) \geq \cdots \geq \chi_{L, 2}^{\ell}(G) \geq \chi_{L}^{\ell}(G)$.

(ii) $|V(G)| \geq \chi_{L, r}^{\ell}(G) \geq \chi_{r}^{\ell}(G) \geq \max \left\{\chi_{r}(G), \chi^{\ell}(G)\right\} \geq \chi(G)$.

Lemma 4.2.2. (Lai et al. , Theorem 2.2 of [57]) If $G$ is a tree with $|V(G)| \geq 3$, then $\chi_{r}(G)=$ $\min \{r, \Delta(G)\}+1$. 
Lemma 4.2.3. (Esperet, Montassier and Raspaud [33]) If $G$ is a tree with maximum degree $\Delta(G)$, then $\chi^{\ell}(G)=\left\lceil\frac{\Delta(G)}{2}\right\rceil+1$.

Lemma 4.2.4. Let $n \geq 2$ be an integer. If $G=K_{1, n-1}$, then $\chi_{r}^{\ell}(G)=\max \left\{\chi_{r}(G), \chi^{\ell}(G)\right\}$.

Proof. By Proposition 4.2.1(ii), $\chi_{r}^{\ell}(G) \geq \max \left\{\chi_{r}(G), \chi^{\ell}(G)\right\}$. We will show that $\chi_{r}^{\ell}(G) \leq$ $\max \left\{\chi_{r}(G), \chi^{\ell}(G)\right\}$ then. By Proposition 4.2.1(i), we may assume that $r \leq n-1$. By Lemmas 4.2.2 and 4.2.3, we observe that $\chi^{\ell}(G)=\left\lceil\frac{n-1}{2}\right\rceil+1$ and $\chi_{r}(G)=\min \{r, n-1\}+1$. Let $k_{1}=\left\lceil\frac{n-1}{2}\right\rceil+1$ and $k_{2}=r+1$.

If $r \leq\left\lceil\frac{n-1}{2}\right\rceil$, then we have $\max \left\{\chi_{r}(G), \chi^{\ell}(G)\right\}=k_{1}$ by Lemmas 4.2.2 and 4.2.3, and so $G$ has a linear $k_{1}$-coloring $c_{1}$. As $r \leq\left\lceil\frac{n-1}{2}\right\rceil, c_{1}$ is a linear $\left(k_{1}, r\right)$-coloring of $G$. If $\left\lceil\frac{n-1}{2}\right\rceil+1 \leq r \leq n-1$, then $\max \left\{\chi_{r}(G), \chi^{\ell}(G)\right\}=k_{2}$, and so $G$ has a linear $k_{2}$-coloring $c_{2}$. As $\left\lceil\frac{n-1}{2}\right\rceil+1 \leq r \leq n-1, c_{2}$ is a linear $\left(k_{2}, r\right)$-coloring of $G$. Thus in any case, $\chi_{r}^{\ell}(G) \leq \max \left\{\chi_{r}(G), \chi^{\ell}(G)\right\}$, which justifies the lemma.

Theorem 4.2.5. If $G$ is a tree with $|V(G)| \geq 3$, then $\chi_{r}^{\ell}(G)=\max \left\{\chi_{r}(G), \chi^{\ell}(G)\right\}$.

Proof. We argue by induction on $n=|V(G)|$. The theorem holds trivially if $n \leq 2$. If $n=3$, then $G=K_{1,2}$ and so the theorem follows from Lemma 4.2.4. Hence we assume that $G$ is a tree on $n \geq 4$ vertices and that the theorem holds for smaller values of $n$. By Lemma 4.2.4, we assume that $G \neq K_{1, n-1}$.

Let $v$ be a vertex of degree 1 in $G$ such that the degree of its neighbor is minimized, and let $u$ be the only vertex adjacent to $v$ in $G$. By induction, $\chi_{r}^{\ell}(G-v)=k=\max \left\{\chi_{r}(G-v), \chi^{\ell}(G-v)\right\}$.

Since $G \neq K_{1, n-1}$, and we choose $v$ such that $\left|N_{G}(u)\right|$ is minimized, we have $\Delta(G-$ $v)=\Delta(G)$. By induction, $G-v$ has a linear $(k, r)$-coloring $c^{\prime}: V(G-v) \mapsto \bar{k}$, where $k=$ $\max \left\{\chi_{r}(G-v), \chi^{\ell}(G-v)\right\}$. By Lemmas 4.2.2 and 4.2.3, and since $\Delta(G-v)=\Delta(G)$, we have $k=\max \left\{\chi_{r}(G), \chi^{\ell}(G)\right\}$.

Since $\left|N_{G}(u)\right| \leq \Delta(G)$, by Lemmas 4.2 .2 and 4.2 .3 , there must be a color $i_{0} \in \bar{k}$ such that at most one vertex in $N_{G-v}(u)$ is colored with $i_{0}$ under $c^{\prime}$ and such that $c^{\prime}(u) \neq i_{0}$. Further more, if $k \geq\left|c^{\prime}\left(N_{G-v}(u)\right)\right|+2$, then we choose such an $i_{0} \in \bar{k}-\left(c^{\prime}\left(N_{G-v}(u)\right) \cup\left\{c^{\prime}(u)\right\}\right)$. Define $c: V(G) \mapsto \bar{k}$ by $c(z)=c^{\prime}(z)$ if $z \neq v$ and $c(v)=i_{0}$.

Case 1. $r \leq\left|N_{G-v}(u)\right|$.

Then $c$ also satisfies the $r$-hued condition (C2). Since $i_{0}$ occurs in the neighbors of $u$ in $G$ at most twice, and since $c^{\prime}$ satisfies (1.1), $c$ also satisfies (1.1), and so in this case, $c$ is a linear $(k, r)$-coloring of $G$.

Case 2. $r>\left|N_{G-v}(u)\right|$.

We claim that we always have $k \geq\left|c^{\prime}\left(N_{G-v}(u)\right)\right|+2$. In fact, by induction and by Lemmas 4.2.2 and 4.2.3, if $r \geq \Delta(G)$, then $k=\max \left\{\chi_{r}(G-v), \chi^{\ell}(G-v)\right\}=\Delta(G)+1 \geq\left|c^{\prime}\left(N_{G-v}(u)\right)\right|+$ 
2 ; if $\Delta(G)>r$, then $k=\max \left\{r,\left\lceil\frac{n-1}{2}\right\rceil\right\}+1$, and so by $r>\left|N_{G-v}(u)\right|$, we also have $k \geq$ $\left|c^{\prime}\left(N_{G-v}(u)\right)\right|+2$. This justifies the claim.

By this claim, there is always a color $i_{0} \in \bar{k}-\left(c^{\prime}\left(N_{G-v}(u)\right) \cup\left\{c^{\prime}(u)\right\}\right)$, and so $c$ is a linear $(k, r)$-coloring of $G$ in this case also. This completes the proof of the theorem.

Next we determine the linear list $r$-hued chromatic number of complete bipartite graphs. To this aim, we need the help of the following two former results.

Theorem 4.2.6. (Esperet, Montassier and Raspaud, Proposition 3 of [33]) If $m \geq n \geq 1$ are integers, then $\chi_{L}^{\ell}\left(K_{m, n}\right)=\chi^{\ell}\left(K_{m, n}\right)=\lceil m / 2\rceil+n$.

Theorem 4.2.7. (Lai et al. , Theorem 3 of [57]) Suppose that $m \geq n \geq 2$, then $\chi_{r}\left(K_{m, n}\right)=$ $\min \{2 r, n+m, r+n\}$.

Theorem 4.2.8. Suppose that $m \geq n \geq 2$, then

$$
\chi_{L, r}^{\ell}\left(K_{m, n}\right)= \begin{cases}n+m, & m \leq r \\ r+n, & r<m<2 r \\ n+\left\lceil\frac{m}{2}\right\rceil, & m \geq 2 r .\end{cases}
$$

Proof. Let $(X, Y)$ denote the vertex bipartition of $K_{m, n}$ with $X=\left\{x_{1}, x_{2}, \ldots, x_{m}\right\}$ and $Y=\left\{y_{1}, y_{2}, \ldots, y_{n}\right\}$. In the rest of the proof, we assume that $k^{\prime} \geq k \geq 1$ are integers, and for given values of $k$, let $L: V\left(K_{m, n}\right) \mapsto 2^{\overline{k^{\prime}}}$ be an arbitrary list assignment to the vertices with $|L(v)| \geq k$, for every $v \in V\left(K_{m, n}\right)$.

If $m \leq r$, then let $k=\chi_{L, r}^{\ell}\left(K_{m, n}\right)$, and $c: V\left(K_{m, n}\right) \mapsto \bar{k}$ be a proper linear $(k, r)$-coloring. For any $i=1,2, \ldots, m$, by $(\mathrm{C} 2),\left|c\left(N_{G}\left(x_{i}\right)\right)\right|=n$ and so $|c(Y)|=n$. Similarly, $|c(X)|=m$. By (C1), for any $i$ with $1 \leq i \leq m$, and for any $j$ with $1 \leq j \leq n$, we have $c\left(x_{i}\right) \neq c\left(y_{j}\right)$, and so $k \geq m+n$. But since $\left|V\left(K_{m, n}\right)\right|=m+n$, it follows from Proposition 4.2.1(ii) that $k \leq m+n$. Thus if $m \leq r$, then $\chi_{L, r}^{\ell}\left(K_{m, n}\right)=m+n$. Hence we assume that $m>r$ and shall utilize the following algorithm.

Define

$$
k= \begin{cases}n+\left\lceil\frac{m}{2}\right\rceil & \text { if } m \geq 2 r . \\ n+r & \text { if } r<m<2 r .\end{cases}
$$

We present a linear $(L, r)$-coloring of $K_{m, n}$ first. Define a coloring $c: V\left(K_{m, n}\right) \mapsto \bigcup_{v \in V\left(K_{m, n}\right)} L(v)$ as follows. Firstly, for each $y_{i} \in Y$, choose $c\left(y_{i}\right) \in L\left(y_{i}\right)$ so that $|c(Y)|=n$. Since $k>n$, this can be done.

To color vertices in $X$, we randomly pick $c\left(x_{1}\right) \in L\left(x_{1}\right)-c(Y)$. If $m \geq 2 r$, then let $m^{\prime}=\left\lceil\frac{m}{2}\right\rceil$, and choose $c\left(x_{m^{\prime}+1}\right) \in L\left(x_{m^{\prime}+1}\right)-c(Y)$; for $2 \leq j \leq \cdots\left\lceil\frac{m}{2}\right\rceil$, pick $c\left(x_{j}\right) \in L\left(x_{j}\right)-$ $\left(c(Y) \cup\left\{c\left(x_{1}\right), \cdots, c\left(x_{j-1}\right)\right\}\right)$ and $c\left(x_{m^{\prime}+j}\right) \in L\left(x_{m^{\prime}+j}\right)-\left(c(Y) \cup\left\{c\left(x_{m^{\prime}+1}\right), \cdots, c\left(x_{m^{\prime}+j-1}\right)\right\}\right)$. 
If $r<m<2 r$, then choose $c\left(x_{r+1}\right) \in L\left(x_{r+1}\right)-c(Y)$; for $2 \leq j \leq \cdots r$, pick $c\left(x_{j}\right) \in L\left(x_{j}\right)-$ $\left(c(Y) \cup\left\{c\left(x_{1}\right), \cdots, c\left(x_{j-1}\right)\right\}\right)$ and $c\left(x_{r+j}\right) \in L\left(x_{r+j}\right)-\left(c(Y) \cup\left\{c\left(x_{r+1}\right), \ldots, c\left(x_{r+j-1}\right)\right\}\right)$. By (4.2), this coloring process can be done. Thus for every vertex $x_{i} \in X$, the colors of neighbors of $x_{i}$ are mutually distinct; and for every vertex $y_{j} \in Y$, any color can occur in the neighbors of $y_{j}$ at most twice. It follows by definition that $c$ is a linear $(L, r)$-coloring of $K_{m, n}$, and so by definition, $\chi_{L, r}^{\ell}\left(K_{m, n}\right) \leq k$.

To prove (6.2), we note that if $m \geq 2 r$, then by Proposition 4.2.1(i) and Theorem 4.2.6, $\chi_{L, r}^{\ell}\left(K_{m, n}\right) \geq \chi_{r}^{\ell}\left(K_{m, n}\right) \geq \chi^{\ell}\left(K_{m, n}\right)=n+\left\lceil\frac{m}{2}\right\rceil$; if $n<r$, then by Proposition 4.2.1(ii) and Theorem 4.2.7, $\chi_{L, r}^{\ell}\left(K_{m, n}\right) \geq \chi_{r}^{\ell}\left(K_{m, n}\right) \geq \chi_{r}\left(K_{m, n}\right)=n+r$.

It remains to show that if $n \geq r$, then $\chi_{L, r}^{\ell}\left(K_{m, n}\right) \geq n+r$. Let $k_{0}=\chi_{L, r}^{\ell}\left(K_{m, n}\right)$, and $c: V\left(K_{m, n}\right) \mapsto \overline{k_{0}}$ be a linear $\left(k_{0}, r\right)$-coloring of $K_{m, n}$. By (1.1), either $|c(X)|=m$ or $|c(Y)|=n$, as otherwise, there would be a bicolored cycle of length four. Moreover, to meet the requirement in (C2), if $|c(X)|=m$, then $|c(Y)| \geq r$; and if $|c(Y)|=n$, then $|c(X)| \geq r$. It follows that $\chi_{L, r}^{\ell}\left(K_{m, n}\right) \geq \chi_{r}^{\ell}\left(K_{m, n}\right) \geq r+n$. This completes the proof of the theorem.

Theorem 4.2.9. Suppose that $n_{1} \geq n_{2} \geq \cdots \geq n_{m}>0$ are integers. If $m \geq r+1$, then $\chi_{L, r}^{\ell}\left(K_{n_{1}, \ldots, n_{m}}\right)=\sum_{i=1}^{m} n_{i}-\left\lfloor\frac{n_{1}}{2}\right\rfloor$.

Proof. Let $\left(V_{1}, V_{2}, \ldots, V_{m}\right)$ denote the partition of $K_{n_{1}, \ldots, n_{m}}$ with $\left|V_{i}\right|=n_{i}$ for $1 \leq i \leq m$. Let $k_{1}=\chi_{L, r}^{\ell}\left(K_{n_{1}, \ldots, n_{m}}\right)$. For integers $k^{\prime} \geq k_{1} \geq 1$, let $L: V\left(K_{n_{1}, \ldots, n_{m}}\right) \mapsto 2^{\bar{k}^{\prime}}$ be an arbitrary $k_{1}$-list, and let $c$ be a linear $(L, r)$-coloring of $K_{n_{1}, \ldots, n_{m}}$.

Since $c$ is a linear coloring of $K_{n_{1}, \ldots, n_{m}}$, if for some $j$ with $1 \leq j \leq m$, there exist two vertices $v, v^{\prime} \in V_{j}$ such that $c(v)=c\left(v^{\prime}\right)$, then for any $i \neq j$, we must have $\left|c\left(V_{i}\right)\right|=\left|V_{i}\right|=n_{i}$. This is because that if we also have $u, u^{\prime} \in V_{i}$ with $c(u)=c\left(u^{\prime}\right)$, then $\left\{u, u^{\prime}, v, v^{\prime}\right\}$ will induce a bicolored cycle of length four, violating the assumption that $c$ is a linear coloring. As every color can occur at most twice in the vertices of $V_{j}$, it follows that

$$
\chi_{L, r}^{\ell}\left(K_{n_{1}, \ldots, n_{m}}\right)=k_{1} \geq \sum_{i=1}^{m} n_{i}-\left\lfloor\frac{n_{j}}{2}\right\rfloor \geq \sum_{i=1}^{m} n_{i}-\left\lfloor\frac{n_{1}}{2}\right\rfloor .
$$

Now, let $k_{2}=\sum_{i=1}^{m} n_{i}-\left\lfloor\frac{n_{1}}{2}\right\rfloor$. For integers $k^{\prime} \geq k_{2} \geq 1$, let $L: V\left(K_{n_{1}, \ldots, n_{m}}\right) \mapsto 2^{\bar{k}^{\prime}}$ be an arbitrary list assignment to the vertices with $|L(v)| \geq k_{2}$. We shall present a linear $(L, r)$-coloring of $K_{n_{1}, \ldots, n_{m}}$ as follows. First color the vertices in $\bigcup_{i=2}^{m} V_{i}$ such that $\left|c\left(\bigcup_{i=2}^{m} V_{i}\right)\right|=\left|\bigcup_{i=2}^{m} V_{i}\right|=$ $\sum_{i=2}^{m} n_{i}$. Since $k_{2}=\sum_{i=1}^{m} n_{i}-\left\lfloor\frac{n_{1}}{2}\right\rfloor$, such a coloring $c$ on $\bigcup_{i=2}^{m} V_{i}$ can be found. Next, we color the vertices in $V_{1}$. Denote $V_{1}=\left\{v_{1}, v_{2}, \cdots, v_{n_{1}}\right\}$, and let $t=\left\lceil\frac{n_{1}}{2}\right\rceil$. Randomly set $c\left(v_{1}\right) \in$ $L\left(v_{1}\right)-c\left(\bigcup_{i=2}^{m} V_{i}\right)$ and $c\left(v_{t+1}\right) \in L\left(v_{t+1}\right)-c\left(\bigcup_{i=2}^{m} V_{i}\right)$. For $2 \leq j \leq t$, choose $c\left(v_{j}\right) \in L\left(v_{j}\right)-$ $\left(c\left(\bigcup_{i=2}^{m} V_{i}\right) \cup\left\{c\left(v_{1}\right) \cdots, c\left(v_{j-1}\right)\right\}\right)$ and $c\left(v_{t+j}\right) \in L\left(v_{t+j}\right)-\left(c\left(\bigcup_{i=2}^{m} V_{i}\right) \cup\left\{c\left(v_{t+1}\right) \cdots, c\left(v_{t+j-1}\right)\right\}\right)$. As each color can occur in the vertices of $V_{1}$ at most twice, this is a linear coloring. Since 
$m \geq r+1$, the neighbors of each vertex in $V\left(K_{n_{1}, \ldots, n_{m}}\right)$ will be colored with at least $r$ different colors. Hence $(\mathrm{C} 2)$ is satisfied, and so $c$ is a linear $(L, r)$-coloring. It follows by definition that $\chi_{L, r}^{\ell}\left(K_{n_{1}, \ldots, n_{m}}\right) \leq k_{2}$. This, together with (4.3), implies the theorem.

Next, we determine the linear list $r$-hued chromatic number of cycles. The following lemma will be used.

Lemma 4.2.10. Suppose that $n \geq 3$ and $r \geq 2$ are integers. Then

$$
\chi_{L, r}\left(C_{n}\right)= \begin{cases}3, & n \equiv 0(\bmod 3) ; \\ 5, & n=5 ; \\ 4, & \text { otherwise. }\end{cases}
$$

In [2], Lemma 4.2.10 was proved for the case when $r=2$. Since $C_{n}$ is a 2-regular graph, the proof for the general case when $r \geq 2$ is similar and will be omitted.

Proposition 4.2.11. If $n \geq 3$ is a natural number, then the following holds:

$$
\chi_{L, r}^{\ell}\left(C_{n}\right)= \begin{cases}3, & n \equiv 0(\bmod 3) \\ 5, & n=5 \\ 4, & \text { otherwise. }\end{cases}
$$

Proof. By Proposition 4.2.1(i), $\chi_{L, 2}^{\ell}\left(C_{n}\right) \geq \chi_{L, 2}\left(C_{n}\right)$, and let $L$ be a list assigning color sets to each vertex of $C_{n}$. Since any proper subgraph of $C_{n}$ is a path, it follows that when $r \geq 2$, any $(L, r)$-coloring of $C_{n}$ must also be a linear $(L, r)$-coloring. Thus $\chi_{L, r}^{\ell}\left(C_{n}\right)=\chi_{L, r}\left(C_{n}\right)$, and so the proposition follows from Lemma 4.2.10.

\subsection{Linear 2-hued Colorings of Graphs with Bounded Average Degree}

In this section, we shall determine the linear 2-hued chromatic number of graphs with maximum degree at most 4 , or with maximum subgraph average degree not too large.

Let $G$ be a graph with $V=V(G)$, and let $V^{\prime} \subseteq V$ be a vertex subset. As in [11], $G\left[V^{\prime}\right]$ is the subgraph of $G$ induced by $V^{\prime}$. A mapping $c: V^{\prime} \mapsto \bigcup_{v \in V(G)} L(v)$ is a partial linear $(L, r)$-coloring of $G$ if $c$ is a linear $(L, r)$-coloring of $G\left[V^{\prime}\right]$. The set $C=\bigcup_{v \in V(G)} L(v)$ is referred to as the color set. The subgraph $G\left[V^{\prime}\right]$ is the support of the partial linear $(L, r)$-coloring $c$. Suppose that $c$ is a partial linear coloring of a graph $G$ with support $G^{\prime}$ using the color set $C$. For convenience, we also refer $V^{\prime}$ as the support of $c$. If a vertex $u$ in $G$ is not in the support of $c$, then we define $c(v)=\{\emptyset\}$. For each vertex $v \in V(G)$, we use $c_{G}^{2}(v)$ to denote the subset of colors each of which appears exactly twice on $N_{G}(v)$ under $c$. This notation will be used frequently throughout this section. We start with some lemmas and former results. 
Lemma 4.3.1. Let $k, r>0$ be integers, $G$ be a graph with minimum degree $\delta=\delta(G)$, and let $L$ be a $k$-list of $G$. If $\delta \geq 2 r-1$, then each of the following holds.

(i) Every linear $k$-coloring of $G$ is also a linear $(k, r)$-hued coloring of $G$. Consequently, $\chi_{r}^{\ell}(G)=$ $\chi^{\ell}(G)$.

(ii) Every linear L-coloring of $G$ is also a linear $(L, r)$-hued coloring of $G$. Consequently, $\chi_{L, r}^{\ell}(G)=\chi_{L}^{\ell}(G)$.

Proof. By Proposition 4.2.1, $\chi_{r}^{\ell}(G) \geq \chi^{\ell}(G)$ and $\chi_{L, r}^{\ell}(G) \geq \chi_{L}^{\ell}(G)$. Suppose that $G$ has a linear $k$-coloring $c$. Assume further that when $L$ is given, $c$ is a linear $L$-coloring of $G$. By the definition of linear coloring, $\left|c\left(N_{G}(v)\right)\right| \geq\left\lceil\frac{d(v)}{2}\right\rceil \geq\left\lceil\frac{\delta}{2}\right\rceil \geq\left\lceil\frac{2 r-1}{2}\right\rceil \geq r=\min \{d(v), r\}$. It follows that $c$ is also an $r$-hued coloring of $G$, and so $\chi_{r}^{\ell}(G)=\chi^{\ell}(G)$, and $\chi_{L, r}^{\ell}(G)=\chi_{L}^{\ell}(G)$.

Theorem 4.3.2. (Liu and $Y u$, Theorem 2 of [65]) If $G$ is a graph with $\Delta \leq 3$ which has no component isomorphic to $K_{3,3}$ or $C_{5}$, then $\chi_{L}^{\ell}(G) \leq 4$.

Lemma 4.3.3. (Esperet, Montassier and Raspaud [33]) If $G$ is a graph with maximum degree $\Delta \leq 4$, then $\chi^{\ell}(G) \leq 8$.

Theorem 4.3.4. If $G$ is a graph with maximum degree $\Delta \leq 4$, then $\chi_{2}^{\ell}(G) \leq 8$.

Proof. We prove the theorem by induction on $|V(G)|$. If $|V(G)| \leq 8$, the result holds trivially. Assume that $G$ is a graph with $\Delta(G) \leq 4$ and $|V(G)| \geq 9$. If $3 \leq \delta(G) \leq 4$, then by Lemmas 4.3.1 and 4.3.3, $\chi_{2}^{\ell}(G)=\chi^{\ell}(G) \leq 8$. Hence we assume that $\delta \leq 2$.

If $D_{1}(G) \neq \emptyset$, then pick $v \in D_{1}(G)$, let $u$ be the only neighbor of $v$ in $G$ and $G^{\prime}=G-v$. Thus $\Delta\left(G^{\prime}\right) \leq \Delta(G) \leq 4$, and $\left|V\left(G^{\prime}\right)\right|<|V(G)|$. By induction, $G^{\prime}$ has a linear (8,2)-coloring $c: V(G) \mapsto \overline{8}$. Since $\Delta(G) \leq 4,\left|c(u) \cup c_{G^{\prime}}^{2}(u)\right| \leq 1+\Delta(G) / 2=3$, and so we pick a color $c(v) \in \overline{8}-\left(c(u) \cup c_{G^{\prime}}^{2}(u)\right)$ and extend $c$ from $V\left(G^{\prime}\right)$ to $V(G)$. By the choice of $c(v)$ in this case and by definition, $c$ is a linear $(8,2)$-coloring.

Hence we may assume that $\delta=2$. Let $v \in D_{2}(G)$, and let $x$ and $y$ be the neighbors of $v$. Define $H$ to be the graph obtained from $G-v$ by adding a new edge $x y$ if it does not already exist. By the definition of $H, \Delta(H)=\Delta(G) \leq 4$ and $|V(H)|<|V(G)|$. By induction, there exists a linear $(8,2)$-coloring $c: V(H) \mapsto \overline{8}$ with $c(x) \neq c(y)$. Since $\Delta \leq 4$, we have $\left|\{c(x), c(y)\} \cup c_{H}^{2}(x) \cup c_{H}^{2}(y)\right| \leq 2+2 \cdot \frac{\Delta}{2}=6$, and so we can extend $c$ by setting $c(v) \in \overline{8} \backslash\{c(x), c(y)\} \cup C_{2}(x) \cup C_{2}(y)$. By definition, the extended $c$ is a linear $(8,2)$-coloring of $G$. This completes the proof of the theorem.

Let $G$ be a graph. The maximum subgraph average degree of $G$, denoted by $M a d(G)$, is defined by

$$
\operatorname{Mad}(G)=\max \left\{\frac{2|E(H)|}{|V(H)|}, H \subseteq G\right\} .
$$

To prove the next result, we need some more lemmas. 
Lemma 4.3.5. Let $k \geq 3$ and $r>0$ be integers, and $G$ be a graph with maximum degree $\Delta$. Suppose that $v \in D_{1}(G)$ and $k \geq \max \{\lceil\Delta / 2\rceil+1, r+1\}$. Each of the following holds.

(i) If $\chi_{r}^{\ell}(G-v)=k$, then $\chi_{r}^{\ell}(G) \leq k$.

(ii) If $\chi_{L, r}^{\ell}(G-v)=k$, then $\chi_{L, r}^{\ell}(G) \leq k$.

Proof. We shall prove (ii). The argument for the proof of (i) is similar and will be omitted. Let $u$ be the only neighbor of $v$ in $G, G^{\prime}=G-v, N_{G^{\prime}}(u)$ denote the set of vertices adjacent to $u$ in $G^{\prime}$. Suppose that $L$ is a $k$-list of $G$ and let $c$ be linear $(L, r)$-coloring of $G^{\prime}$. If $d_{G}(u) \geq r+1$, then $\mid c\left(N_{G^{\prime}}(u) \mid \geq r\right.$. And $\left|c_{G^{\prime}}^{2}(u)\right| \leq \frac{d_{G}(u)-1}{2} \leq \frac{\Delta}{2}-\frac{1}{2} \leq\lceil\Delta / 2\rceil-\frac{1}{2} \leq k-2$. Extend $c$ to a $k$-coloring of $G$ by defining $c(v) \in L(v)-\left(c_{G^{\prime}}^{2}(u) \cup\{c(u)\}\right)$. This is possible as $|L(v)| \geq k>\left|c_{G^{\prime}}^{2}(u) \cup\{c(u)\}\right|$. By the choice of $c(v)$ and since $c$ is a linear coloring of $G^{\prime}$, we conclude that $c$ is a linear $(L, r)$ coloring of $G$. Otherwise, $d_{G}(u) \leq r$. Then since $c$ is an $(L, r)$-coloring of $G^{\prime}$, by (C2), we have $\left|c\left(N_{G^{\prime}}(u)\right)\right|=d_{G}(u)-1 \leq r-1$ and $c_{G^{\prime}}^{2}(u)=\emptyset$. Extend $c$ to a $k$-coloring of $G$ by defining $c(v) \in L(v)-\left(c\left(N_{G^{\prime}}(u)\right) \cup\{c(u)\}\right)$. This is possible as $|L(v)| \geq k>\left|c\left(N_{G^{\prime}}(u)\right) \cup\{c(u)\}\right|$. By the choice of $c(v)$, we have $\left|c\left(N_{G}(u)\right)\right|=d_{G}(u)$, and so (C2) is also satisfied. This justifies the lemma.

For any path $P=v_{0} v_{1} v_{2} \cdots v_{p}, P^{o}=V(P)-\left\{v_{0}, v_{p}\right\}$ denote the set of all internal vertices of $P$. A path $P$ of $G$ is a divalent path of $G$ if $V(P) \subseteq G\left[D_{2}(G)\right]$ and $v_{0} \neq v_{p}$; and is internally divalent if $P^{o} \subseteq D_{2}(G)$. We shall take the following convention in our arguments below: If $L$ is a $k$-list of $G$, then for a subgraph $G^{\prime}$ of $G$, we also use $L$ to denote the restriction of $L$ to $V\left(G^{\prime}\right)$.

Lemma 4.3.6. Let $G$ be a graph with maximum degree $\Delta$. Suppose that $v \in D_{1}(G)$ and $k, q, r>0$ be integers such that $k \geq \max \{\lceil\Delta / 2\rceil+q, r+q, 5\}$. Let $L$ be a k-list of $G$. Let $P=u_{1} u_{2} \cdots u_{p}$ be a divalent path of $G$ satisfying $p+q=5$, and let $G^{\prime}=G-V(P)$. If $1 \leq q \leq 3$, then each of the following holds.

(i) If $\left.\chi_{r}^{\ell}\left(G^{\prime}\right)\right)=k$, then $\chi_{r}^{\ell}(G) \leq k$.

(ii) If $\chi_{L, r}^{\ell}\left(G^{\prime}\right)=k$, then $\chi_{L, r}^{\ell}(G) \leq k$.

Proof. We only prove Part (ii), as the proof for Part (i) is similar, and will be omitted.

Let $Q=u_{0} u_{1} u_{2} \cdots u_{p} u_{p+1}$ be a path in $G$ such that $P=Q-\left\{u_{0}, u_{p+1}\right\}$ is a divalent path of $G$, and let $G^{\prime}=G-V(P)$. Assume that $c$ is a linear $(L, r)$-coloring of $G^{\prime}$. We then will extend $c$ to a linear $(L, r)$-coloring of $G$.

Recall that $c_{G^{\prime}}^{2}(v)$ is the set of colors that occur twice in $c\left(N_{G^{\prime}}(v)\right)$. Since $c$ is a linear coloring of $G^{\prime}$, for $v \in\left\{u_{0}, u_{p+1}\right\}$, we have $\left|c_{G^{\prime}}^{2}(v)\right| \leq \frac{d_{G^{\prime}}(v)}{2} \leq \frac{d_{G}(v)-1}{2} \leq\lceil\Delta / 2\rceil-\frac{1}{2}$. As $\lceil\Delta / 2\rceil$ is an integer, we have $\left|c_{G^{\prime}}^{2}(v)\right| \leq\lceil\Delta / 2\rceil-1$.

In the following, for each case of $1 \leq q \leq 3$, we will define an extension of $c$ to a coloring (also denoted by $c$, for notational convenience) of $G$. After this is done, we shall show that the extended $c$ is indeed a linear $(L, r)$-coloring of $G$. 
By assumption,

$$
\left|L\left(u_{1}\right)\right| \geq k \geq(\lceil\Delta / 2\rceil-1)+5-p \geq\left|c_{G^{\prime}}^{2}(v)\right|+5-p .
$$

Assume that $q=1$, and so $p=4$ and $\left|L\left(u_{1}\right)\right| \geq\left|c_{G^{\prime}}^{2}\left(u_{0}\right) \cup\left\{c\left(u_{0}\right)\right\}\right|$. Define,

$$
c\left(u_{1}\right) \in \begin{cases}L\left(u_{1}\right)-\left(c_{G^{\prime}}^{2}\left(u_{0}\right) \cup\left\{c\left(u_{0}\right)\right\}\right) & \text { if } d_{G}\left(u_{0}\right) \geq r+1, \\ L\left(u_{1}\right)-\left(c\left(N_{G^{\prime}}\left(u_{0}\right)\right) \cup\left\{c\left(u_{0}\right)\right\}\right) & \text { if } d_{G}\left(u_{0}\right) \leq r .\end{cases}
$$

As $d_{G}\left(u_{0}\right) \geq r+1$ implies $\left|c\left(N_{G^{\prime}}\left(u_{0}\right)\right)\right| \geq r$, and as $d_{G}\left(u_{0}\right) \leq r$ implies $\left|c\left(N_{G^{\prime}}\left(u_{0}\right)\right)\right|=d_{G}\left(u_{0}\right)-$ $1 \leq r-1$ by $(\mathrm{C} 2)$. All vertices in $N_{G}\left(u_{0}\right) \cup\left\{u_{0}\right\}$ satisfies the $r$-hued condition (C2). Similarly, we define

$$
c\left(u_{4}\right) \in \begin{cases}L\left(u_{4}\right)-\left(c_{G^{\prime}}^{2}\left(u_{5}\right) \cup\left\{c\left(u_{5}\right)\right\}\right) & \text { if } d_{G}\left(u_{5}\right) \geq r+1, \\ L\left(u_{4}\right)-\left(c\left(N_{G^{\prime}}\left(u_{5}\right)\right) \cup\left\{c\left(u_{5}\right)\right\}\right) & \text { if } d_{G}\left(u_{5}\right) \leq r .\end{cases}
$$

After $c\left(u_{1}\right), c\left(u_{4}\right)$ are defined, by $k \geq 5$, we choose $c\left(u_{2}\right) \in L\left(u_{2}\right)-\left\{c\left(u_{0}\right), c\left(u_{1}\right), c\left(u_{4}\right)\right\}$, and then $c\left(u_{3}\right) \in L\left(u_{3}\right)-\left\{c\left(u_{1}\right), c\left(u_{2}\right), c\left(u_{4}\right), c\left(u_{5}\right)\right\}$.

Assume that $q=2$, and so $p=3$ and $\left|L\left(u_{1}\right)\right| \geq\left|c_{G^{\prime}}^{2}\left(u_{0}\right) \cup\left\{c\left(u_{0}\right)\right\}\right|$. Define

$$
c\left(u_{1}\right) \in \begin{cases}L\left(u_{1}\right)-\left(c_{G^{\prime}}^{2}\left(u_{0}\right) \cup\left\{c\left(u_{0}\right)\right\}\right) & \text { if } d_{G}\left(u_{0}\right) \geq r+1, \\ L\left(u_{1}\right)-\left(c\left(N_{G^{\prime}}\left(u_{0}\right) \cup\left\{c\left(u_{0}\right)\right\}\right)\right. & \text { if } d_{G}\left(u_{0}\right) \leq r .\end{cases}
$$

Similarly, we define

$$
c\left(u_{3}\right) \in \begin{cases}L\left(u_{3}\right)-\left(c_{G^{\prime}}^{2}\left(u_{4}\right) \cup\left\{c\left(u_{1}\right), c\left(u_{4}\right)\right\}\right) & \text { if } d_{G}\left(u_{0}\right) \geq r+1, \\ L\left(u_{3}\right)-\left(c\left(N_{G^{\prime}}\left(u_{4}\right)\right) \cup\left\{c\left(u_{1}\right), c\left(u_{4}\right)\right\}\right) & \text { if } d_{G}\left(u_{0}\right) \leq r .\end{cases}
$$

After $c\left(u_{1}\right), c\left(u_{3}\right)$ are defined, by $k \geq 5$, we choose $c\left(u_{2}\right) \in L\left(u_{2}\right)-\left\{c\left(u_{0}\right), c\left(u_{1}\right), c\left(u_{3}\right), c\left(u_{4}\right)\right\}$.

Assume that $q=3$, and so $p=2$ and $\left|L\left(u_{1}\right)\right| \geq\left|c_{G^{\prime}}^{2}\left(u_{0}\right) \cup\left\{c\left(u_{0}\right), c\left(u_{3}\right)\right\}\right|$. Define

$$
c\left(u_{1}\right) \in \begin{cases}L\left(u_{1}\right)-\left(c_{G^{\prime}}^{2}\left(u_{0}\right) \cup\left\{c\left(u_{0}\right), c\left(u_{3}\right)\right\}\right) & \text { if } d_{G}\left(u_{0}\right) \geq r+1, \\ L\left(u_{1}\right)-\left(c\left(N_{G^{\prime}}\left(u_{0}\right)\right) \cup\left\{c\left(u_{0}\right), c\left(u_{3}\right)\right\}\right) & \text { if } d_{G}\left(u_{0}\right) \leq r .\end{cases}
$$

and

$$
c\left(u_{2}\right) \in \begin{cases}L\left(u_{2}\right)-\left(c_{G^{\prime}}^{2}\left(u_{3}\right) \cup\left\{c\left(u_{0}\right), c\left(u_{1}\right), c\left(u_{3}\right)\right\}\right) & \text { if } d_{G}\left(u_{2}\right) \geq r+1, \\ L\left(u_{2}\right)-\left(c\left(N_{G^{\prime}}\left(u_{3}\right)\right) \cup\left\{c\left(u_{0}\right), c\left(u_{1}\right), c\left(u_{3}\right)\right\}\right) & \text { if } d_{G}\left(u_{2}\right) \leq r .\end{cases}
$$

In any case, as $k \geq \max \{\lceil\Delta / 2\rceil+q, r+q, 5\}$, the extended colorings of $G$ are possible. In addition, we have $|c(V(P))|=|V(P)| \geq 2$ and as $c\left(u_{0}\right), c\left(u_{p+1}\right) \notin c(V(P))$. As $c$ is a linear coloring of $G^{\prime}$, by definition, the extended $c$ is a linear coloring of $G$. Similarly, as $c$ is an $(L, r)$-coloring of $G^{\prime}$, the extended $c$ is an $(L, r)$-coloring of $G$. This proves the lemma. 


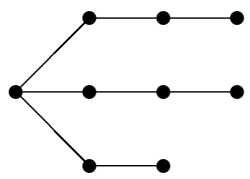

$Y_{3,3,2}$

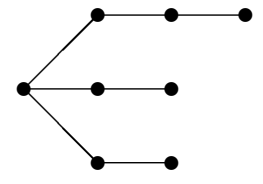

$Y_{3,2,2}$

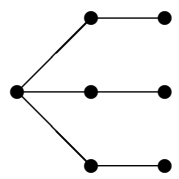

$Y_{2,2,2}$

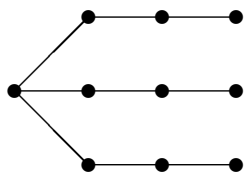

$Y_{3,3,3}$

Figure B: Examples of $Y_{s_{1}, s_{2}, s_{3}}$

For integers $s_{1} \geq s_{2} \geq s_{3} \geq 1$, let $Y_{s_{1}, s_{2}, s_{3}}$ be the graph obtained from disjoint paths $P_{s_{1}+1}, P_{s_{2}+1}$ and $P_{s_{3}+1}$ by identifying an end vertex of each of these three paths. (See examples depicted in Figure B). The only vertex of degree 3 in a $Y_{s_{1}, s_{2}, s_{3}}$ is called the center of it.

Let $q, r>0$ be integers with $q=1,2,3$. Define

$$
k^{*}= \begin{cases}\max \{\lceil\Delta / 2\rceil+q, r+q, 6\}, & \text { if } q=1,2 ; \\ \max \{\lceil\Delta / 2\rceil+3, r+3,7\}, & \text { otherwise. }\end{cases}
$$

Lemma 4.3.7. Let $k, q, r>0$ be integers with $k \geq k^{*}$ and $G$ be a graph with maximum degree $\Delta$. Let $L$ be a k-list of $G$. For $q=1,2,3$, Let $Y_{q}$ denote a subgraph of $G$ with center $w_{0}$ and $D_{1}\left(Y_{q}\right)=\left\{w_{1}, w_{2}, w_{3}\right\}$ such that $Y_{1}$ is isomorphic to $Y_{3,3,2}, Y_{2}$ is isomorphic to $Y_{3,2,2}, Y_{3}$ is isomorphic to $Y_{2,2,2}$ and $Y_{4}$ is isomorphic to $Y_{3,3,3}$, and such that, for $1 \leq j \leq 4$, every $\left(w_{0}, w_{j}\right)$ path of $Y_{q}$ is an internally divalent path of $G$. Let $G^{\prime}=G-V\left(Y_{q}-D_{1}\left(Y_{q}\right)\right)$. Then for $q=1,2,3$, each of the following holds.

(i) If $\chi_{r}^{\ell}\left(G^{\prime}\right)=k$, then $\chi_{r}^{\ell}(G) \leq k$.

(ii) If $\chi_{L, r}^{\ell}\left(G^{\prime}\right)=k$, then $\chi_{L, r}^{\ell}(G) \leq k$.

Proof. We shall prove Part (ii) only as the proof for Part (i) is similar and will be omitted. For each value $q=1,2,3$, let $c$ be a linear $(L, r)$-coloring of $G^{\prime}$. We shall extend $c$ to a linear $(L, r)$ coloring, also denoted by $c$, of $G$, to prove the lemma. For $1 \leq i \leq 3$, let $P_{i}=w_{0} u_{1}^{i} \cdots u_{s_{i}-1}^{i} w_{i}$ be a path in $Y_{s_{1}, s_{2}, s_{3}}$ such that $u_{j}^{i} \in D_{2}(G)$ and $V\left(P_{i}\right) \cap V\left(P_{j}\right)=\left\{w_{0}\right\}, 1 \leq i<j \leq 3$. By assumption, $k>(\lceil\Delta / 2\rceil-1)+1$.

Assume that $q=1$. Then as $\left|c_{G^{\prime}}^{2}\left(w_{1}\right)\right| \leq\lceil\Delta / 2\rceil-1,\left|L\left(u_{2}^{1}\right)\right| \geq k>\left|c_{G^{\prime}}^{2}\left(w_{1}\right) \cup\left\{c\left(w_{1}\right)\right\}\right|$. Define

$$
c\left(u_{2}^{1}\right) \in \begin{cases}L\left(u_{2}^{1}\right)-\left(c_{G^{\prime}}^{2}\left(w_{1}\right) \cup\left\{c\left(w_{1}\right)\right\}\right) & \text { if } d_{G}\left(w_{1}\right) \geq r+1, \\ L\left(u_{2}^{1}\right)-\left(c\left(N_{G^{\prime}}\left(w_{1}\right)\right) \cup\left\{c\left(w_{1}\right)\right\}\right) & \text { if } d_{G}\left(w_{1}\right) \leq r .\end{cases}
$$

Similarly, as $k \geq \max \{\lceil\Delta / 2\rceil+1, r+1,6\}$, define

$$
\begin{aligned}
& c\left(u_{2}^{2}\right) \in \begin{cases}L\left(u_{2}^{2}\right)-\left(c_{G^{\prime}}^{2}\left(w_{2}\right) \cup\left\{c\left(w_{2}\right)\right\}\right) & \text { if } d_{G}\left(w_{2}\right) \geq r+1, \\
L\left(u_{2}^{2}\right)-\left(c\left(N_{G^{\prime}}\left(w_{2}\right)\right) \cup\left\{c\left(w_{2}\right)\right\}\right) & \text { if } d_{G}\left(w_{2}\right) \leq r,\end{cases} \\
& c\left(u_{1}^{3}\right) \in \begin{cases}L\left(u_{1}^{3}\right)-\left(c_{G^{\prime}}^{2}\left(w_{3}\right) \cup\left\{c\left(w_{3}\right)\right\}\right) & \text { if } d_{G}\left(w_{3}\right) \geq r+1, \\
L\left(u_{1}^{3}\right)-\left(c\left(N_{G^{\prime}}\left(w_{3}\right)\right) \cup\left\{c\left(w_{3}\right)\right\}\right) & \text { if } d_{G}\left(w_{3}\right) \leq r,\end{cases}
\end{aligned}
$$


and $c\left(w_{0}\right) \in L\left(w_{0}\right)-\left\{c\left(u_{2}^{1}\right), c\left(u_{2}^{2}\right), c\left(u_{1}^{3}\right), c\left(w_{3}\right)\right\}$. After $c\left(u_{2}^{1}\right), c\left(u_{2}^{2}\right), c\left(u_{1}^{3}\right)$ and $c\left(w_{0}\right)$ are defined, we choose $c\left(u_{1}^{1}\right) \in L\left(u_{1}^{1}\right)-\left\{c\left(u_{2}^{1}\right), c\left(u_{1}^{3}\right), c\left(w_{1}\right), c\left(w_{0}\right)\right\}$, and $c\left(u_{1}^{2}\right) \in L\left(u_{1}^{2}\right)-\left\{c\left(u_{2}^{2}\right), c\left(u_{1}^{1}\right)\right.$, $\left.c\left(w_{2}\right), c\left(u_{1}^{3}\right), c\left(w_{0}\right)\right\}$.

Assume that $q=2$. Since $\left|L\left(u_{2}^{1}\right)\right| \geq k>(\lceil\Delta / 2\rceil-1)+1 \geq\left|c_{G^{\prime}}^{2}\left(w_{1}\right) \cup\left\{c\left(w_{1}\right)\right\}\right|$, we can define $c\left(u_{2}^{1}\right)$ the same as in (4.6). Similarly, define

$$
\begin{gathered}
c\left(u_{1}^{2}\right) \in \begin{cases}L\left(u_{1}^{2}\right)-\left(c_{G^{\prime}}^{2}\left(w_{2}\right) \cup\left\{c\left(w_{2}\right)\right\}\right) & \text { if } d_{G}\left(w_{2}\right) \geq r+1, \\
L\left(u_{1}^{2}\right)-\left(c\left(N_{G^{\prime}}\left(w_{2}\right)\right) \cup\left\{c\left(w_{2}\right)\right\}\right) & \text { if } d_{G}\left(w_{2}\right) \leq r,\end{cases} \\
c\left(u_{1}^{3}\right) \in \begin{cases}L\left(u_{1}^{3}\right)-\left(c_{G^{\prime}}^{2}\left(w_{3}\right) \cup\left\{c\left(w_{3}\right), c\left(u_{1}^{2}\right)\right\}\right) & \text { if } d_{G}\left(w_{3}\right) \geq r+1, \\
L\left(u_{1}^{3}\right)-\left(c\left(N_{G^{\prime}}\left(w_{3}\right)\right) \cup\left\{c\left(w_{3}\right), c\left(u_{1}^{2}\right)\right\}\right) & \text { if } d_{G}\left(w_{3}\right) \leq r,\end{cases}
\end{gathered}
$$

After $c\left(u_{2}^{1}\right), c\left(u_{1}^{2}\right)$ and $c\left(u_{1}^{3}\right)$ are defined, as $k \geq \max \{\lceil\Delta / 2\rceil+2, r+2,6\}$, we can find $c\left(w_{0}\right) \in$ $L\left(w_{0}\right)-\left\{c\left(u_{2}^{1}\right), c\left(u_{1}^{2}\right), c\left(u_{1}^{3}\right), c\left(w_{2}\right), c\left(w_{3}\right)\right\}$ and $c\left(u_{1}^{1}\right) \in L\left(u_{1}^{1}\right)-\left\{c\left(w_{0}\right), c\left(w_{1}\right), c\left(u_{2}^{1}\right), c\left(u_{1}^{2}\right), c\left(u_{1}^{3}\right)\right\}$ to complete the extension of $c$.

Assume that $q=3$. We define

$$
\begin{gathered}
c\left(u_{1}^{1}\right) \in \begin{cases}L\left(u_{1}^{1}\right)-\left(c_{G^{\prime}}^{2}\left(w_{1}\right) \cup\left\{c\left(w_{1}\right)\right\}\right) & \text { if } d_{G}\left(w_{1}\right) \geq r+1, \\
L\left(u_{1}^{1}\right)-\left(c\left(N_{G^{\prime}}\left(w_{1}\right)\right) \cup\left\{c\left(w_{1}\right)\right\}\right) & \text { if } d_{G}\left(w_{1}\right) \leq r,\end{cases} \\
c\left(u_{1}^{2}\right) \in \begin{cases}L\left(u_{1}^{2}\right)-\left(c_{G^{\prime}}^{2}\left(w_{2}\right) \cup\left\{c\left(w_{2}\right), c\left(u_{1}^{1}\right)\right\}\right) & \text { if } d_{G}\left(w_{2}\right) \geq r+1, \\
L\left(u_{1}^{2}\right)-\left(c\left(N_{G^{\prime}}\left(w_{2}\right)\right) \cup\left\{c\left(w_{2}\right), c\left(u_{1}^{1}\right)\right\}\right) & \text { if } d_{G}\left(w_{2}\right) \leq r,\end{cases} \\
c\left(u_{1}^{3}\right) \in \begin{cases}L\left(u_{1}^{3}\right)-\left(c_{G^{\prime}}^{2}\left(w_{3}\right) \cup\left\{c\left(w_{3}\right), c\left(u_{1}^{1}\right), c\left(u_{1}^{2}\right)\right\}\right) & \text { if } d_{G}\left(w_{3}\right) \geq r+1, \\
L\left(u_{1}^{3}\right)-\left(c\left(N_{G^{\prime}}\left(w_{3}\right)\right) \cup\left\{c\left(w_{3}\right), c\left(u_{1}^{1}\right), c\left(u_{1}^{2}\right)\right\}\right) & \text { if } d_{G}\left(w_{3}\right) \leq r,\end{cases}
\end{gathered}
$$

As $k \geq 7$, we choose $c\left(w_{0}\right) \in L\left(w_{0}\right)-\left\{c\left(u_{1}^{1}\right), c\left(u_{1}^{2}\right), c\left(u_{1}^{3}\right), c\left(w_{1}\right), c\left(w_{2}\right), c\left(w_{3}\right)\right\}$.

Assume that $q=4$. For $j=1,2,3$, as $\left|c_{G^{\prime}}^{2}\left(w_{j}\right)\right| \leq\lceil\Delta / 2\rceil-1,\left|L\left(u_{2}^{j}\right)\right| \geq k>\mid c_{G^{\prime}}^{2}\left(w_{j}\right) \cup$ $\left\{c\left(w_{j}\right)\right\} \mid$, define

$$
c\left(u_{2}^{j}\right) \in \begin{cases}L\left(u_{2}^{j}\right)-\left(c_{G^{\prime}}^{2}\left(w_{j}\right) \cup\left\{c\left(w_{j}\right)\right\}\right) & \text { if } d_{G}\left(w_{j}\right) \geq r+1, \\ L\left(u_{2}^{j}\right)-\left(c\left(N_{G^{\prime}}\left(w_{j}\right)\right) \cup\left\{c\left(w_{j}\right)\right\}\right) & \text { if } d_{G}\left(w_{j}\right) \leq r .\end{cases}
$$

Next, we pick $c\left(w_{0}\right) \in L\left(w_{0}\right)-\left\{c\left(u_{2}^{1}\right), c\left(u_{2}^{2}\right), c\left(u_{2}^{3}\right)\right\}$. After $c\left(u_{2}^{1}\right), c\left(u_{2}^{2}\right), c\left(u_{2}^{3}\right)$ and $c\left(w_{0}\right)$ are defined, we choose $c\left(u_{1}^{1}\right) \in L\left(u_{1}^{1}\right)-\left\{c\left(u_{2}^{1}\right), c\left(w_{1}\right), c\left(w_{0}\right)\right\}, c\left(u_{1}^{2}\right) \in L\left(u_{1}^{2}\right)-\left\{c\left(u_{2}^{2}\right), c\left(u_{1}^{1}\right), c\left(w_{2}\right)\right.$, $\left.c\left(w_{0}\right)\right\}$. and $c\left(u_{1}^{3}\right) \in L\left(u_{1}^{3}\right)-\left\{c\left(u_{2}^{3}\right), c\left(u_{1}^{1}\right), c\left(u_{1}^{2}\right), c\left(w_{3}\right), c\left(w_{0}\right)\right\}$.

Now in any case when $q=1,2,3,4$, we have obtained an extended $(L, r)$-coloring $c$ of $G$. Since $c$ is a linear coloring of $G^{\prime}$ and since, for each $1 \leq i \leq 4,\left|\left\{c\left(w_{0}\right), c\left(u_{1}^{i}\right), c\left(u_{2}^{i}\right)\right\}\right|=3$, it follows by definition that the extended $c$ is a linear coloring of $G$. Since $c$ satisfies $(\mathrm{C} 2)$ in $G^{\prime}$, by the definition of the extended $c$, the extended $c$ also satisfied (C2). Hence $c$ is a linear $(L, r)$-coloring of $G$. 
Theorem 4.3.8. Let $G$ be a graph with maximum degree $\Delta$ :

(i) If $\Delta \geq 9$ and $\operatorname{Mad}(G)<\frac{7}{3}$, then $\chi_{L, r}^{\ell}(G) \leq \max \{\lceil\Delta / 2\rceil+1, r+1\}$.

(ii) If $\Delta \geq 7$ and $\operatorname{Mad}(G)<\frac{12}{5}$, then $\chi_{L, r}^{\ell}(G) \leq \max \{\lceil\Delta / 2\rceil+2, r+2\}$.

(iii) If $\Delta \geq 7$ and $\operatorname{Mad}(G)<\frac{5}{2}$, then $\chi_{L, r}^{\ell}(G) \leq \max \{\lceil\Delta / 2\rceil+3, r+3\}$.

Let $g(G)$ be the girth of a graph $G$. Since every planar or projective-planar graph $G$ verifies $\operatorname{Mad}(G)<2 g(G) /(g(G)-2)$, we obtain the following corollary:

Corollary 4.3.9. Let $G$ be a planar or projective-planar graph with maximum degree $\Delta$ :

(i) If $\Delta \geq 9$ and $g(G) \geq 14$, then $\chi_{L, r}^{\ell}(G) \leq \max \{\lceil\Delta / 2\rceil+1, r+1\}$.

(ii) If $\Delta \geq 7$ and $g(G) \geq 12$, then $\chi_{L, r}^{\ell}(G) \leq \max \{\lceil\Delta / 2\rceil+2, r+2\}$.

(iii) If $\Delta \geq 7$ and $g(G) \geq 10$, then $\chi_{L, r}^{\ell}(G) \leq \max \{\lceil\Delta / 2\rceil+3, r+3\}$.

Proof of Theorem 4.3.8. We argue by contradiction and assume that

$G$ be a counterexample to the theorem with $|V(G)|$ minimized.

By (4.7) and Lemma 4.3.5, we may assume that $\delta(G) \geq 2$.

(i) Since $G$ is a counterexample, there exists a $k$-list with $k \geq \max \{\lceil\Delta / 2\rceil+1, r+1\} \geq 6$ such that $G$ does not have a linear $(L, r)$-coloring.

Claim 1. Each of the following holds.

(C1.1) $G$ does not have a divalent path of length 3 .

(C1.2) $G$ does not have a divalent path of length 2 with one of the endpoints being adjacent to a vertex of degree at most 4 .

(C1.3) $G$ does not have an induced subgraph $H_{1}$ consisting of three internally divalent paths $P_{1}, P_{2}$ and $P_{3}$, such that $\left|E\left(P_{1}\right)\right|=\left|E\left(P_{2}\right)\right|=3,\left|E\left(P_{3}\right)\right| \in\{2,3\}$ and such that for some $w \in D_{3}(G)$, and for any $1 \leq i<j \leq 3, V\left(P_{i}\right) \cap V\left(P_{j}\right)=\{w\}$.

Proof. (C1.1) If $G$ contains a divalent path $P_{4}=v_{1} v_{2} v_{3} v_{4}$, then let $G^{\prime}=G-V\left(P_{4}\right)$. As $G^{\prime}$ is a subgraph of $G$, we have $\operatorname{Mad}\left(G^{\prime}\right)<\frac{16}{7}$. By $(4.7), G^{\prime}$ has a linear $(L, r)$-coloring. By Lemma 4.3.6, $G$ has a linear $(L, r)$-coloring of $G$, contrary to $(4.7)$.

(C1.2) Suppose that $G$ contains a path $P=v_{0} v_{1} v_{2} v_{3} v_{4}$ such that $P_{3}=v_{1} v_{2} v_{3}$ is a divalent path with $d\left(v_{0}\right) \leq 4$. Let $G^{\prime}=G-\left\{v_{1}, v_{2}\right\}$. As $G^{\prime}$ is a subgraph of $G$, we have $\operatorname{Mad}\left(G^{\prime}\right)<\frac{16}{7}$. By (4.7), $G^{\prime}$ has a linear $(L, r)$-coloring. Since $k \geq 6$ and $d\left(v_{0}\right) \leq 4$, we can find $c\left(v_{1}\right) \in$ $L\left(v_{1}\right)-\left\{c\left(N\left(v_{0}\right) \backslash\left\{v_{1}\right\}\right), c\left(v_{0}\right), c\left(v_{3}\right)\right\}, c\left(v_{2}\right) \in L\left(v_{2}\right)-\left\{c\left(v_{0}\right), c\left(v_{1}\right), c\left(v_{3}\right), c\left(v_{4}\right)\right\}$. By definition, the extended $c$ is a linear $(L, r)$-coloring of $G$, contrary to (4.7).

(C1.3) Suppose that $G$ has such an induced $H_{1}$, which is isomorphic to a $Y_{3,3,2}$ or a $Y_{3,3,3}$. It follows from (4.7) and Lemma 4.3.7 that $G$ would have a linear $(L, r)$-coloring of $G$, contrary to (4.7). This proves Claim 1. 
For $v \in V(G), n_{2}(v)=\left|D_{2}(G) \cap N_{G}(v)\right|$. For a vertex $u \in D_{2}(G)$ and for $i=0,1,2, u$ is called a Type- $(i+1)$ vertex if $n_{2}(u)=i$. We complete the proof by applying a discharging method to find a contradiction. Set the initial charge $w_{0}(v)=d(v)$ for each vertex $v \in V(G)$. We then apply the following discharging rules:

$\mathbf{R} 1$ Each $3^{+}$-vertex gives $\frac{1}{6}$ to each adjacent Type- 1 vertex and $\frac{1}{3}$ to each adjacent Type- 2 vertex. R2 Each $5^{+}$-vertex gives $\frac{1}{6}$ to each adjacent Type-3 vertex via the adjacent 2 -vertex.

Let $w(v)$ denote the new charge at a vertex $v$ after carrying out these discharging rules. We shall show that $w(v) \geq \frac{7}{3}$ for every $v \in V(G)$ by analyzing the following cases.

(1) $d(v)=2$. Let $N(v)=\{x, y\}$ with $d(x) \leq d(y)$. If $v$ is a Type- 1 vertex, then each of $x$ and $y$ gives $\frac{1}{6}$ to $v$ by $\mathrm{R} 1$, and so $w(v) \geq d(v)+2 \cdot \frac{1}{6}=\frac{7}{3}$. If $v$ is a Type- 2 vertex, then $d(y) \geq 3$ and $y$ gives $\frac{1}{3}$ to $v$ by $\mathrm{R} 1$. Hence, $w(v) \geq d(v)+\frac{1}{3}=\frac{7}{3}$. If $v$ is a Type- 3 vertex, then $d(x)=d(y)=2$ and each of $x$ and $y$ is adjacent to a $5^{+}$-vertex by $(\mathrm{C} 1.2)$. It follows that $w(v) \geq d(v)+2 \cdot \frac{1}{6}=\frac{7}{3}$ by R2.

(2) $d(v)=3$. By $(\mathrm{C} 1.3), v$ is adjacent to at most two Type- 2 vertices. If $v$ is adjacent to at most one Type-2 vertex, then $w(v) \geq d(v)-\frac{1}{3}-2 \cdot \frac{1}{6}=\frac{7}{3}$ by R1. Otherwise, $v$ is not adjacent to any Type- 1 vertex by (C1.3). Hence, $w(v) \geq d(v)-2 \cdot \frac{1}{3}=\frac{7}{3}$ by $\mathrm{R} 1$.

(3) $d(v) \geq 4$. If $d(v)=4$, then $v$ gives at most four times of $\frac{1}{3}$ by R 1 and so $w(v) \geq d(v)-4 \cdot \frac{1}{3}=\frac{8}{3}$. If $d(v) \geq 5$, then $w(v) \geq d(v)-d(v) \cdot\left(\frac{1}{3}+\frac{1}{6}\right)=\frac{d(v)}{2} \geq \frac{5}{2}$ by $\mathrm{R} 1$ and $\mathrm{R} 2$.

Therefore, $w(v) \geq \frac{7}{3}$ for every vertex. Since

$$
\sum_{v \in V(G)} w(v)=\sum_{v \in V(G)} w_{0}(v)=\sum_{v \in V(G)} d(v)=2|E(G)|,
$$

we have $\operatorname{Mad}(G) \geq \frac{2|E(G)|}{|V(G)|}=\frac{\sum_{v \in V(G)} w(v)}{|V(G)|} \geq \frac{7}{3}$, contrary to the assumption that $\operatorname{Mad}(G)<$ $\frac{7}{3}$.

(ii) Since $G$ is a counterexample, there exists a $k$-list with $k \geq \max \{\lceil\Delta / 2\rceil+2, r+2\} \geq 6$ such that $G$ does not have a linear $(L, r)$-coloring. Using arguments similar to those in the proof of Claim 1, we have the following claim.

Claim 2. Each of the following holds.

(C2.1) $G$ does not have a divalent path of length 2 .

(C2.2) $G$ does not have an induced subgraph $H_{2}$ consisting of three internally divalent paths $P_{1}, P_{2}$ and $P_{3}$, such that $\left|E\left(P_{1}\right)\right|=3,\left|E\left(P_{2}\right)\right|=\left|E\left(P_{3}\right)\right|=2$ and such that for some $w \in D_{3}(G)$, and for any $1 \leq i<j \leq 3, V\left(P_{i}\right) \cap V\left(P_{j}\right)=\{w\}$.

Once again we set the initial charge $w_{0}(v)=d(v)$ for each vertex $v$ and apply the following discharging rule.

R3 Each $3^{+}$-vertex gives $\frac{1}{5}$ to each adjacent Type- 1 vertex and $\frac{2}{5}$ to Type- 2 vertex. 
Let $w(v)$ denote the new charge after recharging by R3. We will show that $w(v) \geq \frac{12}{5}$ for all $v \in V(G)$.

(1) If $d(v)=2$, then $n_{2}(v) \leq 1$ by $(\mathrm{C} 2.1)$. If $n_{2}(v)=1$, then $v$ is a Type- 2 vertex and receives $\frac{2}{5}$ from the adjacent $3^{+}$-vertex by R3. If $n_{2}(v)=0$, then $v$ is of Type- 1 and receives two times $\frac{1}{5}$ from the adjacent $3^{+}$-vertices by R3. Thus, $w(v) \geq d(v)+\frac{2}{5}=\frac{12}{5}$.

(2) If $d(v)=3$, then $v$ is adjacent to at most one Type-2 vertex by (C2.2). If $v$ is adjacent to one Type- 2 vertex, then $v$ is adjacent to at most one Type- 1 vertex. It follows by R3 that $w(v) \geq d(v)-\frac{2}{5}-\frac{1}{5}=\frac{12}{5}$. Otherwise, $v$ is adjacent to at most three Type- 1 vertices, and so by $\mathrm{R} 3 w(v) \geq d(v)-3 \times \frac{1}{5}=\frac{12}{5}$.

(3) If $d(v) \geq 4$, then $v$ is adjacent to at most $d(v) 2$-vertices and so $v$ discharges at most $d(v) \cdot \frac{2}{5}$ to adjacent 2 -vertices. Thus, $w(v) \geq d(v)-d(v) \cdot \frac{2}{5}=\frac{3}{5} d(v) \geq \frac{12}{5}$.

In any case, $w(v) \geq \frac{12}{5}$ for every $v \in V(G)$. Since

$$
\sum_{v \in V(G)} w(v)=\sum_{v \in V(G)} w_{0}(v)=\sum_{v \in V(G)} d(v)=2|E(G)|,
$$

we have obtained a contradiction:

$$
\frac{12}{5}>\operatorname{Mad}(G) \geq \frac{2|E(G)|}{|V(G)|}=\frac{\sum_{v \in V(G)} w(v)}{|V(G)|} \geq \frac{12}{5} .
$$

(iii) Since $G$ is a counterexample, there exists a $k$-list with $k \geq \max \{\lceil\Delta / 2\rceil+3, r+3\} \geq 7$ such that $G$ does not have a linear $(L, r)$-coloring. Again by a similar argument in the proof of Claim 1 , we have the following claim.

Claim 3. Each of the following holds.

(C3.1) $G$ does not have a divalent path of length 1 .

(C3.2) $G$ does not have an induced subgraph $H_{3}$ consisting of three internally divalent paths $P_{1}, P_{2}$ and $P_{3}$, such that $\left|E\left(P_{1}\right)\right|=\left|E\left(P_{2}\right)\right|=\left|E\left(P_{3}\right)\right|=2$ and such that for some $w \in D_{3}(G)$, and for any $1 \leq i<j \leq 3, V\left(P_{i}\right) \cap V\left(P_{j}\right)=\{w\}$.

We start with our initial charge $w_{0}(v)=d(v)$ for each vertex $v \in V(G)$, and then apply the following discharging rule.

$\mathbf{R} 4$ Each $3^{+}$-vertex gives $\frac{1}{4}$ to each adjacent 2 -vertex.

Let $w(v)$ be the new charge after discharging rule $\mathrm{R} 4$. We will show that $w(v) \geq \frac{5}{2}$ for every $v \in V(G)$.

(1) If $d(v)=2$, then $v$ is adjacent to two $3^{+}$-vertices by (C3.1) and receives two times $\frac{1}{4}$ from the adjacent $3^{+}$-vertices by $\mathrm{R} 4$. It follows that $w(v) \geq 2+2 \cdot \frac{1}{4}=\frac{5}{2}$.

(2) If $d(v)=3$, then $v$ is adjacent to at most two 2-vertices by (C3.2). By R4, $w(v) \geq 3-2 \cdot \frac{1}{4}=\frac{5}{2}$.

(3) If $d(v) \geq 4$, then $v$ is adjacent to at most $d(v) 2$-vertices, so $w(v) \geq d(v)-d(v) \cdot \frac{1}{4}=\frac{3}{4} d(v) \geq 3$. 
Therefore, in any case, $w(v) \geq \frac{5}{2}$ for every vertex. Since

$$
\sum_{v \in V(G)} w(v)=\sum_{v \in V(G)} w_{0}(v)=\sum_{v \in V(G)} d(v)=2|E(G)|,
$$

we have obtained a contradiction:

$$
\frac{5}{2}>\operatorname{Mad}(G) \geq \frac{2|E(G)|}{|V(G)|}=\frac{\sum_{v \in V(G)} w(v)}{|V(G)|} \geq \frac{5}{2} .
$$

This completes the proof of the theorem. 


\section{Chapter 5}

\section{Linear List $r$-hued Coloring of $K_{4}$-minor Free Graphs}

\subsection{Main Results}

Define $f(\Delta, r)=\max \{r,\lceil\Delta / 2\rceil\}+\lceil\Delta / 2\rceil+2$. The main results of this chapter are the following.

Theorem 5.1.1. Let $G$ be a $K_{4}$-minor free graph with $\Delta=\Delta(G)$, and $r \geq 2$ be an integer. Then $\chi_{L, r}^{\ell}(G) \leq f(\Delta, r)$.

Theorem 5.1.2. If $G$ is a planar graph with $\Delta=\Delta(G)$, then $\chi_{L, 2}^{\ell}(G) \leq \Delta+7$.

These results will be proved in the subsequent sections.

\subsection{Proof of Theorem 5.1.1}

Let $G$ be a graph with the vertex set $V=V(G)$ with $V^{\prime} \subset V$ being a vertex subset. A mapping $c: V^{\prime} \longrightarrow \bigcup_{v \in V^{\prime}} L(v)$ is a partial $(L, r)$-coloring if $c$ is a linear $(L, r)$-coloring of $G\left[V^{\prime}\right]$. For each $v \in V(G)-V^{\prime}$, define $c(v)=\emptyset$, and for each vertex $v \in V(G)$, define $c_{G}^{2}(v)=\left\{i \in c\left(N_{G}(v)\right)\right.$ : for distinct $v_{1}, v_{2} \in N_{G}(v), c\left(v_{1}\right)=c\left(v_{2}\right)=i$ \}. For every vertex $v \in V^{\prime}$, define

$$
c[v]= \begin{cases}\{c(v)\} \cup c_{G}^{2}(v), & \text { if }\left|c\left(N_{G}(v)\right)\right| \geq r \\ \{c(v)\} \cup c(N(v)), & \text { otherwise. }\end{cases}
$$

Thus, given a partial $(L, r)$-coloring $c, c[v]$ consists of the set of colors that cannot be used for uncolored neighbors of $v$. For a vertex $v \in V(G)$ which has at least one uncolored neighbor by definition, $\left|c_{G}^{2}(v)\right| \leq\lfloor(\Delta-1) / 2\rfloor=\lceil\Delta / 2\rceil-1$, and so by $(5.1)$,

$$
|c[v]| \leq \max \{r,\lceil\Delta / 2\rceil\} .
$$


Define $S_{G}(u)=\left\{x\right.$ : either $d_{G}(x) \geq 3$ with $u x \in E(G)$ or for some $w \in D_{2}(G), u w, w x \in$ $E(G)\}$. Let $s_{G}(u)=\left|S_{G}(u)\right|$.

It is well known [26] that every $K_{4}$-minor free graph contains a vertex of degree at most two. Lih et al. [66] proved the following lemma.

Lemma 5.2.1. (Lih, Wang and Zhu [66]) Let $G$ be a $K_{4}$-minor free graph. Then one of the following conditions holds:

(i) $\delta(G) \leq 1$.

(ii) There exists two adjacent 2-vertices.

(iii) There exists a vertex $u$ with $d(u) \geq 3$ such that $s_{G}(u) \leq 2$.

Proof of Theorem 5.1.1. We argue by contradiction to prove Theorem 5.1.1. Assume that

$G$ is a counterexample to theorem 5.1.1 with $|V(G)|$ minimized.

Let $\Delta=\Delta(G)$, and $k=f(\Delta, r)$. By (5.3), there must be a $k$-list $L$ such that

$G$ does not have a linear $(L, r)$-coloring.

In the arguments below, we will obtain a $K_{4}$-minor free graph $H$ by making local modifications of $G$ such that $|V(H)|<|V(G)|$. By (5.3), $H$ has a linear $(L, r)$-coloring $c$. To obtain a contradiction, we shall extend and modify $c$ to a linear $(L, r)$-coloring of $G$.

Claim 1. $\delta(G)=2$.

By contradiction, assume that $x \in D_{1}(G)$ with $N_{G}(x)=\{u\}$. Define $H=G-x$. Then $H$ is also $K_{4}$-minor free and $|V(H)|<|V(G)|$. By (5.3), $H$ admits a linear $(L, r)$-coloring $c$. By (5.1), $|c[u]| \leq \max \{r,\lceil\Delta / 2\rceil\}<k \leq|L(x)|$. Therefore, $c$ can be extended to a linear $(L, r)$-coloring of $G$ by defining $c(x) \in L(x)-c[u]$, contrary to (5.4).

Claim 2. $D_{2}(G)$ is an independent set.

By contradiction, assume that for some $x, y \in D_{2}(G), x y \in E(G)$. Denote $N_{G}(x)=\{u, y\}$ and $N_{G}(y)=\{v, x\}$. Let $H=G-x+u y$ (if $u \neq v$ ) or $H=G-x$ if $\left(u=v\right.$ ). As $H$ is $K_{4}$-minor free with $|V(H)|<|V(G)|$, by (5.3), $H$ has a linear $(L, r)$-coloring $c$ with $S(c)=V(G)-\{x\}$. By (5.1), $c[y]=\{c(y), c(v)\}$. It follows that $|c[u] \cup c[y]| \leq|c[u]|+|c[y]| \leq \max \{r,\lceil\Delta / 2\rceil\}+2<k \leq$ $|L(x)|$. Thus, $c$ can be extend to a linear $(L, r)$-coloring of $G$ by defining $c(x) \in L(x)-(c[u] \cup c[y])$, contrary to $(5.4)$.

By Lemma 5.2.1 and Claims 1 and 2, $G$ contains a vertex $u$ with $d_{G}(u) \geq 3$ such that $1 \leq s_{G}(u) \leq 2$. In the rest of the proof, we always assume that $u$ is such a vertex. For each $x \in S_{G}(u)$, define

$$
M_{G}(u, x)=N_{G}(x) \cap N_{G}(u) \cap D_{2}(G) \text { and } m_{G}(x)=\left|M_{G}(u, x)\right| \text {. }
$$


Since $d_{G}(u) \geq 3$ such that $s_{G}(u) \leq 2$, there exists a $z \in S_{G}(u)$ with $m_{G}(z) \geq 1$. Throughout the rest of this section, we assume that $x$ is a vertex in $S_{G}(u)$ with $m_{G}(x) \geq 1$. We have the following claim.

Claim 3. $s_{G}(u)=2$.

If $m_{G}(x)=1$, then $m_{G}(y) \leq 1$. When $m_{G}(y)=0$, we have $u x, u y \in E(G)$; and either $u x \in E(G)$ or $u y \in E(G)$ when $m_{G}(y)=1$. Hence, without loss of generality, we may assume that $u x \in E(G)$. Thus, $|c[u] \cup c[x]|=|c[x]|+|c[u] \backslash c[x]| \leq d(x)+2<k$. Otherwise, $m_{G}(x) \geq 2$. If $u y \in E(G)$, then $m_{G}(x) \geq(d(u)-1) / 2$ and $|c[u] \cup c[x]|=|c[x]|+|c[u] \backslash c[x]| \leq d(x)+(d(u)-1-$ $\left.m_{G}(x)\right)+1 \leq d(x)+(d(u)-1) / 2+1 \leq 3 r / 2+1 / 2<k$. If $u y \neq E(G)$, then $m_{G}(x) \geq d(u) / 2$ and $|c[u] \cup c[x]|=|c[x]|+|c[u] \backslash c[x]| \leq d(x)+\left(d(u)-m_{G}(x)\right)+1 \leq d(x)+d(u) / 2+1 \leq 3 r / 2+1<k$.

Claim 4. Let $w \in M_{G}(u, x)$ and $c$ be a linear $(L, r)$-coloring of $G-w$ with $c(u) \neq c(x)$. Then $\max \left\{d_{G}(u), d_{G}(x)\right\} \leq r$.

By contradiction and assume that $\max \left\{d_{G}(u), d_{G}(x)\right\}=d_{G}(u)>r$. Since $w \in M_{G}(u, x)$, we have $d_{G-w}(u) \geq r$, then $c\left(N_{G-w}(u)\right) \geq r$. By (5.1), $|c[u]| \leq\lceil\Delta / 2\rceil$, and so $|c[u] \cup c[x]| \leq$ $|c[u]|+|c[x]| \leq\lceil\Delta / 2\rceil+\max \{r,\lceil\Delta / 2\rceil\}<k \leq|L(w)|$. As $c(u) \neq c(x)$ and $|c[u] \cup c[x]|<|L(w)|$, the partial linear $(L, r)$-coloring $c$ can be extended to a linear $(L, r)$-coloring of $G$ by choosing $c(w) \in L(w)-(c[u] \cup c[x])$, contrary to $(5.4)$.

Recall that $k=f(\Delta, r)$. We have the following claim.

Claim 5. $\lfloor 3 r / 2\rfloor+1<k$.

If $\lceil\Delta / 2\rceil \leq r \leq \Delta$, then $k=\max \{r,\lceil\Delta / 2\rceil\}+\lceil\Delta / 2\rceil+2=r+\lceil\Delta / 2\rceil+2$. Thus, $\lfloor 3 r / 2\rfloor+1=$ $r+\lfloor r / 2\rfloor+1 \leq r+\lceil\Delta / 2\rceil+1<k$. If $1 \leq r \leq\lceil\Delta / 2\rceil$, then $k=\max \{r,\lceil\Delta / 2\rceil\}+\lceil\Delta / 2\rceil+2=$ $2\lceil\Delta / 2\rceil+2$. It follows that $\lfloor 3 r / 2\rfloor+1 \leq 3 r / 2+1 \leq 3 / 2\lceil\Delta / 2\rceil+1<k$.

By Claim $3, s_{G}(u)=2$. Let $S_{G}(u)=\{x, y\}$. Then by the definition of $S_{G}(u)$, it follows that $N_{G}(u) \subseteq N_{G}(x) \cup N_{G}(y) \cup\{x, y\}$. Without loss of generality, we shall always assume that $m_{G}(x) \geq m_{G}(y)$. Since $s_{G}(u) \geq 3$, we have $m_{G}(x) \geq 1$. Pick $w \in M_{G}(u, x)$ and define $H=G-w$. By (5.3), $H$ has a linear $(L, r)$-coloring $c$. We now proceed the proof of Theorem 5.1 .1 by a case analysis.

Case 1. $x u \in E(G)$.

As $x u \in E(H), c(u) \neq c(x)$. By Claim 4, we have $\max \left\{d_{G}(u), d_{G}(x)\right\} \leq r$. Since $x \in N_{G}(u)$, we have $|c[u] \cup c[x]| \leq d_{G}(u)+d_{G}(x)-m_{G}(x)-1$. By $m_{G}(x)+m_{G}(y) \geq d_{G}(u)-2$ and by $m_{G}(x) \geq m_{G}(y)$, we conclude that $m_{G}(x) \geq\left\lceil\left(d_{G}(u)-2\right) / 2\right\rceil=\left\lceil d_{G}(u) / 2\right\rceil-1$. Hence

$$
\begin{aligned}
|c[u] \cup c[x]| & \leq d_{G}(u)+d_{G}(x)-m_{G}(x)-1 \leq d_{G}(u)+d_{G}(x)-\left\lceil d_{G}(u) / 2\right\rceil \\
& \leq\left\lfloor d_{G}(u) / 2\right\rfloor+d_{G}(x) \leq\lfloor 3 r / 2\rfloor<k \leq|L(w)| .
\end{aligned}
$$


As $c(u) \neq c(x), c$ can be extended to a linear $(L, r)$-coloring of $G$ by taking $c(w) \in L(w)-(c[u] \cup$ $c[x])$, contrary to (5.4). This proves Case 1 .

Case 2. Both $x u \notin E(G)$ and $y u \notin E(G)$.

Since $x u, y u \notin E(G)$ and $m_{G}(x) \geq m_{G}(y)$, we conclude that $m_{G}(x) \geq\left\lceil d_{G}(u) / 2\right\rceil \geq 2$. and so there exists a $w^{\prime} \in N_{H}(x) \cap N_{H}(u) \cap D_{2}(H)$, This implies that $c(u) \neq c(x)$. By Claim 4, we have $\max \left\{d_{G}(u), d_{G}(x)\right\} \leq r$. Since $x$ is not adjacent to $u$, we have $|c[u] \cup c[x]| \leq$ $d_{G}(u)+d_{G}(x)-m_{G}(x)+1$. Hence

$$
\begin{aligned}
|c[u] \cup c[x]| & \leq d_{G}(u)+d_{G}(x)-m_{G}(x)+1 \leq d_{G}(u)+d_{G}(x)-\left\lceil d_{G}(u) / 2\right\rceil+1 \\
& \leq\left\lfloor d_{G}(u) / 2\right\rfloor+d_{G}(x)+1 \leq\lfloor 3 r / 2\rfloor+1<k \leq|L(w)| .
\end{aligned}
$$

As $c(u) \neq c(x), c$ can be extended to a linear $(L, r)$-coloring of $G$ by choosing $c(w) \in L(w)-$ $(c[u] \cup c[x])$, contrary to $(5.4)$. This proves Case 2 .

Case 3. Both $x u \notin E(G)$ and $y u \in E(G)$.

If $m_{G}(x)=m_{G}(y)$, we may interchange $x$ and $y$, and so Case 3 becomes Case 1 . Hence we may assume that $m_{G}(x)>m_{G}(y)$.

Case 3.1. $d_{G}(u)$ is odd.

Since $d_{G}(u)$ is odd, $m_{G}(x)+m_{G}(y)=d_{G}(u)-1$ is even, and so $m_{G}(x) \geq m_{G}(y)+2 \geq 2$.

Case 3.1.1. $m_{G}(x) \geq m_{G}(y)+4$.

Since $m_{G}(x) \geq m_{G}(y)+4 \geq 4, M_{H}(u, x) \neq \emptyset$, and so $c(u) \neq c(x)$. By Claim 4 , we have $\max \left\{d_{G}(u), d_{G}(x)\right\} \leq r$. Hence,

$$
\begin{aligned}
|c[u] \cup c[x]| & \leq d_{G}(u)+d_{G}(x)-m_{G}(x)+1 \leq d_{G}(u)+d_{G}(x)-\left(d_{G}(u)+3\right) / 2+1 \\
& =\left\lfloor d_{G}(u) / 2\right\rfloor+d_{G}(x) \leq\lfloor 3 r / 2\rfloor<k \leq|L(w)| .
\end{aligned}
$$

As $c(u) \neq c(x), c$ can be extended to a linear $(L, r)$-coloring of $G$ by choosing $c(w) \in L(w)-$ $(c[u] \cup c[x])$, contrary to $(5.4)$.

Case 3.1.2. $m_{G}(x)=m_{G}(y)+2$.

If $m_{G}(x)=m_{G}(y)+2 \geq 2$, then $M_{H}(u, x) \neq \emptyset$, and so $c(u) \neq c(x)$. By Claim 4 , we have $\max \left\{d_{G}(u), d_{G}(x)\right\} \leq r$. If $d_{G}(u)<r$, then

$$
\begin{aligned}
|c[u] \cup c[x]| & \leq d_{G}(u)+d_{G}(x)-m_{G}(x)+1 \leq d_{G}(u)+d_{G}(x)-\left(d_{G}(u)+1\right) / 2+1 \\
& =\left(d_{G}(u)+1\right) / 2+d_{G}(x) \leq\lfloor r / 2\rfloor+d_{G}(x) \leq\lfloor 3 r / 2\rfloor<k \leq|L(w)| .
\end{aligned}
$$

Thus we assume that $d_{G}(u)=r$. If $x y \in E(G)$, then

$$
\begin{aligned}
|c[u] \cup c[x]| & \leq d_{G}(u)+d_{G}(x)-1-\left(m_{G}(x)-1\right) \leq d_{G}(u)+d_{G}(x)-\left(d_{G}(u)+1\right) / 2 \\
& =\left(d_{G}(u)-1\right) / 2+d_{G}(x) \leq\lfloor r / 2\rfloor+d_{G}(x) \\
& \leq\lfloor 3 r / 2\rfloor<k \leq|L(w)| .
\end{aligned}
$$


Next we assume that $d_{G}(u)=r$ and $x y \notin E(G)$. In this case,

$$
\begin{aligned}
|c[u] \cup c[x]| & \leq d_{G}(u)+d_{G}(x)-\left(m_{G}(x)-1\right) \leq d_{G}(u)+d_{G}(x)-\left(d_{G}(u)+1\right) / 2+1 \\
& =\left(d_{G}(u)-1\right) / 2+d_{G}(x)+1 \leq\lfloor r / 2\rfloor+d_{G}(x)+1 \\
& \leq\lfloor 3 r / 2\rfloor+1<k \leq|L(w)| .
\end{aligned}
$$

In any cases, as $c(u) \neq c(x), c$ can be extended to a linear $(L, r)$-coloring of $G$ by choosing $c(w) \in L(w)-(c[u] \cup c[x])$, contrary to (5.4). This proves Case 3.1.

Case 3.2. $d_{G}(u)$ is even.

Since $d_{G}(u)$ is even, $m_{G}(x)+m_{G}(y)=d_{G}(u)-1$ is odd and $m_{G}(x) \geq m_{G}(y)+1$.

If $m_{G}(x) \geq m_{G}(y)+3 \geq 3$, then $m_{H}(x) \geq 2$, and so $c(u) \neq c(x)$. By Claim 4 , we have $\max \left\{d_{G}(u), d_{G}(x)\right\} \leq r$, and so $|c[u] \cup c[x]| \leq d_{G}(u)+d_{G}(x)-m_{G}(x)+1 \leq d_{G}(x)+d_{G}(u) / 2 \leq$ $\lfloor 3 r / 2\rfloor+1<k \leq|L(w)|$. As $c(u) \neq c(x), c$ can be extended to a linear $(L, r)$-coloring of $G$ by choosing $c(w) \in L(w)-(c[u] \cup c[x])$, contrary to $(5.4)$.

Hence $m_{G}(x) \leq m_{G}(y)+2$. Since $m_{G}(x)+m_{G}(y)$ is odd, we may assume that $m_{G}(x)=$ $m_{G}(y)+1$. Since $d_{G}(u) \geq 4, m_{G}(y)=d_{G}(u) / 2-1 \geq 1$. Choose $w^{\prime} \in M_{G}(u, y)$ and let $H^{\prime \prime}=G-w^{\prime}$. By $(5.3), H^{\prime \prime}$ has an $(L, r)$-coloring $c$. As $u y \in E(G), c(u) \neq c(y)$. By Claim 4 , we have $\max \left\{d_{G}(u), d_{G}(y)\right\} \leq r$. Hence

$$
\begin{aligned}
|c[u] \cup c[y]| & \leq d_{G}(u)+d_{G}(y)-m_{G}(y)-1 \leq d_{G}(u)+d_{G}(y)-d_{G}(u) / 2 \\
& =d_{G}(u) / 2+d_{G}(y) \leq\lfloor 3 r / 2\rfloor<k \leq|L(w)| .
\end{aligned}
$$

It follows from $c(u) \neq c(x)$ that $c$ can be extended to a linear $(L, r)$-coloring of $G$ by defining $c(w) \in L(w)-(c[u] \cup c[x])$, contrary to (5.3). This completes the proof for Case 3.2.

Since every case leads to a contradiction, this establishes the theorem and completes the proof.

\subsection{Proof of Theorem 5.1.2}

The best know bound for linear chromatic number of planar graphs so far was obtained by Cai, Xie and Yang (see [14]) as shown in Theorem 5.3.1 below. In the proof of Theorem 5.3.1, the authors always count the number of available colors for uncolored vertex and never apply the technique of exchange colors. Hence the result can be extended to list version. The main purpose of this section is to extend Theorem 5.3.1 to linear $(L, 2)$-hued chromatic.

Theorem 5.3.1. (Cai, Xie and Yang, [14]) If $G$ is a planar graph with $\Delta=\Delta(G)$, then $\chi_{L}^{\ell}(G) \leq \Delta+7$.

The following lemma will be needed in our arguments. 
Lemma 5.3.2. (Fan et al. [35]) If a graph $G$ with minimum degree $\delta \geq 2 r-1$, then $\chi_{L, r}^{\ell}(G)=$ $\chi_{L}^{\ell}(G)$

Proof of Theorem 5.1.2. Let $G$ be a planar graph, and let $k=\Delta+7$. Suppose that $L$ is a $k$-list of $G$. We prove the theorem by induction on $|V(G)|$, and so we may assume that $G$ is connected. If $|V(G)| \leq \Delta+7$, then the theorem holds trivially. Hence assume that $G$ is a planar graph with $|V(G)| \geq \Delta+8$. As $G$ is a planar graph, $\delta(G) \leq 5$. If $3 \leq \delta(G) \leq 5$, then $\chi_{L, 2}^{\ell}(G)=\chi_{L}^{\ell}(G) \leq \Delta+7$ by Lemma 5.3.2. Next we assume that $1 \leq \delta(G) \leq 2$. In the arguments below, we will first obtain a partial $(L, 2)$-coloring $c$ of $G$. Then extend $c$ to an $(L, 2)$-coloring of $G$ to complete the inductive proof.

Case 1. $\delta(G)=1$.

Let $v \in D_{1}(G)$ with $N_{G}(v)=\{u\}$. Since $|V(G)| \geq \Delta+8$ and since $G$ is connected, we have $d_{G}(u) \geq 2$. By induction, $G-v$ has a linear $(L, 2)$-coloring $c$. By $(5.1),|c[u]| \leq \max \left\{2,\left\lceil\frac{\Delta}{2}\right\rceil\right\}<k$, and so $c$ can be extended to a linear $(L, 2)$-coloring of $G$ by assigning $c(v) \in L(v)-c[u]$.

Case 2. $\delta(G)=2$.

Let $v \in D_{2}(G)$ with $N_{G}(v)=\{x, y\}$. Let $H=(G-v)+x y$ (if $\left.x y \notin E(G)\right) H=G-v$ (if $x y \in$ $E(G))$. By induction, $H$ has a linear $(L, 2)$-coloring $c$. Thus $c$ is a partial $(k, 2)$-coloring of $G$. By (5.1), we have $|c[x] \cup c[y]| \leq|c[x]|+|c[y]| \leq \max \left\{2,\left\lceil\frac{\Delta}{2}\right\rceil\right\}+\max \left\{2,\left\lceil\frac{\Delta}{2}\right\rceil\right\} \leq \max \{4, \Delta+1\}<k$, and so $c$ can be extended to a linear $(L, 2)$-coloring of $G$ by defining $c(v) \in L(v)-(c[x] \cup c[y])$. This completes the proof of the theorem. 


\section{Chapter 6}

\section{Disjoint Spanning Arborescences in $k$-arc-strong Digraphs}

\subsection{Main Results}

In Section 6.2, we prove that $\lambda(D) \geq k$ if and only if for any vertex $v \in V(D), D$ has $k$-arc-disjoint spanning arborescences rooted at $v$, thereby obtaining a digraph result analogous to Theorem 1.3.1. Moreover, we determine the extremal value $\min \{|A(D)|: D \in \mathcal{A}(k)$ and $|V(D)|=n\}$. In Section 6.3 , we prove a characterization of uniformly dense digraphs analogous to the characterization of uniformly dense undirected graphs in [15].

\subsection{Relationship Between $k$-arc-strong Connectivity and Dis- joint Spanning Arborescences}

We start with some digraph families with certain properties arc-disjoint spanning arborescences. This section is mainly devoted to the study of the relationship between $\mathcal{D}(k)$ and the following seemingly different families of digraphs.

Definition 6.2.1. Let $T$ be an oriented tree with a fixed vertex $r \in V(T)$. $T$ is an outarborescence rooted at $r$ (or an $r^{+}$-arborescence) if $d_{T}^{-}(r)=0$ and for any $v \in V(T)-r$, $d_{T}^{-}(v)=1 ; T$ is an in-arborescence rooted at $r$ (or an $r^{-}$-arborescence) if $d_{T}^{+}(r)=0$ and for any $v \in V(T)-r, d_{T}^{+}(v)=1$. In either case, $r$ is called the root of $T$.

Thus an arborescence is an out-arborescence, and an $r$-arborescence is an $r^{+}$-arborescence. For any function $f: V(D) \rightarrow \mathbb{R}^{+}$and any subset $S \subseteq V(D)$, define $f(S)=\sum_{v \in S} f(v)$.

Definition 6.2.2. Let $k \geq s \geq 1$ be integers.

(i) Let $\mathcal{A}_{1}(k, s)$ be the family of digraphs such that $D \in \mathcal{A}_{1}(k, s)$ if and only if for any $S \subseteq V(D)$ 
with $|S| \leq s, D$ has $k$ arc-disjoint spanning out-arborescences whose roots are in $S$.

(ii) Let $\mathcal{A}_{2}(k, s)$ be the family of digraphs such that $D \in \mathcal{A}_{2}(k, s)$ if and only if for any $S \subseteq V(D)$ with $|S| \leq s, D$ has $k$ arc-disjoint spanning in-arborescences whose roots are in $S$.

(iii) Let $\mathcal{A}_{3}(k, s)$ be the family of digraphs such that $D \in \mathcal{A}_{3}(k, s)$ if and only if for any $u, l$ : $V(D) \rightarrow \mathbb{R}^{+}$with $u \geq l$, and for any $S \subseteq V(D)$ with $|S| \leq s$ and with $u(S) \geq k \geq l(S)$, D has $k$ arc-disjoint spanning out-arborescences whose roots are in $S$ in such a way that every $r \in S$ is the root of at least $l(r)$ and at most $u(r)$ of such spanning arborescences.

(iv) Let $\mathcal{A}_{4}(k, s)$ be the family of digraphs such that $D \in \mathcal{A}_{4}(k, s)$ if and only if for any $u, l$ : $V(D) \rightarrow \mathbb{R}^{+}$with $u \geq l$, and for any $S \subseteq V(D)$ with $|S| \leq s$ and with $u(S) \geq k \geq l(S)$, D has $k$ arc-disjoint spanning in-arborescences whose roots are in $S$ in such a way that every $r \in S$ is the root of at least $l(r)$ and at most $u(r)$ of such spanning arborescences.

For each $i \in\{1,2,3,4\}$, define

$$
f_{i}(n, k, s)=\min \left\{|A(D)|: D \in \mathcal{A}_{i}(k, s) \text { is strict with }|V(D)|=n\right\} .
$$

For a digraph $D$, let $D^{-}$denote the digraph obtained from $D$ by reversing the orientation of each arc of $D$. Then for any $r \in V(D)=V\left(D^{-}\right)$, an $r^{+}$-arborescence of $D$ is an $r^{-}$-arborescence of $D^{-}$. Therefore, $f_{1}(n, k, s)=f_{2}(n, k, s)$ and $f_{3}(n, k, s)=f_{4}(n, k, s)$. As an application of our main result in this section, we shall determine the values of these functions. The following theorem of Edmonds is very useful. Edmonds [27] proved Part (i) of Theorem 6.2.3, and Part (ii) of Theorem 6.2.3 follows immediately from Part (i) by applying Part (i) to $D^{-}$.

Theorem 6.2.3. (Edmonds [27]) Let $D$ be a digraph and $r \in V(D)$.

(i) Then $D$ has $k$-arc-disjoint spanning $r^{+}$-arborescences if and only if for any nonempty subset $S \subseteq V(D)-r, d_{D}^{-}(S) \geq k$.

(ii) Then $D$ has $k$-arc-disjoint spanning $r^{-}$-arborescences if and only if for any nonempty subset $S \subseteq V(D)-r, d_{D}^{+}(S) \geq k$.

Now we can state and prove our main result in this section.

Theorem 6.2.4. Let $k \geq s>0$ be integers. The following holds.

$$
\begin{aligned}
\mathcal{D}(k) & =\mathcal{A}_{1}(k, 1)=\mathcal{A}_{2}(k, 1)=\mathcal{A}_{1}(k, s)=\cdots= \\
& =\mathcal{A}_{2}(k, s)=\mathcal{A}_{3}(k, s)=\mathcal{A}_{4}(k, s) .
\end{aligned}
$$

Proof. We first observe that by Definition 6.2.2, we have $\mathcal{A}_{1}(k, s) \subseteq \mathcal{A}_{1}(k, 1)$ and $\mathcal{A}_{2}(k, s) \subseteq$ $\mathcal{A}_{2}(k, 1)$. By choosing $u=l$, we also have $\mathcal{A}_{3}(k, s) \subseteq \mathcal{A}_{1}(k, s)$ and $\mathcal{A}_{4}(k, s) \subseteq \mathcal{A}_{2}(k, s)$. It suffices to prove that $\mathcal{A}_{1}(k, 1) \subseteq \mathcal{A}_{3}(k, s), \mathcal{A}_{2}(k, 1) \subseteq \mathcal{A}_{4}(k, s)$, and $\mathcal{D}(k)=\mathcal{A}_{1}(k, 1)=\mathcal{A}_{2}(k, 1)$. Proof of $\mathcal{D}(k)=\mathcal{A}_{1}(k, 1)=\mathcal{A}_{2}(k, 1)$. For any $D \in \mathcal{D}(k)$, and for any $\emptyset \neq S \subseteq V(D)-r$, since $D \in \mathcal{D}(k)$, both $d_{D}^{-}(X) \geq k$ and $d_{D}^{+}(X) \geq k$, and so by Theorem 6.2 .3 , both $D \in \mathcal{A}_{1}(k, 1)$ and $D \in \mathcal{A}_{2}(k, 1)$. Hence $\mathcal{D}(k) \subseteq \mathcal{A}_{1}(k, 1) \cap \mathcal{A}_{2}(k, 1)$. 
Let $D \in \mathcal{A}_{1}(k, 1)$. For any $\emptyset \neq X \subset V(D)$, choose a vertex $r \in X$. Since $D \in \mathcal{A}_{1}(k, 1)$, $D$ has $k$-spanning $r^{+}$-arborescences. Since every spanning $r^{+}$-arborescences must have at least on arc in $\partial_{D}^{+}(X)$, we have $d_{D}^{+}(X) \geq k$. This implies $\mathcal{A}_{1}(k, 1) \subseteq \mathcal{D}(k)$. With a similar argument, we also have $\mathcal{A}_{2}(k, 1) \subseteq \mathcal{D}(k)$. This justifies $\mathcal{D}(k)=\mathcal{A}_{1}(k, 1)=\mathcal{A}_{2}(k, 1)$.

Proof of $\mathcal{A}_{1}(k, 1) \subseteq \mathcal{A}_{3}(k, s)$ and $\mathcal{A}_{2}(k, 1) \subseteq \mathcal{A}_{4}(k, s)$. By symmetry, it suffices to prove $\mathcal{A}_{1}(k, 1) \subseteq \mathcal{A}_{3}(k, s)$.

Let $D \in \mathcal{A}_{1}(k, 1)$. Suppose that a subset $S \subseteq V(D)$ with $|S| \leq s$, and functions $u, l: V(D) \rightarrow \mathbb{R}^{+}$ with $u \geq l$ and with $u(S) \geq k \geq l(S)$ are given. Let $S=\left\{x_{1}, x_{2}, \cdots, x_{s}\right\}$ and $K_{1}=k-l(S)$. Define $a_{1}=\min \left\{K_{1}, u\left(x_{1}\right)-l\left(x_{1}\right)\right\}$. Inductively, assume that $a_{1}, a_{2}, \cdots, a_{h}$ and $K_{1}, K_{2}, \cdots, K_{h}$ have been defined. If $h<s$, then define $K_{h+1}=K_{h}-a_{h}$ and $a_{h+1}=\min \left\{K_{h+1}, u\left(x_{h+1}\right)-\right.$ $\left.l\left(x_{h+1}\right)\right\}$. We claim the following.

$$
\left\{\begin{array}{l}
l\left(x_{i}\right) \leq l\left(x_{i}\right)+a_{i} \leq u\left(x_{i}\right), \text { for each } i \text { with } 1 \leq i \leq s, \\
\sum_{i=1}^{s}\left[l\left(x_{i}\right)+a_{i}\right]=k .
\end{array}\right.
$$

Since $u \geq l$, by the definition of the $a_{i}$ 's, we conclude that $a_{i} \geq 0$ and so $l\left(x_{i}\right) \leq l\left(x_{i}\right)+a_{i} \leq$ $l\left(x_{i}\right)+u\left(x_{i}\right)-l\left(x_{i}\right)=u\left(x_{i}\right)$. Let $t$ be the smallest integer with $1 \leq t \leq s$ such that $a_{t}=K_{t}$. If $t<s$, then by the definition of $K_{t^{\prime}}$ and $a_{t^{\prime}}$, for any $s \geq t^{\prime}>t$, we have $K_{t^{\prime}}=0$, and so $a_{t^{\prime}}=0$. Thus the second equality in (6.2) follows as shown below.

$$
\begin{aligned}
\sum_{i=1}^{s}\left[l\left(x_{i}\right)+a_{i}\right] & =l(S)+\sum_{i=1}^{t} a_{i}=l(S)+\sum_{i=1}^{t-1} a_{i}+K_{t} \\
& =l(S)+\sum_{i=1}^{t-1} a_{i}+\left(K_{t-1}-a_{t-1}\right) \\
& =l(S)+\sum_{i=1}^{t-2} a_{i}+\left(K_{t-2}-a_{t-2}\right)=\cdots= \\
& =l(S)+K_{1}=l(S)+(k-l(S))=k .
\end{aligned}
$$

We now construct a new graph $D^{\prime}$ from $D$ by adding a new vertex $r \notin V(D)$, such that for each $x_{i} \in S$, there is a set $A_{i}$ of exactly $l\left(x_{i}\right)+a_{i}$ parallel arcs from $r$ to $x_{i}$. By this definition and by (6.2), we have $d_{D^{\prime}}^{+}(r)=\sum_{i=1}^{s}\left[l\left(x_{i}\right)+a_{i}\right]=k$ and $d_{D^{\prime}}^{-}(r)=0$. Since $D \in \mathcal{A}_{1}(k, 1)$ and since $\mathcal{D}(k)=\mathcal{A}_{1}(k, 1)$, we have $D \in \mathcal{D}(k)$. For any $X \subseteq V(D)$, if $X \neq V(D)$, then

$$
d_{D^{\prime}}^{-}(X)=d_{D}^{-}(X)+\left|(\{r\}, X \cap S)_{D^{\prime}}\right|
$$

and so $d_{D^{\prime}}^{-}(X) \geq d_{D}^{-}(X)$. As $D \in \mathcal{D}(k)$, we have $d_{D^{\prime}}^{-}(X) \geq k$. If $X=V(D)$, then $d_{D^{\prime}}^{-}(V(D))=$ $d_{D^{\prime}}^{+}(r)=k$. It follows by Theorem 6.2 .3 that $D^{\prime}$ has $k$-arc-disjoint spanning $r$-arborescences $T_{1}, T_{2}, \cdots, T_{k}$. Since $d_{D^{\prime}}^{+}(r)=k$, every arc in $\partial_{D^{\prime}}^{+}(r)$ is in exactly one of these $T_{i}$ 's. Hence for 
each $x_{i} \in S$, there are exactly $l\left(x_{i}\right)+a_{i}$ of these $T_{i}$ 's, each of which contains one arc parallel to $\left(r, x_{i}\right)$ in $A_{i}$ as the only arc entering $x_{i}$ in the arborescence. It follows that by removing $r$ from these arborescences, we obtained exactly $l\left(x_{i}\right)+a_{i}$ arc-disjoint $x_{i}$-arborescences of $D$. Consequently, by (6.2), $D$ has $k$ arc-disjoint spanning out-arborescences whose roots are in $S$ in such a way that every $x_{i} \in S$ is the root of at least $l\left(x_{i}\right)$ and at most $u\left(x_{i}\right)$ of such spanning arborescences. As $S$ is arbitrarily, this implies that $D \in \mathcal{A}_{3}(k, s)$, and so $\mathcal{A}_{1}(k, 1) \subseteq \mathcal{A}_{3}(k, s)$. With a similar argument, we also have $\mathcal{A}_{2}(k, 1) \subseteq \mathcal{A}_{2}(k, s)$. This completes the proof of the theorem.

By Theorem 6.2.4, we can obtain the following digraph versions of Theorem 1.3.1. As shown in Theorem 6.2.4, we have $\mathcal{D}(k)=\mathcal{A}_{1}(k, 1)=\mathcal{A}_{2}(k, 1)$, which immediately justifies Corollary 6.2 .5

Corollary 6.2.5. Let $D$ be a digraph and let $k$ be a positive integer. The following are equivalent. (i) $\lambda(D) \geq k$.

(ii) For every vertex $v, D$ has $k$-arc-disjoint spanning $v^{+}$-arborescen-ces.

(iii) For every vertex $v$, $D$ has $k$-arc-disjoint spanning $v^{-}$-arboresce-nces.

Corollary 6.2.6. Let $n>k>0$ be integers, and $D$ be a strict digraph on $n$ vertices. Then $D \in \mathcal{D}(k)$ if and only if for any vertex $v \in V(D)$, there exists an arc subset $X$ with $|X| \leq k$ such that $D-X$ has $k$-arc-disjoint spanning $v^{+}$-arborescences.

Proof. Suppose $D \in \mathcal{D}(k)$. Then by Theorem $6.2 .4, \mathcal{D}(k)=\mathcal{A}_{1}(k, 1)$, and so for any vertex $v \in V(D), D$ has $k$-arc-disjoint spanning $v^{+}$-arborescences $T_{1}, T_{2}, \ldots, T_{k}$. By Theorem 6.2.9, $|A(D)| \geq n k$, and so $\left|A(D)-\cup_{i=1}^{k} E\left(T_{i}\right)\right| \geq k n-k(n-1)=k$. It follows that there exists a subset $X \subseteq A(D)-\cup_{i=1}^{k} E\left(T_{i}\right)$ with $|X|=k$, and so $D-X$ has $k$-arc-disjoint spanning $v^{+}$-arborescences.

Conversely, for any non empty proper subset $S \subset V(D)$ with $S \neq \emptyset$, pick a vertex $v \in S$. By assumption, there exists an arc subset $X \subset A(D)$ such that $D-X$ has $k$-arc-disjoint spanning arborescences. It follows that there must be at least $k$ arcs on these arborescences going from $S$ to $V(D)-S$, and so $\left|\partial_{D}^{+}(S)\right| \geq k$. By definition, $D \in \mathcal{D}(k)$. This proves the corollary.

To determine values of the functions defined in (6.1), we need former results of Walecki and of Tillson. In Theorems 6.2.7 and 6.2.8, $m \geq 1$ is an integer.

Theorem 6.2.7. (Waleski, see e.g. [13], [70] for the construction.) The edges of the complete graph on $2 m+1$ vertices can be decomposed into $m$ edge-disjoint Hamilton cycles.

Theorem 6.2.8. (Tillson [90]) For $2 m \geq 8$, the arcs of the complete digraph on $2 m$ vertices can be decomposed into $2 m-1$ arc-disjoint directed Hamilton cycles. 
Theorem 6.2.9. For each $i \in\{1,2,3,4\}$, and for any integers $k, n, s$ with $n \geq k+1$, and $n>s>0$,

$$
f_{i}(n, k, s)=n k
$$

Proof. Note that $\mathcal{A}(k)=\mathcal{A}_{1}(k, 1)$. Define

$$
F(n, k)=\min \{|A(D)|: D \in \mathcal{D}(k) \text { is strict with }|V(D)|=n\}
$$

By Theorem 6.2.4, for $1 \leq i \leq 4$, we have $F(n, k)=f_{i}(n, k, s)$. For any $D \in D(k)$ and for any $v \in V(D)$, we have $d_{D}^{+}(v) \geq k$, and so $|A(D)|=\sum_{v \in V(D)} d_{D}^{+}(v) \geq n k$. Hence $F(n, k) \geq n k$.

To show that $F(n, k) \leq n k$, for any $n$ and any $k \leq n-1$, we shall construct a strict digraph $D \in D(k)$ such that

$$
\text { for any } v \in V(D) \text {, we have } d_{D}^{+}(v)=d_{D}^{-}(v)=k \text {. }
$$

If such a digraph $D$ can be constructed, then $F(n, k) \leq|A(D)|=n k$, and so (6.3) is justified.

For each integer $n \geq 3$, let $K_{n}$ denote the complete (undirected) graph on $n$ vertices and $K_{n}^{*}$ denote the strict complete digraph on $n$ vertices. For each $n \geq 3$ with $n \notin\{4,6\}$ and for each $1 \leq k \leq n-1$, we shall construct a strict digraph $D(n, k)$ on $n$ vertices such that $D(n, k)$ has $k$-arc-disjoint directed Hamilton cycles. To do that, we show that $K_{n}^{*}$ has arc-disjoint directed Hamilton cycles $C_{1}, C_{2}, \cdots C_{n-1}$. Then we define $D(n, k)=K_{n}^{*}\left[E\left(C_{1}\right) \cup E\left(C_{2}\right) \cup \cdots \cup E\left(C_{k}\right)\right]$, for each $k$ with $1 \leq k \leq n-1$. Note that any Hamilton cycle $C$ of $K_{n}$ can be oriented into two arc-disjoint directed Hamilton cycles in $K_{n}^{*}$ with opposite direction to each other. When $n \in\{4,6\}$, we follow the similar idea to proceed the proof except when $k=n-1$. In the rest of the proofs of this theorem, if $H_{1}$ and $H_{2}$ are sub-digraphs of a digraph $D$, then $H_{1} \cup H_{2}$ denotes the sub-digraph induced by the arc set $E\left(H_{1}\right) \cup E\left(H_{2}\right)$. We will construct $D(n, k)$ satisfying (6.5) in each of the following cases.

Case 1: $n \in\{4,6\}$.

Assume that $n \in\{4,6\}$. Then by definition, $K_{n}^{*} \in \mathcal{D}(n-1)$ and $K_{n}^{*}$ satisfies (6.5) with $k=n-1$, and so we have both $D(4,3)$ and $D(6,5)$.

For $n=4$, let $C_{1}^{4}=(0,3,1,2)$ and $C_{2}^{4}=(0,2,1,3)$. Then $D(4,2)=C_{1}^{4} \cup C_{2}^{4}$ and $D(4,1)=C_{1}^{4}$. For $n=6$, let $C_{1}^{6}=(0,1,2,5,4,3), C_{2}^{6}=(0,5,1,3,2,4), C_{3}^{6}=(0,3,4,5,2,1)$ and $C_{4}^{6}=$ $(0,4,2,3,1,5)$. Then $D(6,4)=C_{1}^{6} \cup C_{2}^{6} \cup C_{3}^{6} \cup C_{4}^{6}, D(6,3)=C_{1}^{6} \cup C_{2}^{6} \cup C_{3}^{6}, D(6,2)=C_{1}^{6} \cup C_{2}^{6}$, and $D(6,1)=C_{1}^{6}$.

Case 2: $n=2 s+1$ and $s \geq 1$.

By Theorem 6.2.7, the edge set of $K_{n}$, the undirected complete graph on $n$ vertices, can be decomposed into $s$ edge-disjoint Hamilton cycles. Each of these Hamilton cycles can have two opposite orientations. These give rise to a decomposition of $E\left(K_{n}^{*}\right)$ into $n-1$ arc-disjoint 
directed Hamilton cycles $C_{1}^{n}, C_{2}^{n}, \cdots, C_{n-1}^{n}$. Define $D(n, k)=\bigcup_{i=1}^{k} C_{i}^{n}$, for $k=1,2, \cdots, n-1$. As each $C_{i}^{n}$ is a directed Hamilton cycle, $D(n, k)$ satisfies $(6.5)$.

Case 3: $n=2 s$ and $s \geq 4$.

By Theorem $6.2 .8, E\left(K_{n}^{*}\right)$ can be decomposed into $n-1$ directed Hamiltonian cycles $C_{1}^{n}, C_{2}^{n}$, $\cdots, C_{n-1}^{n}$. Define $D(n, k)=\bigcup_{i=1}^{k} C_{i}^{n}$, for $k=1,2, \cdots, n-1$. As each $C_{i}^{n}$ is a directed Hamilton cycle, $D(n, k)$ satisfies $(6.5)$.

As for each $n \geq 3$ and $k \leq n-1$, a digraph $D(n, k)$ satisfying (6.5) can be found, by (6.4), we have $F(n, k) \leq k n$. This proves $(6.3)$.

\subsection{Characterization of Uniformly Dense Digraphs}

For a graph $G$, we follow [11] to use $c(G)$ to denote the number of components of $G$. Then the strength $\eta(G)$ and fractional arboricity $\gamma(G)$ of $G$ are defined as

$$
\eta(G)=\min _{X \subseteq E(G)}\left\{\frac{|X|}{c(G-X)-c(G)}\right\}
$$

and

$$
\gamma(G)=\max _{X \subseteq E(G)}\left\{\frac{|X|}{V(G[X])-c(G[X])}\right\}
$$

where the minimum and maximum are taken over all subsets $X$ such that the corresponding denominators are not zero. In [15], it has been indicated that the well-known spanning tree packing theorem of Nash-Williams [77] and Tutte [91] can be restated as the following.

Theorem 6.3.1. A nontrivial graph $G$ has $k$ edge-disjoint spanning trees if and only if $\eta(G) \geq k$.

In [15], an attempt to obtain a fractional version of Theorem 1.3.2 was made, and the following characterization is proved.

Theorem 6.3.2. (Catlin et al. , Theorem 6 of [15]) Let $G$ be a connected graph. The following are equivalent.

(i) $\gamma(G)(|V(G)|-1)=|E(G)|$.

(ii) $\eta(G)(|V(G)|-1)=|E(G)|$.

(iii) $\eta(G)=\gamma(G)$.

(iv) For any integers $s \geq t>0$ with $\gamma(G)=\frac{s}{t}$, $G$ has s spanning trees $T_{1}, T_{2}, \cdots, T_{s}$ such that every edge e $\in E(G)$ is in exactly $t$ members in the multiset $\left\{T_{1}, T_{2}, \cdots, T_{s}\right\}$.

(v) For any integers $s \geq t>0$ with $\eta(G)=\frac{s}{t}$, $G$ has s spanning trees $T_{1}, T_{2}, \cdots, T_{s}$ such that every edge e $\in E(G)$ is in exactly $t$ members in the multiset $\left\{T_{1}, T_{2}, \cdots, T_{s}\right\}$.

Any graph $G$ satisfying Theorem 6.3.2(i) is called uniformly dense. See [15] and [48] for more background on uniformly dense graphs. The purpose of this section is to obtain the 
digraph version of Theorem 6.3.2. For a digraph $D$ with $n=|V(D)| \geq 2$, let $\mathcal{P}=\mathcal{P}(D)=$ $\left\{\left(X_{1}, X_{2}, \cdots, X_{m}\right) \mid\right.$ where $X_{1}, X_{2}, \cdots X_{p}$ are disjoint nonempty subsets of $\left.V(D)\right\}$. We define

$$
\eta(D)=\min _{\left(X_{1}, X_{2}, \cdots X_{p}\right) \in \mathcal{P}(D), p>1}\left\{\frac{\sum_{i=1}^{p}\left|\partial_{D}^{-}\left(X_{i}\right)\right|}{p-1}\right\}
$$

and

$$
\gamma(D)=\max _{\left(X_{1}, X_{2}, \cdots X_{p}\right) \in \mathcal{P}(D), p<n}\left\{\frac{|A(D)|-\sum_{i=1}^{p}\left|\partial_{D}^{-}\left(X_{i}\right)\right|}{n-p}\right\} .
$$

Frank proved two theorems analogues to the spanning tree packing theorem of Nash-Williams ([77]) and Tutte ([91]) and the forest covering theorem of Nash-William ([78]). A branching of a digraph $D$ is a sub digraph $B$ of $D$ such that every weakly connected component of $B$ is an arborescence.

Theorem 6.3.3. (Frank [36]) Let D be a nontrivial digraph. The following are equivalent.

(i) $D$ is has $k$-arc-disjoint spanning arborescences.

(ii) $\eta(D) \geq k$.

Theorem 6.3.4. (Frank [36]) Let D be a nontrivial digraph. The following are equivalent.

(i) D has $k$ branchings such that every arc of $D$ is in at least one of them.

(ii) $\gamma(D) \leq k$.

Following Theorem 6.3.2, it is natural to define a digraph as uniformly dense if $\eta(D)=\gamma(D)$. We will apply Theorems 6.3.3 and 6.3.4 to obtain the characterization of uniformly dene digraph of Theorem 6.3.2, which justifies the definition of uniformly dense digraphs. We need two lemmas.

Lemma 6.3.5. Let $D$ be a digraph, and $k, t>0$ be integers.

(i) $D$ has $k$ spanning arborescences such that every $e \in A(D)$ lies in at most $t$ of them if and only if $\eta(D) \geq \frac{k}{t}$.

(ii) $D$ has $k$ branchings such that every $e \in A(D)$ lies in at least $t$ of them if and only if $\gamma(D) \leq \frac{k}{t}$

Proof. Let $D_{t}$ be the digraph obtained from $D$ by replacing each ar $e \in A(D)$ by $t$ parallel $\operatorname{arcs}\left\{e^{1}, e^{2}, \cdots, e^{t}\right\}$, each having the same head and tails as $e$.

Then $D_{t}$ has $k$ arc-disjoint spanning arborescences if and only if $D$ has $k$ spanning arborescences such that every $e \in A(D)$ lies in at most $t$ of them. Moreover, for each $X \subseteq(D)$, we have

$$
t\left|\partial_{D}^{-}(X)\right|=\left|\partial_{D_{t}}^{-}(X)\right|
$$

By Theorem 6.3.3, $G$ has $k$ branchings such that every $e \in A(D)$ lies in at least $t$ of them if and only if $\eta\left(D_{t}\right) \geq k$. By (6.6), $\eta\left(D_{t}\right) \geq k$ if and only if for any $\left(X_{1}, X_{2}, \ldots, X_{p}\right) \in \mathcal{P}(D)$ with $p>1$,

$$
k \leq \eta\left(D_{t}\right)=\frac{\sum_{i=1}^{p}\left|\partial_{D_{t}}^{-}\left(X_{i}\right)\right|}{p-1}=\frac{\sum_{i=1}^{p} t\left|\partial_{D}^{-}\left(X_{i}\right)\right|}{p-1} .
$$

Thus $\eta(D) \geq \frac{k}{t}$, and so (i) holds. The proof for (ii) is similar and is omitted. 
Lemma 6.3.6. Let $D$ be a digraph on $|V(D)|>1$ vertices. Define $d(D)=\frac{|A(D)|}{|V(D)|-1}$, Then

$$
\eta(D) \leq d(D) \leq \gamma(D)
$$

Proof. Choose $p=|V(D)|>1$ and $X_{i}=\left\{v_{i}\right\}$ in (6.6), to get the first inequality. Then choose $p=1$ and $X_{1}=V(D)$ in (6.7) to have the second.

Theorem 6.3.7. Let $D$ be a weakly connected nontrivial digraph on $n$ vertices. The followings are equivalent. (A digraph satisfying any one of the following is called a uniformly dense digraph).

(i) $|A(D)|=\eta(D)(n-1)$.

(ii) $|A(D)|=\gamma(D)(n-1)$.

(iii) $\eta(D)=\gamma(D)$.

(iv) For any integers $s \geq t>0$ such that $\gamma(D)=\frac{s}{t}$. there exists an integer $t>0$ such that $D$ has a family $\mathcal{F}=\left\{T_{1}, T_{2}, \cdots, T_{s}\right\}$ of spanning arborescences such that every $e \in A(D)$ lies in exactly $t$ members in $\mathcal{F}$.

(v) For any integers $s \geq t>0$ such that $\eta(D)=\frac{s}{t}$. there exists an integer $t>0$ such that $D$ has a family $\mathcal{F}=\left\{T_{1}, T_{2}, \cdots, T_{s}\right\}$ of spanning arborescences such that every $e \in A(D)$ lies in exactly $t$ members in $\mathcal{F}$.

Proof. By Lemma 6.3.6, (iii) implies (i) and (ii). It remains to show that each of (i) and (ii) implies (iv), and (iv) implies (iii).

(i) $\Longrightarrow$ (iv). (The proof for (ii) $\Longrightarrow$ (iv) is similar, and will be omitted.) For any integers $s \geq t>0$ with $s=t \eta(D)$, by Lemma 6.3.5 (i). $D$ has a family $\mathcal{F}$ of $s$ spanning arborescences such that every arc in $D$ lies in at most $t$ members of $\mathcal{F}$. It follows by (i) that

$$
t(\eta(D)(n-1)=s(n-1) \leq t|A(D)| \leq t \eta(D)(n-1) .
$$

This forces that every arc of $D$ is in exactly $t$ members of $\mathcal{F}$.

(iv) $\Longrightarrow$ (iii). Suppose that $D$ has a family $\mathcal{F}$ of $s$ spanning arborescences with the property that every $e \in A(D)$ lies in exactly $t$ members in $\mathcal{F}$. Then by Lemma 6.3 .5 and by Lemma 6.3.6,

$$
\gamma(D) \leq \frac{s}{t} \leq \eta(D) \leq \gamma(D)
$$

Hence we must have (iii). 


\section{Chapter 7}

\section{On the Lower Bound of $k$-maximal Digraphs}

\subsection{The Problem}

If $G$ is a simple graph, then $G^{c}$ denotes the complement of $G$. If $X \subseteq E\left(G^{c}\right)$, then $G+X$ is the simple graph with vertex set $V(G)$ and edge set $E(G) \cup X$. We will use $G+e$ for $G+\{e\}$. Likewise, if $D$ is a simple digraph, let $D^{c}$ denote the complement of $D$. For $X \subseteq A\left(D^{c}\right)$ and $e \in A\left(D^{c}\right)$, we similarly define the simple digraphs $D+X$ and $D+e$, respectively. If $H, K$ are subdigraphs of $D$, then $H \cup K$ is the subdigraph of $D$ with vertex set $V(H) \cup V(K)$ and arc set $A(H) \cup A(K)$. Throughout this chapter, we use the notation $(u, v)$ to denote an arc oriented from $u$ to $v$ in a digraph. If $W \subseteq V(D)$ or if $W \subseteq A(D)$, then $D[W]$ denotes the subdigraph of $D$ induced by $W$. For $v \in V(D)$, we use $D-v$ for $D[V(D)-\{v\}]$. For graphs $H$ and $G$, we denote $H \subseteq G$ when $H$ is a subgraph of $G$. Similarly, for digraphs $H$ and $D, H \subseteq D$ means $H$ is a subdigraph of $D$. We write $D \cong D^{\prime}$ to represent the fact that $D$ and $D^{\prime}$ are isomorphic digraphs.

Given a graph $G$, Matula $[73,74,75]$ first studied the quantity

$$
\bar{\kappa}^{\prime}(G)=\max \left\{\kappa^{\prime}(H): H \subseteq G\right\} .
$$

He called $\bar{\kappa}^{\prime}(G)$ the strength of $G$. Mader [71] considered an extremal problem related to $\bar{\kappa}^{\prime}(G)$. For an integer $k>0$, a simple graph $G$ with $|V(G)| \geq k+1$ is $k$-maximal if $\bar{\kappa}^{\prime}(G) \leq k$ but for any edge $e \in E\left(G^{c}\right), \bar{\kappa}^{\prime}(G+e)>k$. In [71], Mader proved the following.

Theorem 7.1.1. (Mader [71]) If $G$ is a k-maximal graph on $n>k \geq 1$ vertices, then

$$
|E(G)| \leq(n-k) k+\left(\begin{array}{c}
k \\
2
\end{array}\right) .
$$

Furthermore, this bound is best possible. 
It has been noted that being a $k$-maximal graph requires a certain level of edge density. Towards this direction, the following was proved in 1990.

Theorem 7.1.2. (Lai, Theorem 2 of [53]) If $G$ is a $k$-maximal graph on $n>k+1 \geq 2$ vertices, then

$$
|E(G)| \geq(n-1) k-\left(\begin{array}{c}
k \\
2
\end{array}\right)\left\lfloor\frac{n}{k+2}\right\rfloor .
$$

Furthermore, this bound is best possible.

It is natural to consider extending the theorems above to digraphs. Towards this direction, for a digraph $D$, we define

$$
\bar{\lambda}(D)=\max \{\lambda(H): H \subseteq D\} .
$$

Let $k \geq 0$ be an integer. A simple digraph $D$ with $|V(D)| \geq k+1$ is $k$-maximal if $\bar{\lambda}(D) \leq k$ but for any arc $e \in A\left(D^{c}\right), \bar{\lambda}(D+e) \geq k+1$. Following Matula [73], we may also call $\bar{\lambda}(D)$ the strength of digraph $D$ and so a $k$-maximal digraph is also called a $k$-maximal strength digraph. For positive integers $n$ and $k$ satisfying $n \geq k+1$, define

$$
\mathcal{D}(n, k)=\{D: \quad D \text { is a simple digraph with }|V(D)|=n \text { and } D \text { is } k \text {-maximal }\} .
$$

Thus we are to investigate the upper and lower bounds of the set of numbers $\{|A(D)|: D \in$ $\mathcal{D}(n, k)\}$. For notational convenience, if $h<k$, we define $\left(\begin{array}{c}h \\ k\end{array}\right)=0$. The following has been obtained.

Theorem 7.1.3. (Anderson et al. , Theorem 1.2 of [6], see also [5]) Let $n$ and $k$ be positive integers with $n \geq k+1$. If $D \in \mathcal{D}(n, k)$, then

$$
|A(D)| \leq k(2 n-k-1)+\left(\begin{array}{c}
n-k \\
2
\end{array}\right) .
$$

Furthermore, the bound is best possible.

In fact, all extremal digraphs in $\mathcal{D}(n, k)$ reaching this upper bound are characterized in [6]. The purpose of this research is to determine the lower bound. The following is the main result.

Theorem 7.1.4. Let $n$ and $k$ be positive integers with $n \geq k+1$. If $D \in \mathcal{D}(n, k)$, then

$$
|A(D)| \geq\left(\begin{array}{l}
n \\
2
\end{array}\right)+(n-1) k+\left\lfloor\frac{n}{k+2}\right\rfloor\left(1+2 k-\left(\begin{array}{c}
k+2 \\
2
\end{array}\right)\right) .
$$

Furthermore, the bound is best possible.

In the next section, we investigate properties of $k$-maximal digraphs. In Section 7.3, we present a constructive characterization of a family of $k$-maximal digraphs $\mathcal{E}^{\prime}(k)$. In the last section, we will prove Theorem 7.1.4 and show that the members in the family $\mathcal{E}^{\prime}(k)$ are precisely the digraphs attaining the upper bound in Theorem 7.1.4. 


\subsection{Properties of $k$-maximal Digraphs}

Throughout this section, $n$ and $k$ denote integers with $n>k \geq 0$. We present some properties of $k$-maximal digraphs to be utilized later. Let $\mathcal{D}(k)$ be the family of all $k$-maximal digraphs. Thus

$$
\mathcal{D}(k)=\cup_{n \geq k+1} \mathcal{D}(n, k) .
$$

For any integer $n \geq 0$, let $K_{n}^{*}$ denote the complete digraph on $n$ vertices. Thus $K_{n}^{*}$ is a simple digraph such that for any pair of distinct vertices $u, v \in V\left(K_{n}^{*}\right)$, both $(u, v)$ and $(v, u)$ are in $A\left(K_{n}^{*}\right)$. By definition, we observe the following

$$
K_{k+1}^{*} \in \mathcal{D}(k) \text { and if } H \in \mathcal{D}(k) \text { and }|V(H)|=k+1 \text {, then } H \cong K_{k+1}^{*} \text {. }
$$

Lemma 7.2.1. (Anderson et al. , Lemma 2.1 of [6]) $A$ digraph $D \in \mathcal{D}(0)$ if and only if $D$ is an acyclic tournament.

Lemma 7.2.1 indicates that we may exclude the case $k=0$ in our study. Therefore, we will always assume that $k>0$ in the rest of this chapter. Following [9], if $D$ is a digraph and if $X, Y \subseteq V(D)$, then define

$$
(X, Y)_{D}=\{(x, y) \in A(D): x \in X, y \in Y\}
$$

We further define that, for $X \subseteq V(D)$,

$$
\partial_{D}^{+}(X)=(X, V(D)-X)_{D} \text { and } \partial_{D}^{-}(X)=(V(D)-X, X)_{D}
$$

For each $v \in V(D)$, we define

$$
N_{D}^{+}(v)=\{u \in V(D):(v, u) \in A(D)\} \text { and } N_{D}^{-}(v)=\{u \in V(D):(u, v) \in A(D)\} .
$$

When the digraph $D$ is understood from the context, we sometimes omit the subscript $D$ in the notations above. By the definition of arc-strong connectivity in [9], a digraph $D$ satisfies $\lambda(D) \geq k$ if and only if for any nonempty proper subset $X \subset V(D),\left|\partial_{D}^{+}(X)\right| \geq k$.

Definition 7.2.2. Let $H \in \mathcal{D}(k)$ and let $\left\{v_{1}, v_{2}, \cdots, v_{k}\right\} \subset V(H)$ be a subset of $k$ distinct vertices. Let $u$ be a vertex not in $V(H)$. Define a digraph $\left[H, K_{1}\right]_{k}\left(\left[K_{1}, H\right]_{k}\right.$, respectively) as follows:

(i) $V\left(\left[H, K_{1}\right]_{k}\right)=V\left(\left[K_{1}, H\right]_{k}\right)=V(H) \cup\{u\}$.

(ii) $A\left(\left[H, K_{1}\right]_{k}\right)=A(H) \cup\left\{\left(v_{1}, u\right),\left(v_{2}, u\right), \cdots,\left(v_{k}, u\right)\right\} \cup\left(\bigcup_{v \in V(H)}\{(u, v)\}\right) \cdot\left(A\left(\left[K_{1}, H\right]_{k}\right)=\right.$ $A(H) \cup\left\{\left(u, v_{1}\right),\left(u, v_{2}\right), \cdots,\left(u, v_{k}\right)\right\} \cup\left(\bigcup_{v \in V(H)}\{(v, u)\}\right)$, respectively).

Note that each of $\left[H, K_{1}\right]_{k}$ and $\left[K_{1}, H\right]_{k}$ represents a family of graphs as the set $\left\{v_{1}, v_{2}, \cdots, v_{k}\right\} \subset$ $V(H)$ may vary. 
Definition 7.2.3. Let $H_{1}, H_{2} \in \mathcal{D}(k)$, and let $\left\{u_{1}, u_{2}, \cdots, u_{k}\right\} \subset V\left(H_{1}\right)$ be a multiset of $V\left(H_{1}\right)$ and $\left\{v_{1}, v_{2}, \cdots, v_{k}\right\} \subset V\left(H_{2}\right)$ be a multiset of $V\left(H_{2}\right)$ such that all the arcs $\left(u_{1}, v_{1}\right),\left(u_{2}, v_{2}\right), \cdots$, $\left(u_{k}, v_{k}\right)$ are distinct. Define a digraph $\left[H_{1}, H_{2}\right]_{k}$ as follows.

(i) $V\left(\left[H_{1}, H_{2}\right]_{k}\right)=V\left(H_{1}\right) \cup V\left(H_{2}\right)$.

(ii) $A\left(\left[H_{1}, H_{2}\right]_{k}\right)=A\left(H_{1}\right) \cup A\left(H_{2}\right) \cup\left\{\left(u_{1}, v_{1}\right),\left(u_{2}, v_{2}\right), \cdots,\left(u_{k}, v_{k}\right)\right\} \cup\left(\bigcup_{u \in V\left(H_{1}\right), v \in V\left(H_{2}\right)}\{(v, u)\}\right)$.

Note that $\left[H_{1}, H_{2}\right]_{k}$ represents a family of digraphs.

Lemma 7.2.4. (Anderson et al. , Corollary 2.6 of [6]) Let $D \in \mathcal{D}(k)-\left\{K_{k+1}^{*}\right\}$ be a digraph.

Then there exists a nonempty proper subset $X \subseteq V(D)$ such that one of the following holds.

(i) $|X|=1$, and for some $H \in \mathcal{D}(k), D \in\left[K_{1}, H\right]_{k}$.

(ii) $|V(D)-X|=1$ and for some $H \in \mathcal{D}(k), D \in\left[H, K_{1}\right]_{k}$.

(iii) For some $H_{1}, H_{2} \in \mathcal{D}(k)$, we have $D[X]=H_{1}$ and $D \in\left[H_{1}, H_{2}\right]_{k}$.

\subsection{Structure of $k$-maximal Digraphs}

Let $H(k, 2)$ be the digraph obtained from $K_{k+2}^{*}$ by removing an arc from $K_{k+2}^{*}$. Note that if $D \cong H(k, 2)$, then $D$ has exactly one vertex (to be denoted $x^{-}(D)$ ) of indegree $k$ and exactly one vertex (to be denoted $x^{+}(D)$ ) of outdegree $k$.

Definition 7.3.1. Let $n$ and $k$ be positive integers. Define $\mathcal{S}(n, k)$ to be the set of all integral sequences $\left(s_{1}, s_{2}, \cdots, s_{m}\right)$ satisfying $s_{1}+s_{2}+\ldots+s_{m}=n$ such that $s_{1}=k+2$, and for $i \geq 2, s_{i} \in\{1,-1, k+2,-(k+2)\}$. For any $\mathbf{s}=\left(s_{1}, s_{2}, \cdots, s_{m}\right) \in \mathcal{S}(n, k)$, define digraphs $L(\mathbf{s})=L\left(s_{1} s_{2}, \cdots, s_{m}\right)$ as follows.

(i) For $i=1$, then define $L_{1} \cong H(k, 2)$.

(ii-A) For $i \geq 2$, if $s_{i}=1$ ( $s_{i}=-1$, respectively), then define $L_{i} \in\left[L_{i-1}, K_{1}\right]_{k}\left(L_{i} \in\left[K_{1}, L_{i-1}\right]_{k}\right.$, respectively).

(ii-B) For $i \geq 2$, if $s_{i}=k+2\left(s_{i}=-(k+2)\right.$, respectively), then define $L_{i} \in\left[L_{i-1}, H(k, 2)\right]_{k}$ $\left(L_{i} \in\left[H(k, 2), L_{i-1}\right]_{k}\right.$, respectively), in such a way that for any $1 \leq t \leq i$ with $\left|s_{t}\right|=k+2$ we have $d_{L_{i}}^{+}\left(x^{+}\left(J_{t}\right)\right) \geq k+1\left(d_{L_{i}}^{-}\left(x^{-}\left(J_{t}\right)\right) \geq k+1\right.$, respectively).

(iii) Define $L(\mathbf{s})=L_{m}$. By Definitions 7.2.2 and 7.2.3, each $L(\mathbf{s})$ represents a collection of digraphs.

(iv) Given $\mathbf{s}=\left(s_{1}, s_{2}, \cdots, s_{m}\right) \in \mathcal{S}(n, k)$, define $J_{i}=K_{1}$ if $\left|s_{i}\right|=1$ and $J_{i}=H(k, 2)$ if $\left|s_{i}\right|=k+2$. Then the sequence of digraphs $J_{1}, J_{2}, \cdots, J_{m}$ is called a construction sequence of $L(\mathbf{s})$.

(v) Define $\mathcal{E}(n, k)=\{L(\mathbf{s}): \mathbf{s} \in \mathcal{S}(n, k)\}$ and $\left.\mathcal{E}(k)=\cup_{n \geq k+2}\left(\mathcal{E}(n, k) \cup\left\{K_{k+1}^{*}\right\}\right)\right\}$.

For a digraph $D$, an arc subset $W=(X, V(D)-X)_{D}$ for some proper non empty subset $X$ is called an arc-cut. If $|W|=t$ and $W$ is an arc-cut, then $W$ is called a $t$-arc-cut. 
Observation 2. We will make a few observations from Definition 7.3.1.

(i) By definition, $H(k, 2) \in\{L(\mathbf{s})\}$ with $\mathbf{s}$ being the sequence of only one term $k+2$. Since there is only one arc $a \in A\left(H(k, 2)^{c}\right)$, we have $H(k, 2)+a=K_{k+2}^{*}$ and so

$$
H(k, 2) \in \mathcal{D}(k)
$$

(ii) Let $D \in \mathcal{E}(k)-\{H(k, 2)\}$. We may assume that $n=|V(D)|>k+2$ and for some $\mathbf{s}=\left(s_{1}, s_{2}, \ldots, s_{m}\right) \in(n, k), D \in L(\mathbf{s})$ with construction sequence $J_{1}, J_{2}, \cdots, J_{m}$. Using the notation in Definition 7.3.1, we let $L_{i}=D\left[\cup_{j+1}^{i} V\left(J_{j}\right)\right]$. For any $k$-arc-cut $W=(X, V(D)-$ $X)_{D}$ of $D$, there must be and $i$ with $1 \leq i<m$ such that $W=\left(V\left(L_{i}\right), V\left(J_{i+1}\right)\right)_{D}$ or $W=$ $\left(V\left(J_{i+1}\right), V\left(L_{i}\right)\right)_{D}$.

We will justify Observation 2(ii). Since $n=|V(D)|>k+2$, we have $m \geq 2$. When $m=2$, by Definition 7.3.1 (ii-A) and (ii-B), we observe that if $W_{1}$ is a $k$-arc-cut of $D$, then we must have $W_{1}=\left(V\left(J_{1}\right), V\left(J_{2}\right)\right)_{D}$ or $W_{1}=\left(V\left(J_{2}\right), V\left(J_{1}\right)\right)_{D}$. Hence we assume that $m>2$. Inductively, assume that for any digraph $D^{\prime} \in \mathcal{E}(k)-\{H(k, 2)\}$ with $\left|V\left(D^{\prime}\right)\right|<|V(D)|$ and with construction sequence $J_{1}^{\prime}, J_{2}^{\prime}, \ldots, J_{m^{\prime}}^{\prime}$, if $W^{\prime}$ is a $k$-arc-cut of $D^{\prime}$, then there must be an $i$ with $1 \leq i<m^{\prime}$ such that $W^{\prime}=\left(\cup_{j+1}^{i} V\left(J_{j}^{\prime}\right), V\left(J_{i+1}^{\prime}\right)\right)_{D^{\prime}}$ or $W^{\prime}=\left(V\left(J_{i+1}^{\prime}\right), \cup_{j+1}^{i} V\left(J_{j}^{\prime}\right)\right)_{D^{\prime}}$. Let $W=(X, V(D)-X)_{D}$ be an $k$-arc-cut of $D$. If $X \cap V\left(J_{m}\right)=\emptyset$ or if $J_{m} \subseteq X$, then by Definition $2, W$ is an $k$-arc-cut of $L_{m-1}$, and so by induction, there must be an $i$ with $1 \leq i<m-1$ such that Observation 2(ii) holds. Hence we must have $X \cap V\left(J_{m}\right) \neq \emptyset$ and $(V(D)-X) \cap J_{m} \neq \emptyset$. In this case, as $\left|V\left(J_{m}\right)\right| \geq 2$, we must have $s_{m}=k+2$ and $J_{m}=H(k, 2)$. It follows that $H(k, 2)$ contains an arc-cut $X \cap A\left(J_{m}\right)$ of size at most $k$. But by Definition 7.3.1(ii-B), $J_{m}$ does not have an arc-cut of size $k$. This contradiction justifies Observation 2(ii).

Observation 2(i) can be extended, as shown in Theorem 7.3.5 below.

Lemma 7.3.2. For any $D \in \mathcal{E}(k)$, we have

$$
\lambda(D)=\bar{\lambda}(D)=k
$$

Proof. By Definition 7.3.1, it suffices to show that if $D=L(\mathbf{s})$ for some $\mathbf{s}=\left(s_{1}, s_{2}, \cdots, s_{m}\right) \in$ $\mathcal{S}(n, k)$, then (7.3) holds. We argue by induction on $m$. By (7.2), (7.3) holds for $m=1$. Assume that $m>1$ and (7.3) holds for smaller values of $m$. We adopt the notation in Definition 7.3.1 and let $J_{1}, J_{2}, \cdots, J_{m}$ be the construction sequence of $D$. Let $\mathbf{s}^{\prime}=\left(s_{1}, s_{2}, \cdots, s_{m-1}\right)$ and $D^{\prime}=D-V\left(J_{m}\right)$. Then $\mathbf{s}^{\prime} \in \mathcal{S}\left(n-s_{m}, k\right)$, and $D^{\prime}=L\left(\mathbf{s}^{\prime}\right)$. By Definition 7.3.1, $D \in\left[D^{\prime}, J_{m}\right]_{k}$. By induction, $\lambda\left(D^{\prime}\right)=\bar{\lambda}\left(D^{\prime}\right)=k$.

We argue by contradiction to prove that $\lambda(D) \geq k$, and assume that $D$ has a proper nonempty subset $X \subset V(D)$ such that $\left|\partial_{D}^{+}(X)\right|<k$. If both $X \cap V\left(D^{\prime}\right) \neq \emptyset$ and $V\left(D^{\prime}\right)-X \neq \emptyset$, then by $\lambda\left(D^{\prime}\right)=k$, we have a contradiction $k>\left|\partial_{D}^{+}(X)\right| \geq\left|\left(V\left(D^{\prime}\right) \cap X, V\left(D^{\prime}\right)-X\right)_{D^{\prime}}\right| \geq$ $k$. Hence either $V\left(D^{\prime}\right) \cap X=\emptyset$ or $V\left(D^{\prime}\right) \subseteq X$. Similarly, as $J_{m} \in\left\{K_{1}, H(k, 2)\right\}$, if both 
$X \cap V\left(J_{m}\right) \neq \emptyset$ and $V\left(J_{m}\right)-X \neq \emptyset$, then $J_{m}=H(k, 2)$, and so $k>\left|\partial_{D}^{+}(X)\right| \geq \mid\left(V\left(J_{m}\right) \cap\right.$ $\left.X, V\left(J_{m}\right)-X\right)_{D^{\prime}} \mid \geq \lambda(H(k, 2))=k$, a contradiction. It follows that we must have $X=V\left(D^{\prime}\right)$ or $X=V\left(J_{m}\right)$. By Definition 7.2 .2 or 7.2 .3 , we have again a contradiction: $k>\left|\partial_{D}^{+}(X)\right| \geq$ $\min \left\{\left|\left(V\left(J_{m}\right), V\left(D^{\prime}\right)\right)_{D}\right|,\left|\left(V\left(D^{\prime}\right), V\left(J_{m}\right)\right)_{D}\right| \geq k\right.$. This proves that $\lambda(D) \geq k$.

We now prove $\bar{\lambda}(D)=k$ by contradiction. Assume that $D$ has a subdigraph $H$ such that $\lambda(H) \geq k+1$. If both $V(H) \cap V\left(D^{\prime}\right) \neq \emptyset$ and $V(H) \cap V\left(J_{m}\right) \neq \emptyset$, then $\lambda(H) \leq \mid(V(H) \cap$ $\left.V\left(D^{\prime}\right), V(H) \cap V\left(J_{m}\right)\right)_{H}|\leq|\left(V\left(D^{\prime}\right), V\left(J_{m}\right)\right)_{D} \mid=k$, contrary to $\lambda(H) \geq k+1$. Thus since $J_{m} \in\left\{K_{1}, H(k, 2)\right\}$, we must have $H \subseteq D^{\prime}$. By induction, $\bar{\lambda}\left(D^{\prime}\right)=k$, and so $\lambda(H) \leq \bar{\lambda}\left(D^{\prime}\right)=k$, contrary to the assumption $\lambda(H) \geq k+1$. This proves the lemma.

A special class of graphs in $\mathcal{E}(k)$ has been studied in [6]. Let $\mathcal{S}_{M}(n, k)$ be the subset of $\mathcal{S}(n, k)$ such that $\mathbf{s}=\left(s_{1}, s_{2}, \cdots, s_{m}\right) \in \mathcal{S}_{M}(n, k)$ if and only if $\left|s_{2}\right|=\left|s_{3}\right|=\cdots\left|s_{m}\right|=1$. Let $\mathcal{M}(k)=\cup_{n \geq k+2}\left\{L(\mathbf{s}): \mathbf{s} \in \mathcal{S}_{M}(n, k)\right\}$.

Theorem 7.3.3. (Anderson et al., Theorem 3.2(ii) of [6]) $\mathcal{M}(k) \subseteq \mathcal{D}(k)$.

The observations stated in Lemma 7.3.4 below follow immediately from Definition 7.3.1. For example, in Lemma 7.3.4(i), if for some $2 \leq t \leq m-1$, (7.4) holds, then the digraph sequence $J_{1}, J_{2}, \cdots, J_{t-1}, J_{t+1}, \cdots, J_{m}, J_{t}$ is also a construction sequence of $D$ such that for $\mathbf{s}^{\prime}=\left(s_{1}, \cdots, s_{t-1}, s_{t+1}, \cdots, s_{m}, s_{t}\right)$, we have then $D \in L\left(\mathbf{s}^{\prime}\right)$. The justification of Lemma 7.3.4(ii) is similar and will be omitted.

Lemma 7.3.4. Let $D \in L(\mathbf{s})$ for some $\mathbf{s}=\left(s_{1}, s_{2}, \cdots, s_{m}\right) \in \mathcal{S}(n, k)$ with a construction sequence $J_{1}, J_{2}, \cdots, J_{m}$. Each of the following holds.

(i) If for some $t$ with $2 \leq t \leq m-1$, and for all $j$ with $t+1 \leq j \leq m$,

$$
\text { either } s_{j}>0 \text { and }\left(J_{t}, J_{j}\right)_{D}=\emptyset \text {, or } s_{j}<0 \text { and }\left(J_{j}, J_{t}\right)_{D}=\emptyset,
$$

then $D-V\left(J_{t}\right)=L\left(\mathbf{s}^{\prime}\right)$, where $\mathbf{s}^{\prime}=\left(s_{1}, \cdots, s_{t-1}, s_{t+1}, \cdots, s_{m}\right) \in \mathcal{S}\left(n-s_{t}, k\right)$ and $D-V\left(J_{t}\right)=$ $L\left(\mathbf{s}^{\prime}\right)$.

(ii) Suppose that for some $t$ with $1 \leq t<m$, we have $s_{t+1}=k+2$. If for each $j$ with $t+2 \leq j \leq m$,

either $s_{j}>0$ and $\left(J_{1} \cup J_{2} \cup \cdots \cup J_{t}, J_{j}\right)_{D}=\emptyset$, or $s_{j}<0$ and $\left(J_{j}, J_{1} \cup J_{2} \cup \cdots \cup J_{t}\right)_{D}=\emptyset$,

then $D-V\left(J_{1} \cup J_{2} \cup \cdots \cup J_{t}\right)=L\left(\mathbf{s}^{\prime}\right)$, where $\mathbf{s}^{\prime}=\left(s_{t+1}, s_{t+2}, \cdots, s_{m}\right) \in \mathcal{S}\left(n-\sum_{i=1}^{t} s_{i}, k\right)$.

Lemma 7.3.4 can be applied in inductive augments involving digraphs in $\mathcal{E}(k)$. This allows us to prove a generalization of Theorem 7.3.3, as stated in the theorem below.

Theorem 7.3.5. Let $k \geq 1$ be an integer. Then $\mathcal{E}(k) \subseteq \mathcal{D}(k)$. 
Proof. Let $D \in \mathcal{E}(k)$ with $n=|V(D)|$. In the proof arguments below, we shall adopt the notation in Definition 7.3.1 to use $L_{1}, L_{2}, \cdots, L_{m}$ to denote the graphs in the process to build $L_{m}$.

We argue by induction on $n$ to prove the theorem. By Definition 7.3.1, $n \geq k+2$, and $n=k+2$ if and only if $D=H(k, 2)$. By $(7.2), D=H(k, 2) \in \mathcal{D}(k)$. Thus we may assume that $n>k+2$ and for any digraph $D^{\prime} \in \mathcal{E}(k)$ with $\left|V\left(D^{\prime}\right)\right| \leq n-1, D^{\prime} \in \mathcal{D}(k)$. We are to show that if $D \in \mathcal{E}(n, k)$, then $D \in \mathcal{D}(k)$.

By contradiction, we assume that $D \in \mathcal{E}(n, k)-\mathcal{D}(k)$, and so for some $a=(u, v) \in A\left(D^{c}\right)$, we have

$$
\bar{\lambda}(D+a) \leq k
$$

Assume that $D=L(\mathbf{s})$ for some $\mathbf{s}=\left(s_{1}, s_{2}, \cdots, s_{m}\right) \in \mathcal{S}(n, k)$ with $m$ minimized and $a=$ $(u, v) \in A\left(D^{c}\right)$; and let $J_{1}, J_{2}, \cdots, J_{m}$ be the corresponding construction sequence of $D$. Since $n>k+2$, we have $m \geq 2$. By symmetry, we assume that $D \in\left[L_{m-1}, J_{m}\right]_{k}$. By induction, $L_{m-1} \in \mathcal{D}(k)$. If $u, v \in V\left(L_{m-1}\right)$, then $\bar{\lambda}(D+a) \geq \bar{\lambda}\left(L_{m-1}+a\right) \geq k+1$. Hence we may assume that

$$
u \in V\left(L_{m-1}\right) \text { and } v \in V\left(J_{m}\right)
$$

By (7.6),

there exists a nonempty proper subset $X \subset V(D+a)$, such that $\left|\partial_{D+a}^{+}(X)\right| \leq k$.

By Definition 7.3.1(ii) or (iii), there are $k$ arcs from $L_{m-1}$ to $J_{m}$. We assume that $\left(V\left(L_{m-1}\right)\right.$, $\left.V\left(J_{m}\right)\right)_{D}=\left\{a_{1}, a_{2}, \cdots, a_{k}\right\}$. Let $a_{i}=\left(v_{i}, w_{i}\right), 1 \leq i \leq k$. By Definition 7.3.1, $\left\{v_{1}, v_{2}, \cdots, v_{k}\right\} \subseteq$ $V\left(L_{m-1}\right)$ and $w_{1}, w_{2}, \cdots, w_{k} \in V\left(J_{m}\right)$. If there exists a $t$ with $2 \leq t \leq m-1$, such that $V\left(J_{t}\right) \cap\left\{v_{1}, v_{2}, \cdots, v_{k}, u\right\}=\emptyset$, and such that for all $j>t$, either $s_{j}>0$ and $\left(J_{t}, J_{j}\right)_{D}=$ $\emptyset$, or $s_{j}<0$ and $\left(J_{j}, J_{t}\right)_{D}=\emptyset$, then by Lemma 7.3.4(i), $D-V\left(J_{t}\right)=L\left(\mathbf{s}^{\prime}\right)$, where $\mathbf{s}^{\prime}=$ $\left(s_{1}, \cdots, s_{t-1}, s_{t+1}, \cdots, s_{m}\right) \in \mathcal{S}\left(n-s_{t}, k\right)$. By induction, $D-V\left(J_{t}\right) \in \mathcal{D}(k)$, and so $\bar{\lambda}(D+a) \geq$ $\bar{\lambda}\left(\left(D-V\left(J_{t}\right)\right)+a\right) \geq k+1$, contrary to (7.6). Hence we may assume that for any $i$ with $1<t \leq m-1$, there exists a $j>t+1$ such that

$$
0<\left\{\begin{array}{ll}
\left|V\left(J_{t}\right) \cap\left\{v_{1}, v_{2}, \cdots, v_{k}, u\right\}\right|+\left|\left(J_{t}, J_{j}\right)_{D}\right| & \text { if } s_{j}>0 \\
\left|V\left(J_{t}\right) \cap\left\{v_{1}, v_{2}, \cdots, v_{k}, u\right\}\right|+\left|\left(J_{j}, J_{t}\right)_{D}\right| & \text { if } s_{j}<0
\end{array} .\right.
$$

Let $X \subset V(D)$ be a subset satisfying (7.8). Define $I^{\prime}=\left\{i: 1 \leq i \leq m\right.$ and $\left.V\left(J_{i}\right) \cap X=\emptyset\right\}$ and $I^{\prime \prime}=\left\{i: 1 \leq i \leq m\right.$ and $\left.V\left(J_{i}\right) \cap X \neq \emptyset\right\}$.

Claim 1. For any $i$ with $1 \leq i \leq m$, if $\left|V\left(J_{i}\right)\right|=k+2$, then either $X \cap V\left(J_{i}\right)=\emptyset$ or $V\left(J_{i}\right)-X=\emptyset$. (As $\left|s_{i}\right| \in\{1, k+2\}$, it follows that for any $1 \leq i \leq m$, either $X \cap V\left(J_{i}\right)=\emptyset$ or $\left.V\left(J_{i}\right)-X=\emptyset.\right)$

Proof of Claim 1. By contradiction, suppose for some $i^{\prime}$ with $1 \leq i^{\prime} \leq m$ and with $\left|V\left(J_{i^{\prime}}\right)\right|=k+2$, and both $X \cap V\left(J_{i^{\prime}}\right) \neq \emptyset$ and $V\left(J_{i^{\prime}}\right)-X \neq \emptyset$. If $k \geq\left|X \cap V\left(J_{i^{\prime}}\right)\right| \geq 2$, then 
as $J_{i^{\prime}}=H(k, 2)$, we have $\min \left\{\left|X \cap V\left(J_{i^{\prime}}\right)\right|,\left|V\left(J_{i^{\prime}}\right)-X\right|\right\} \geq 2$. It follows by the definition of $H(k, 2)$ that $\left|\partial_{D+a}^{+}(X)\right| \geq\left|\partial_{J_{i^{\prime}}}^{+}\left(X \cap V\left(J_{i^{\prime}}\right)\right)\right| \geq k+1$, contrary to (7.8). Hence we may assume that $\left|X \cap V\left(J_{i^{\prime}}\right)\right| \in\{1, k+1\}$, and so $\left|\partial_{J_{i^{\prime}}}^{+}\left(X \cap V\left(J_{i^{\prime}}\right)\right)\right|=k$. By $(7.7),\left|\{u, v\} \cap V\left(J_{i^{\prime}}\right)\right| \leq 1$ and so $\min \left\{\left|X \cap V\left(J_{i^{\prime}}\right)\right|,\left|V\left(J_{i^{\prime}}\right)-X\right|\right\}=1$. It follows that

$$
\left|\left(X \cap V\left(J_{i^{\prime}}\right), V\left(J_{i^{\prime}}\right)-X\right)_{D+a}\right|=\left|\left(X \cap V\left(J_{i^{\prime}}\right), V\left(J_{i^{\prime}}\right)-X\right)_{D}\right|=\left|\partial_{J_{i^{\prime}}}^{+}\left(X \cap V\left(J_{i^{\prime}}\right)\right)\right|=k .
$$

By (7.10), we must have $\left\{v_{1}, \cdots, v_{k}\right\} \subseteq X \cap V\left(J_{i^{\prime}}\right)$ and $\left\{w_{1}, \cdots, w_{k}\right\} \subseteq V\left(J_{i^{\prime}}\right)-X$. Also by (7.10), for any $j \neq i^{\prime}$, if $X \cap V\left(J_{j}\right) \neq \emptyset$ and $V\left(J_{j}\right)-X \neq \emptyset$, then $\partial_{J_{j}}^{+}\left(X \cap V\left(J_{j}\right)\right) \neq \emptyset$. This, together with (7.10), implies $\left|\partial_{D+a}^{+}(X)\right| \geq\left|\partial_{J_{i^{\prime}}}^{+}\left(X \cap V\left(J_{i^{\prime}}\right)\right)\right|+\left|\partial_{J_{j}}^{+}\left(X \cap V\left(J_{j}\right)\right)\right| \geq k+1$, contrary to (7.8). Hence we have

$$
\text { for any } j \neq i^{\prime} \text {, if } X \cap V\left(J_{j}\right) \neq \emptyset \text {, then } V\left(J_{j}\right) \subseteq X \text {. }
$$

Since $J_{i^{\prime}}=H(k, 2), J_{i^{\prime}}$ has a unique vertex $x_{1}=x^{+}\left(J_{i^{\prime}}\right)$ such that $d_{J_{i^{\prime}}}^{+}\left(x_{1}\right)=k$ and a unique vertex $x_{2}=x^{-}\left(J_{i^{\prime}}\right)$ such that $d_{J_{i^{\prime}}}^{-}\left(x_{2}\right)=k$. It follows by (7.10) that either $V\left(J_{i^{\prime}}\right) \cap X=\left\{x_{1}\right\}$ or $V\left(J_{i^{\prime}}\right)-X=\left\{x_{2}\right\}$.

Assume first that $i^{\prime}>1$ and $i_{1}$ is the smallest integer satisfying $1 \leq i_{1}<i^{\prime}$ such that $i_{1} \in I^{\prime \prime}$. If $i_{1}>1$, then either $s_{i_{1}}>0$, whence by (7.11), $\cup_{1 \leq t \leq i_{1}-1} V\left(J_{t}\right) \cap X=\emptyset$, and so by Definition 7.2.2 or 7.2.3, $\left.\left|\partial_{D+a}^{+}(X)\right| \geq \mid V\left(J_{i_{1}}\right), V\left(J_{1}\right)\right)_{D}|\geq| V\left(J_{1}\right) \mid=k+2$; or $s_{i_{1}}<0$, whence by (7.10) and by Definition 7.2.2 or 7.2.3, $\left|\partial_{D+a}^{+}(X)\right| \geq\left|\left(V\left(J_{i^{\prime}}\right) \cap X, V\left(J_{i^{\prime}}\right)-X\right)_{D}\right|+\left|\left(V\left(J_{i_{1}}\right), L_{i_{1}-1}\right)_{D}\right| \geq k+1$. In either case, a contradiction to (7.8) is obtained. Therefore we assume that $i_{1}=1$. If there exists an $i^{\prime \prime}$ with $1<i^{\prime \prime}<i^{\prime}$ such that $X \cap V\left(J_{i^{\prime \prime}}\right)=\emptyset$, then assume that $i^{\prime \prime}$ is the smallest such integer. By Definition 7.2.2 or 7.2.3, $\left|\left(V\left(L_{i^{\prime \prime}-1}\right), V\left(J_{i^{\prime \prime}}\right)\right)_{D}\right|>0$. This, together with (7.10), implies that $\left|\partial_{D+a}^{+}(X)\right| \geq\left|\left(V\left(J_{i^{\prime}}\right) \cap X, V\left(J_{i^{\prime}}\right)-X\right)_{D}\right|+\left|\left(V\left(L_{i^{\prime \prime}-1}\right), V\left(J_{i^{\prime \prime}}\right)\right)_{D}\right| \geq k+1$, contrary to (7.8). Therefore, no such $i^{\prime \prime}$ exists, and so we conclude that $V\left(L_{i^{\prime}-1}\right) \subseteq X$. It follows by Definition 7.3.1(ii-B) that $\left|\partial_{D+a}^{+}(X)\right| \geq \min \left\{d_{L_{i^{\prime}}}^{+}\left(x_{1}\right), d_{L_{i^{\prime}}}^{-}\left(x_{2}\right)\right\} \geq k+1$, contrary to (7.8).

Therefore, we may assume that $i^{\prime}=1$. If for some $t$ with $1<t \leq m,\left|s_{t}\right|=k+2$, then by Definition 7.3.1(ii-B), we have $\left|\partial_{D+a}^{+}(X)\right| \geq \min \left\{d_{L_{t}}^{+}\left(x_{1}\right), d_{L_{t}}^{-}\left(x_{2}\right)\right\} \geq k+1$, contrary to (7.8). Hence for all $t>1$, we have $\left|s_{t}\right|=1$. It follows by Theorem 7.3.3 that $D \in \mathcal{D}(k)$, contrary to (7.6). This justifies Claim 1.

Claim 2. Suppose that $V\left(J_{1}\right) \cap X=\emptyset$. Let $i_{1}>1$ be the smallest integer such that $V\left(J_{i_{1}}\right) \cap X \neq \emptyset$, and $i_{2} \leq m$ be the largest integer such that for any $t$ with $i_{1} \leq t \leq i_{2}$, we have $V\left(J_{t}\right) \subseteq X$. Each of the following holds.

(i) For any $i \geq 2$, if $V\left(J_{i}\right) \cap X \neq \emptyset$, then $s_{i}<0$.

(ii) $V\left(J_{m}\right) \cap X=\emptyset$.

(iii) $\left(V\left(J_{i_{1}}\right), V\left(L_{i_{1}-1}\right)\right)_{D}=\partial_{D+a}^{+}(X)$ and $\left|\partial_{D+a}^{+}(X)\right|=\left|\left(V\left(J_{i_{1}}\right), V\left(L_{i_{1}-1}\right)\right)_{D}\right|=k$.

(iv) $u \notin X$.

(v) $\bar{\lambda}(D+a) \geq k+1$. (Thus a contradiction to (7.6) is obtained.) 
Proof of Claim 2. (i). Suppose that $V\left(J_{1}\right) \cap X=\emptyset$. By Definition 7.3.1, $\left|V\left(J_{1}\right)\right|=s_{1}=k+2$. If for some for any $i \geq 2$ with $V\left(J_{i}\right) \cap X \neq \emptyset$, we have $s_{i}>0$, then by Definition 7.3.1, for each vertex $x \in V\left(J_{i}\right)$ and for each vertex $y \in V\left(J_{1}\right),(x, y) \in A(D)$. It follows by $\left|V\left(J_{1}\right)\right|=s_{1}=k+2$ and by Claim 1 that $\left|\partial_{D+a}^{+}(X)\right| \geq\left|\left(V\left(J_{i}\right), V\left(J_{1}\right)\right)_{D}\right| \geq k+2$, contrary to (7.8). This justifies (i). (ii). Since $D=\left[L_{m-1}, J_{m}\right]_{k}$, we have $s_{m}>0$ and so by Claim 2(i) and by Claim $1, V\left(J_{m}\right) \cap X=\emptyset$. (iii). By Claim 2(i), $s_{i_{1}}<0$. Thus by Definition 7.3.1(ii), $\left|\left(V\left(J_{i_{1}}\right), V\left(L_{i_{1}-1}\right)\right)_{D}\right|=k$. By the definition of $i_{1}, V\left(L_{i_{1}-1}\right) \cap X=\emptyset$ and $V\left(J_{i_{1}}\right) \subseteq X$. Hence $\left(V\left(J_{i_{1}}\right), V\left(L_{i_{1}-1}\right)\right)_{D} \subseteq \partial_{D+a}^{+}(X)$. By (7.8), we have $\left|\partial_{D+a}^{+}(X)\right|=\left|\left(V\left(J_{i_{1}}\right), V\left(L_{i_{1}-1}\right)\right)_{D}\right|=k$, which implies $\left(V\left(J_{i_{1}}\right), V\left(L_{i_{1}-1}\right)\right)_{D}=$ $\partial_{D+a}^{+}(X)$.

(iv). If $u \in X$, then by Claim 2(ii), we have $(u, v) \in \partial_{D}^{+}(X)$. This, together with Claim 2(iii), implies that $\left|\partial_{D+a}^{+}(X)\right| \geq k+1$, contrary to $(7.8)$.

(v). For any $t>i_{2}$ with $s_{t}>0$, by (7.8) and Claim 2(iii), we must have

$$
\left(X, V\left(J_{t}\right)\right)_{D+a}=\left(\cup_{i \in I^{\prime \prime}} V\left(J_{i}\right), V\left(J_{t}\right)\right)_{D+a}=\emptyset .
$$

Let $\mathbf{s}^{\prime \prime}$ be a subsequence of $\mathbf{s}$ by deleting all terms $s_{i}$ with $i \in I^{\prime \prime}$ from $\mathbf{s}$; and let $D^{\prime \prime}=D-X$. It follows that $D^{\prime \prime}=L\left(\mathbf{s}^{\prime \prime}\right)$ and so $D^{\prime \prime} \in \mathcal{S}(n-|X|, k)$. Since $I^{\prime \prime} \neq \emptyset$, by induction, $D^{\prime \prime} \in \mathcal{E}(k)$. By Claim 2(iv), $u \notin X$ and so both ends $u$ and $v$ are in $V\left(D^{\prime \prime}\right)$. Since $D^{\prime \prime} \in \mathcal{E}(k)$, we have $\bar{\lambda}(D+a) \geq \lambda\left(D^{\prime \prime}+a\right) \geq k+1$. This completes the proof for Claim 2.

Claim 3. Suppose that $V\left(J_{1}\right) \subseteq X$. Let $i_{2} \leq m$ be the largest integer such that for any $t$ with $1 \leq t \leq i_{2}$, we have $V\left(J_{t}\right) \subseteq X$. Each of the following holds.

(i) For any $i>i_{2}$, if $V\left(J_{i}\right) \cap X=\emptyset$, then $s_{i}>0$.

(ii) $\left(V\left(L_{i_{2}}\right), V\left(J_{i_{2}+1}\right)\right)_{D}=\partial_{D+a}^{+}(X)$ and $\left|\partial_{D+a}^{+}(X)\right|=\left|\left(V\left(L_{i_{2}}\right), V\left(J_{i_{2}+1}\right)\right)_{D}\right|=k$.

(iii) $m>i_{2}+1$.

(iv) Suppose that $s_{i_{2}+1}=1$ and $t>i_{2}+1$. Then $V\left(J_{t}\right) \cap X=\emptyset$ if and only if $s_{t}>0$; and $V\left(J_{t}\right) \subseteq X$ if and only if $s_{t}<0$. In particular, $V\left(J_{m}\right) \cap X=\emptyset$ and $u \notin X$.

(v) Let $i_{3}>1$ be the largest integer such that $V\left(J_{i_{3}}\right) \subseteq X$. Then $m-1>i_{3}>i_{2}, V\left(J_{i_{3}}\right) \cap$ $\left\{v_{1}, v_{2}, \cdots, v_{k}, u\right\}=\emptyset$, and for any $h>i_{3},\left(V\left(J_{i_{3}}\right), V\left(J_{h}\right)\right)_{D}=\emptyset$.

Proof of Claim 3. (i). Let $i>i_{2}$ be an index such that $V\left(J_{i}\right) \cap X=\emptyset$. If $s_{i}<0$, then by Definition 7.2 .2 or 7.2 .3 , for any $x \in V\left(L_{i_{2}}\right)$ and for any $y \in V\left(J_{i}\right)$, we have $(x, y) \in A(D)$. It follows that $\left|\partial_{D+a}^{+}(X)\right| \geq\left|\left(V\left(J_{1}\right), V\left(J_{i}\right)\right)_{D}\right| \geq\left|s_{1}\right|=k+2$, contrary to (7.6).

(ii). By Claim 3(i), $s_{i_{2}+1}>0$. By Definition 7.3.1(ii), $\left|\left(V\left(L_{i_{2}}\right), V\left(J_{i_{2}+1}\right)\right)_{D}\right|=k$. By the definition of $i_{2}, V\left(L_{i_{2}}\right) \cap X=\emptyset$ and $V\left(L_{i_{2}}\right) \subseteq X$. Hence $\left(V\left(L_{i_{2}}\right), V\left(J_{i_{2}+1}\right)\right)_{D} \subseteq \partial_{D+a}^{+}(X)$. By (7.8), we have $\left|\partial_{D+a}^{+}(X)\right|=\left|\left(V\left(L_{i_{2}}\right), V\left(J_{i_{2}+1}\right)\right)_{D}\right|=k$, which implies $\left(V\left(L_{i_{2}}\right), V\left(J_{i_{2}+1}\right)\right)_{D}=$ $\partial_{D+a}^{+}(X)$.

(iii). If $i_{2}+1=m$, then we must have $u \in V\left(L_{i_{2}}\right)$, and so $(u, v) \in\left(V\left(L_{i_{2}}\right), V\left(J_{i_{2}+1}\right)\right)_{D+a} \subseteq$ $\partial_{D+a}^{+}(X)$. As $(u, v) \notin\left(V\left(L_{i_{2}}\right), V\left(J_{i_{2}+1}\right)\right)_{D}$, this yields a contradiction to $\left(V\left(L_{i_{2}}\right), V\left(J_{i_{2}+1}\right)\right)_{D}=$ $\partial_{D+a}^{+}(X)$. 
(iv). Suppose that $s_{i_{2}+1}=1$ and fix $t>i_{2}+1$. Assume that $V\left(J_{t}\right) \cap X=\emptyset$. By Claim 3(ii) and by (7.6), $\left(V\left(J_{t}\right), X\right)_{D}=\emptyset$. Hence by the definition of $\left[L_{t-1}, J_{t}\right]_{k}$, we must have $s_{t}>0$. Conversely, assume that both $s_{t}>0$ and $V\left(J_{t}\right) \subseteq X$, then by the definition of $\left[L_{t-1}, J_{t}\right]_{k}$, $\left(V\left(J_{k}\right), V\left(L_{t-1}\right)\right)_{D} \neq \emptyset$, contrary to Claim 3(ii). This proves that $V\left(J_{t}\right) \cap X=\emptyset$ if and only if $s_{t}>0$.

Now assume that $V\left(J_{t}\right) \subseteq X$. If $s_{t}>0$, then $\left(V\left(J_{t}\right), V\left(J_{i_{2}+1}\right)\right)_{D} \neq \emptyset$, by the dentition of $\left[L_{t-1}, J_{t}\right]_{k}$, contrary to Claim 3(ii). Therefore, we must have $s_{t}<0$. Conversely, assume that $s_{t}<0$ and $V\left(J_{t}\right) \cap X=\emptyset$. By the dentition of $\left[J_{t}, L_{t-1}\right]_{k}$, we have $\left(V\left(L_{t-1}\right), V\left(J_{t}\right)\right)_{D} \neq \emptyset$, again contrary to Claim 3(ii).

As $D=\left[L_{m-1}, J_{m}\right]_{k}$, we have $s_{m}>0$, and so $V\left(J_{m}\right) \cap X=\emptyset$. By Claim 3(ii) and since $v \in V\left(J_{m}\right)$, we conclude that $u \notin X$. This proves (iv).

(v). By Claim 3(iv), $V\left(J_{m}\right) \cap X=\emptyset$, and so $m>i_{3}$. We argue by contradiction to assume that $i_{3}=i_{2}$. Then by the definitions of $i_{2}$ and $i_{3}$, we have $X=\cup_{t=1}^{i_{3}} V\left(J_{t}\right)=V\left(L_{i_{3}}\right)$. For any $j>i_{3}$, by Claim 3(i), $s_{j}>0$. By Claim 3(iv), $u \in X$. If $m=i_{3}+1$, then $u$ must be in $X$, a contradiction. Hence $m \geq i_{3}+2$. Similarly, by $k \geq \mid \partial_{D+a}^{+}(X)$, we also conclude that $\left\{v_{1}, v_{2}, \ldots, v_{k}\right\} \mathcal{X}=\emptyset$. By Claim 3(i), $s_{i_{3}+2}>0$. Since $\left(L_{i_{3}}, J_{i_{3}+1}\right)_{D} \cup\left(L_{i_{3}}, J_{i_{3}+2}\right)_{D} \subseteq \partial_{D+a}^{+}(X)$ and since $\left|\left(L_{i_{3}}, J_{i_{3}+1}\right)_{D}\right|=k$, it follows by $k \geq\left|\partial_{D+a}^{+}(X)\right|$ that $\left|\left(L_{i_{3}}, J_{i_{3}+2}\right)_{D}\right|=0$. This, together with $\left\{v_{1}, v_{2}, \ldots, v_{k}, u\right\} \mathcal{X}=\emptyset$, yields a contradiction to (7.9). This proves that $m>i_{3}>i_{2}$.

We now show the other conclusions of Claim 3(v). By Claim 3(iv), $V\left(J_{m}\right) \cap X=\emptyset$ and $u \notin X$. By Definition 7.3.1 that, we have $\left(J_{i_{3}}, J_{i_{3}+1}\right)_{D} \subseteq\left(J_{i_{3}}, J_{i_{3}+1} \cup J_{m}\right)_{D} \subseteq \partial_{D+a}^{+}(X)$, which implies that

$$
k=\left|\left(J_{i_{3}}, J_{i_{3}+1}\right)_{D}\right| \leq\left|\left(J_{i_{3}}, J_{i_{3}+1}\right)_{D}\right|+\left|\left(J_{i_{3}} \cup J_{m}\right)_{D}\right| \leq\left|\partial_{D+a}^{+}(X)\right| \leq k .
$$

Thus $\left|\left(J_{i_{3}} \cup J_{m}\right)_{D}\right|=0$. Since $w_{1}, w_{2}, \cdots, w_{k} \in V\left(J_{m}\right)$, it follows that $V\left(J_{i_{3}}\right) \cap\left\{v_{1}, v_{2}, \cdots, v_{k}, u\right\}$ $=\emptyset$. By the choice of $i_{3}$, for any $h>i_{3}$, we have $V\left(J_{h}\right) \cap X=\emptyset$, and so $\left(V\left(J_{i_{3}}\right), V\left(J_{h}\right)\right)_{D} \subseteq$ $\partial_{D+a}^{+}(X)$. By Claim 3(ii), we must have $\left(V\left(J_{i_{3}}\right), V\left(J_{h}\right)\right)_{D}=\emptyset$. This justifies Claim 3.

We now continue the proof of the theorem. By Claim $2(\mathrm{v})$, we may assume that $s_{1}=-(k+2)$, and so Claim 3 applies. By Claim 3(iv) and with $i_{3}$ being defined in Claim 3(v), we conclude that $s_{h}>0$, for any $h>i_{3}$. Therefore, Claim 3(v) presents a contradiction to (7.9). This proves the theorem.

To determine the extremal graphs of Theorem 7.1.4, we need to construct a new family of digraphs.

Definition 7.3.6. For given integer $k>0$, define $\mathcal{E}_{1}(k)$ to be the family consisting of digraphs satisfying each of the following.

(A) $\mathcal{E}(k) \subset \mathcal{E}_{1}(k)$.

(B) If digraphs $H$ and $H^{\prime}$ satisfy

$$
H, H^{\prime} \in \mathcal{E}_{1}(k) \cup\left\{K_{1}\right\} \text { with }|V(H)|+\left|V\left(H^{\prime}\right)\right|>2,
$$


then $\left[H, H^{\prime}\right]_{k} \subset \mathcal{E}_{1}(k)$.

Lemma 7.3.7. For any $D \in \mathcal{E}_{1}(k)$.

(i) $|V(D)| \geq k+2$.

(ii) $\lambda(D)=k$.

(iii) For any $k$-arc-cut $W$ of $D$, there exist two digraphs $H$ and $H^{\prime}$ satisfying (7.12) such that $D \in\left[H, H^{\prime}\right]_{k}$ and $W=\left(V(H), V\left(H^{\prime}\right)\right)_{D}$.

Proof. By Definition 7.3.6 and by induction on $|V(D)|$ for a digraph $D \in \mathcal{E}_{1}(k)$, Lemma 7.3.7(i) and (ii) hold. To prove Lemma 7.3.7(iii), we assume that $D$ has a $k$-arc-cut $W=$ $\left\{\left(u_{1}, v_{1}\right),\left(u_{2}, v_{2}\right), \ldots,\left(u_{k}, v_{k}\right)\right\}$. Thus for some nonempty subsets $X, V(D)-X$, we have $W=$ $(X, V(D)-X)_{D}$. If $D \in \mathcal{E}(k)$, then by Observation 2(ii), Lemma 7.3.7 (iii) must hold. Hence by Definition 7.3.6, we assume that $D \in\left[H, H^{\prime}\right]_{k}$ for some $H, H^{\prime}$ satisfying (7.12); and that Lemma 7.3.7(iii) holds for digraphs in $\mathcal{E}_{1}(k)$ with smaller order than $D$. Let $Z=\left(V(H), V\left(H^{\prime}\right)\right)_{D}$.

Case 1. $X \cap V\left(H^{\prime}\right)=\emptyset$, or $(V(D)-X) \cap V\left(H^{\prime}\right)=\emptyset$.

By symmetry, we assume that $X \cap V\left(H^{\prime}\right)=\emptyset$. Then $X$ is a $k$-arc-cut of $H$. By induction, there exist digraphs $L, L^{\prime}$ satisfying (7.12) such that $H \in\left[L, L^{\prime}\right]_{k}$ and $W=\left(V(L), V\left(L^{\prime}\right)\right)_{H}$. As $X \cap V\left(H^{\prime}\right)=\emptyset$, we have $V(L)=X$. Since $W$ is an arc-cut of $D, W \mathcal{Z}=\emptyset$ and so $D \in\left[L, L^{\prime \prime}\right]_{k}$ with $W=\left(V(L), V\left(L^{\prime \prime}\right)\right)_{D}, L^{\prime \prime}=D-X \in\left[L^{\prime}, H^{\prime}\right]_{k}$ and $Z=(V(L), V(D)-X)_{L^{\prime \prime}}$. Since $L^{\prime}, H^{\prime} \in \mathcal{E}_{1}(k) \cup\left\{K_{1}\right\}$, it follows by Definition 7.3.6 that $L^{\prime \prime} \in \mathcal{E}_{1}(k)$. This implies that Lemma 7.3.7(iii) holds.

Case 2. $X \cap V\left(H^{\prime}\right) \neq \emptyset$ and $(V(D)-X) \cap V\left(H^{\prime}\right) \neq \emptyset$.

Let $W_{1}=(X \cap V(H), V(H)-X)_{H}$ and $W_{2}=\left(X \cap V\left(H^{\prime}\right), V\left(H^{\prime}\right)-X\right)_{H^{\prime}}$. Thus $W=W_{1} \cup W_{2}$ and $\left|W_{1}\right|+\left|W_{2}\right|=|W|=k$. If both $H, H^{\prime} \in \mathcal{E}_{1}(k)$, then by Lemma 7.3.7(ii), we must have $\left|W_{1}\right| \geq k$ and $\left|W_{2}\right| \geq k$, contrary to the fact that $\left|W_{1}\right|+\left|W_{2}\right|=|W|=k$. Hence either $H=K_{1}$ or $H^{\prime}=K_{1}$. Suppose that $H=K_{1}$ with $V(H)=\{v\}$. By the definition of $\left[H, H^{\prime}\right]_{k}$, for any $v^{\prime} \in X \cap V\left(H^{\prime}\right),\left(v^{\prime}, v\right) \in A(D)$.

Thus if $v \notin X$, then $X \subset V\left(H^{\prime}\right)$ and so $W \subseteq(X,\{v\})_{D} \cup\left(X, V\left(H^{\prime}\right)-X\right)_{D}$. It follows from Lemma 7.3.7(ii) that $k=|W|=\left|(X,\{v\})_{D}\right|+\left|\left(X, V\left(H^{\prime}\right)-X\right)_{D}\right| \geq\left|(X,\{v\})_{D}\right|+k$, and so $(X,\{v\})_{D}=\emptyset$ and $D \in\left[\{v\}, H^{\prime}\right]_{k}$. By induction, there exist digraphs $L, L^{\prime}$ satisfying (7.12) such that $H^{\prime} \in\left[L, L^{\prime}\right]_{k}$ and $W=\left(V(L), V\left(L^{\prime}\right)\right)_{H}$. Let $L^{\prime \prime} \in\left[\{v\}, L^{\prime}\right]_{k}$. Then $L^{\prime \prime} \in \mathcal{E}_{1}(k)$ and $D \in\left[L, L^{\prime \prime}\right]_{k}$ with $W=\left(V(L), V\left(L^{\prime \prime}\right)\right)_{D}$. Hence Lemma 7.3.7(iii) holds.

Therefore, we must have $v \in X$, which implies that $\left(\{v\}, V\left(H^{\prime}\right)-X\right)_{D} \neq \emptyset$. It follows that $k=|W|=\left|\left(\{v\}, V\left(H^{\prime}\right)-X\right)_{D}\right|+\left|\left(X-\{v\}, V\left(H^{\prime}\right)-X\right)_{D}\right|>\left|\left(X-\{v\}, V\left(H^{\prime}\right)-X\right)_{D}\right|$. This implies that $\lambda\left(H^{\prime}\right) \leq\left|\left(X-\{v\}, V\left(H^{\prime}\right)-X\right)_{D}\right|<k$, contrary to Lemma 7.3.7(ii). This completes the proof of the lemma.

Lemma 7.3.8. For any integer $k>1$, we have $\mathcal{E}_{1}(k) \subseteq \mathcal{D}(k)$. 
Proof. Let $D \in \mathcal{E}_{1}(k)$. We need to show that $D \in \mathcal{D}(k)$. If $D \in \mathcal{E}(k)$, then by Theorem 7.3.5, $D \in \mathcal{E}(k)$. Hence we assume that $D \in \mathcal{E}_{1}(k)-\mathcal{E}(k)$, and Lemma 7.3.8 holds for graphs in $\mathcal{E}_{1}(k)$ with smaller order.

For any $e \in A\left(D^{c}\right)$, if $\lambda(D+e) \geq k+1$, then $D \in \mathcal{D}(k)$. Hence we assume that $\lambda(D+e) \leq k+1$. Let $W$ be a $j$-arc-cut of $D+e$ for some $j \leq k$. By Lemma 7.3.7(ii), $e \notin W$ and so by Lemma 7.3.7(iii), for some digraphs $H, H^{\prime}$ satisfying (7.12), $D \in[H, H]_{k}$ and $W=\left(V(H), V\left(H^{\prime}\right)\right)_{D}$. Let $e=(u, v)$. Since $e \notin W$, we cannot have $u \in V(H)$ and $v \in V\left(H^{\prime}\right)$. By the definition of $\left[H, H^{\prime}\right]_{k}$, we cannot have $v \in V(H)$ and $u \in V\left(H^{\prime}\right)$. Hence either $u, v \in V(H)$ or $u, v \in V\left(H^{\prime}\right)$. Without loss of generality, we assume that $u, v \in V(H)$, and so $e \in A\left(H^{c}\right)$. By (7.12), $H \in \mathcal{E}_{1}(l)$ and so by induction, $\bar{\lambda}(H+e) \geq k+1$. It follows that $\bar{\lambda}(D+e) \geq \bar{\lambda}(H+e) \geq k+1$, and so by definition, $D \in \mathcal{D}(k)$.

Definition 7.3.9. Let $n$ and $k$ be integers with $n>k>0$ and $q, r$ be nonnegative integers satisfying $n=q(k+2)+r$ with $0 \leq r \leq k+1$,

(i) Define $\mathcal{S}^{\prime}(n, k)$ to be the set of all integral sequences $\left(s_{1}, s_{2}, \cdots, s_{q+r}\right)$ such that $s_{1}=k+2$, and for $i \geq 2,\left|s_{i}\right| \in\{1, k+2\}$. Note that if $\left(s_{1}, s_{2}, \cdots, s_{q+r}\right) \in \mathcal{S}^{\prime}(n, k)$, then as $q(k+2)+r=$ $n=\sum_{i=1}^{q+r}\left|s_{i}\right|$, there are exactly $r$ of the $\left|s_{i}\right|$ 's equaling one and $q$ of the $\left|s_{i}\right|$ 's equaling $k+2$. Define $\mathcal{E}^{\prime}(n, k)=\left\{L(\mathbf{s}): \mathbf{s} \in \mathcal{S}^{\prime}(n, k)\right\}$ and $\mathcal{E}^{\prime}(k)=\cup_{n \geq k+2} \mathcal{E}^{\prime}(n, k)$.

(ii) Define $\mathcal{E}_{1}^{\prime}(k)$ to be the family consisting of digraphs satisfying each of the following. (ii-A) $\mathcal{E}^{\prime}(k) \subset \mathcal{E}_{1}^{\prime}(k)$.

(ii-B) For $H, H^{\prime} \in \mathcal{E}_{1}^{\prime}(k) \cup\left\{K_{1}\right\}$ satisfying $\left|V\left(H_{1}\right)\right|+\left|V\left(H_{2}\right)\right|>2$ and $\left\lfloor\frac{n}{k+2}\right\rfloor=\left\lfloor\frac{\left|V\left(H_{1}\right)\right|}{k+2}\right\rfloor+$ $\left\lfloor\frac{\left|V\left(H_{2}\right)\right|}{k+2}\right\rfloor,\left[H, H^{\prime}\right]_{k} \subset \mathcal{E}_{1}^{\prime}(k)$.

By Definition 7.3.9, the corollary below follows immediately from Theorem 7.3.5 and Lemma 7.3.8.

Corollary 7.3.10. $\mathcal{E}_{1}^{\prime}(k) \subseteq \mathcal{D}(k)$

Given the structure of digraphs in $\mathcal{E}_{1}^{\prime}(k)$, we can compute the size of digraphs in $\mathcal{E}_{1}^{\prime}(k)$.

Lemma 7.3.11. Let $n>k+1 \geq 2$ be integers. For any digraph $D \in \mathcal{E}_{1}^{\prime}(k)$, we have

$$
|A(D)|=\left(\begin{array}{l}
n \\
2
\end{array}\right)+(n-1) k+\left\lfloor\frac{n}{k+2}\right\rfloor\left(1+2 k-\left(\begin{array}{c}
k+2 \\
2
\end{array}\right)\right) .
$$

Proof. We first assume that $D \in \mathcal{E}^{\prime}(k)$ with $|V(D)|=n$ and $n>k+1 \geq 2$. If $n=k+2$, then by Definition 7.3.9, we have $D=H(k, 2)$, and so $|A(D)|=(k+2)(k+1)-1$. Thus (7.13) holds. Assume that $n>k+2$ and (7.13) holds for smaller values of $n$. Let $q, r$ be nonnegative integers satisfying $n=q(k+2)+r$ with $0 \leq r \leq k+1$. By Definitions 7.3.1 and 7.3.9, we have $\left|s_{q+r}\right| \in\{1, k+2\}$. 
Case 1. $\left|s_{q+r}\right|=1$.

By Definition 7.3.9, we may assume that $s_{q+r}=1$ and $D \in\left[H, K_{1}\right]_{k}$ for some $H \in \mathcal{E}^{\prime}(k)$. Denote $V\left(K_{1}\right)=\{v\}$. Since $s_{q+r}=1$, we have $r \geq 1$, and so $n-1=q(k+2)+r-1$, which implies $\left\lfloor\frac{n}{k+2}\right\rfloor=\left\lfloor\frac{n-1}{k+2}\right\rfloor$. By induction, we have

$$
\begin{aligned}
|A(D)| & =|A(H)|+k+(n-1) \\
& =\left(\begin{array}{c}
n-1 \\
2
\end{array}\right)+(n-2) k+\left\lfloor\frac{n-1}{k+2}\right\rfloor\left(1+2 k-\left(\begin{array}{c}
k+2 \\
2
\end{array}\right)\right)+k+(n-1) \\
& =\left(\begin{array}{c}
n \\
2
\end{array}\right)+(n-1) k+\left\lfloor\frac{n}{k+2}\right\rfloor\left(1+2 k-\left(\begin{array}{c}
k+2 \\
2
\end{array}\right)\right) .
\end{aligned}
$$

Case 2. $\left|s_{q+r}\right|=k+2$.

By Definition 7.3.9, we may assume that $s_{q+r}=k+2$ and $D=[H, H(k, 2)]_{k}$ for some $H \in \mathcal{E}^{\prime}(k)$. Since $s_{1}=k+2$ and $s_{q+r}=k+2$, we have $q \geq 2$, and so $n-(k+2)=(q-1)(k+2)+r$, which implies $\left\lfloor\frac{n}{k+2}\right\rfloor=\left\lfloor\frac{n-(k+2)}{k+2}\right\rfloor+1$. By induction, we have

$$
\begin{aligned}
|A(D)|= & |A(H)|+k+(n-(k+2))(k+2)+|A(H(k, 2))| \\
= & \left(\begin{array}{c}
n-(k+2) \\
2
\end{array}\right)+(n-(k+2)-1) k+\left\lfloor\frac{n-(k+2)}{k+2}\right\rfloor\left(1+2 k-\left(\begin{array}{c}
k+2 \\
2
\end{array}\right)\right) \\
& +k+[n-(k+2)](k+2)+(k+2)(k+1)-1 \\
= & \left(\begin{array}{l}
n \\
2
\end{array}\right)+(n-1) k+\left\lfloor\frac{n}{k+2}\right\rfloor\left(1+2 k-\left(\begin{array}{c}
k+2 \\
2
\end{array}\right)\right) .
\end{aligned}
$$

Thus, (7.13) holds for any $D \in \mathcal{E}^{\prime}(k)$. Next, we assume that $D \in \mathcal{E}_{1}^{\prime}(k)-\mathcal{E}^{\prime}(k)$. By Definition 7.3.9, there exists $H, H^{\prime} \in \mathcal{E}_{1}^{\prime}(k)$ satisfying Definition 7.3.9 (ii-B). Let $n_{1}=|V(H)|$ and $n_{2}=\left|V\left(H^{\prime}\right)\right|$. Thus $n=n_{1}+n_{2}$ and $\left\lfloor\frac{n}{k+2}\right\rfloor=\left\lfloor\frac{n_{1}}{k+2}\right\rfloor+\left\lfloor\frac{n_{2}}{k+2}\right\rfloor$. By induction, we have

$$
\begin{aligned}
|A(D)|= & |A(H)|+k+n_{1} n_{2}+\left|A\left(H^{\prime}\right)\right| \\
& \left(\begin{array}{c}
n_{1} \\
2
\end{array}\right)+\left(n_{1}-1\right) k+\left\lfloor\frac{n_{1}}{k+2}\right\rfloor\left(1+2 k-\left(\begin{array}{c}
k+2 \\
2
\end{array}\right)\right)+k+n_{1} n_{2} \\
& +\left(\begin{array}{c}
n_{2} \\
2
\end{array}\right)+\left(n_{2}-1\right) k+\left\lfloor\frac{n_{2}}{k+2}\right\rfloor\left(1+2 k-\left(\begin{array}{c}
k+2 \\
2
\end{array}\right)\right) \\
= & \left(\begin{array}{c}
n_{1} \\
2
\end{array}\right)+\left(\begin{array}{c}
n_{2} \\
2
\end{array}\right)+n_{1} n_{2}+(n-1) k+\left\lfloor\frac{n}{k+2}\right\rfloor\left(1+2 k-\left(\begin{array}{c}
k+2 \\
2
\end{array}\right)\right) \\
= & \left(\begin{array}{c}
n \\
2
\end{array}\right)+(n-1) k+\left\lfloor\frac{n}{k+2}\right\rfloor\left(1+2 k-\left(\begin{array}{c}
k+2 \\
2
\end{array}\right)\right) .
\end{aligned}
$$

By induction, (7.13) holds for any $D \in \mathcal{E}_{1}^{\prime}(k)$.

The following lemma gives us more information on the structure of digraphs in $\mathcal{D}(k)$.

Lemma 7.3.12. Let $k \geq 2$ be an integer. If $D \in \mathcal{D}(k)$ and if for some $H_{1}, H_{2} \in \mathcal{D}(k)$, we have $D \in\left[H_{1}, H_{2}\right]_{k}$, then for each $i \in\{1,2\}, H_{i} \neq K_{k+1}^{*}$. 
proof. By contradiction, we assume that $H_{2} \cong K_{k+1}^{*}$ and $D \in\left[H_{1}, H_{2}\right]_{k}$ and that $D \in \mathcal{D}(k)$. Let $V\left(H_{2}\right)=\left\{v_{1}, v_{2}, \cdots, v_{k+1}\right\}$. By Definition 7.2 .2 , we may assume that $\left|\left(H_{1}, H_{2}\right)_{D}\right|=k$, and so we may assume that $N_{D}^{+}\left(V\left(H_{1}\right), V\left(H_{2}\right)\right) \subseteq\left\{v_{1}, v_{2}, \cdots, v_{k}\right\}$. Since $H_{1}, H_{2} \in \mathcal{D}(k)$, both $\left|V\left(H_{1}\right)\right| \geq k+1$ and $\left|V\left(H_{2}\right)\right| \geq k+1$. Thus there must be a vertex $u \in V\left(H_{1}\right)$ and an integer $i$ with $1 \leq i \leq k$, such that $a=\left(u, v_{i}\right) \notin A(D)$. Since $D \in \mathcal{D}(k), D+a$ has a subdigraph $D^{\prime}$ with $\lambda\left(D^{\prime}\right) \geq k+1$. Note that $d_{D+a}^{-}\left(v_{k+1}\right)=d_{D}^{-}\left(v_{k+1}\right)=k, v_{k+1} \notin V\left(D^{\prime}\right)$. Since, for each $j$ with $1 \leq j \leq k$ and $j \neq i, d_{D+a-v_{k+1}}^{-}\left(v_{j}\right) \leq d_{D-v_{k+1}}^{-}\left(v_{j}\right)+1 \leq k$, it follows that $v_{j} \notin V\left(D^{\prime}\right)$ for each $j$ with $1 \leq j \leq k$ and $j \neq i$. Since $k \geq 2, d_{D+a-v_{k+1}}^{-}\left(v_{i}\right) \leq k$, and $v_{i} \notin V\left(D^{\prime}\right)$ as well. This implies that $a \notin A\left(D^{\prime}\right)$, and so $D^{\prime} \subseteq D$. Contrary to the assumption that $D \in \mathcal{D}(k)$. This proves the lemma.

\subsection{The Extremal Function}

The main result of this section is Theorem 7.4.1, which clearly implies Theorem 7.1.4.

Theorem 7.4.1. Let $n, k$ be integers with $n>k+1 \geq 2$. Then for any $D \in \mathcal{D}(n, k)$, we have

$$
|A(D)| \geq\left(\begin{array}{l}
n \\
2
\end{array}\right)+(n-1) k+\left\lfloor\frac{n}{k+2}\right\rfloor\left(1+2 k-\left(\begin{array}{c}
k+2 \\
2
\end{array}\right)\right) .
$$

Furthermore, equality holds in (7.14) if and only if $D \in \mathcal{E}_{1}^{\prime}(k)$.

Proof. We argue by induction to prove (7.14) on $n=|V(D)|$. If $n=k+2$, then $D=H(k, 2)$. Thus we have $|A(D)|=(k+2)(k+1)-1$, and so (7.14) holds. Assume that $n>k+2$ and (7.14) holds for smaller values of $n$. Let $q, r \geq 0$ be integers satisfying $n=q(k+2)+r$ with $0 \leq r \leq k+1$.

As $n>k+2, D \neq K_{k+2}^{*}$. By Lemma 7.2.4, one of the three conclusions of Lemma 7.2.4 must hold.

Claim 1. If Lemma 7.2.4(i) or (ii) holds, then (7.14) holds as well. Moreover, if $r=0$, then (7.14) holds with strict inequality.

Without lose of generality, we assume that $D \in\left[H, K_{1}\right]_{k}$ for some $H \in \mathcal{D}(k)$ with $V\left(K_{1}\right)=$ $\{v\}$. As $|V(D)|=n-1$, by Definition $7.2 .2,\left|\partial_{D}^{+}(v)\right|=k$ and $\left|\partial_{D}^{-}(v)\right|=n-1$.

Case 1: $r=0$. 
Then $q-1=\left\lfloor\frac{n-1}{k+2}\right\rfloor$. By induction, we have

$$
\begin{aligned}
|A(D)| & =|A(H)|+k+(n-1) \\
& \geq\left(\begin{array}{c}
n-1 \\
2
\end{array}\right)+(n-2) k+(q-1)\left(1+2 k-\left(\begin{array}{c}
k+2 \\
2
\end{array}\right)\right)+k+(n-1) \\
& =\left(\begin{array}{l}
n \\
2
\end{array}\right)+(n-1) k+(q-1)\left(1+2 k-\left(\begin{array}{c}
k+2 \\
2
\end{array}\right)\right) \\
& >\left(\begin{array}{l}
n \\
2
\end{array}\right)+(n-1) k+q\left(1+2 k-\left(\begin{array}{c}
k+2 \\
2
\end{array}\right)\right) .
\end{aligned}
$$

Thus (7.14) holds with strict inequality in this case.

Case 2: $r>0$.

Then $q=\left\lfloor\frac{n-1}{k+1}\right\rfloor$. By induction,

$$
\begin{aligned}
|A(D)| & =|A(H)|+k+(n-1) \\
& \geq\left(\begin{array}{c}
n-1 \\
2
\end{array}\right)+(n-2) k+q\left(1+2 k-\left(\begin{array}{c}
k+2 \\
2
\end{array}\right)\right)+k+(n-1) \\
& =\left(\begin{array}{c}
n \\
2
\end{array}\right)+(n-1) k+q\left(1+2 k-\left(\begin{array}{c}
k+2 \\
2
\end{array}\right)\right) .
\end{aligned}
$$

Thus (7.14) holds in this case as well, and so Claim 1 follows.

By Claim 1, we may assume that Lemma 7.2.4(iii) holds. Thus $D \in\left\{\left[H_{1}, H_{2}\right]_{k},\left[H_{2}, H_{1}\right]_{k}\right\}$ for some $H_{1}, H_{2} \in \mathcal{D}(k)$. Let $n_{1}=\left|V\left(H_{1}\right)\right|$ and $n_{2}=\left|V\left(H_{2}\right)\right|$. Then $n=n_{1}+n_{2}$. Without lose of generality, we assume that $n_{1} \geq n_{2}$. By Lemma 7.3.12, $n_{2} \geq k+2$. Let $q_{1}, q_{2} \geq 1, r_{1}, r_{2}$ be integers satisfying $n_{1}=q_{1}(k+2)+r_{1}, 0 \leq r_{1} \leq k+1$, and $n_{2}=q_{2}(k+2)+r_{2}, 0 \leq r_{2} \leq k+1$. Thus $q_{1}=\left\lfloor\frac{n_{1}}{k+2}\right\rfloor$ and $q_{2}=\left\lfloor\frac{n_{2}}{k+2}\right\rfloor$.

Claim 2. If Lemma 7.2.4(iii) holds, then (7.14) holds. Moreover, if $r_{1}+r_{2} \geq k+2$, then (7.14) holds with strict inequality.

Since $n=n_{1}+n_{2}=\left(q_{1}+q_{2}\right)(k+2)+\left(r_{1}+r_{2}\right)$, we observe that $r_{1}+r_{2} \leq k+1$ if and only if $q_{1}+q_{2}=q$, and if and only if $r=r_{1}+r_{2}$. With this observation, we consider the following two cases. Note that if $n_{1} \geq 2$ and $n_{2} \geq 2$, then $\left(\begin{array}{c}n_{1} \\ 2\end{array}\right)+\left(\begin{array}{c}n_{2} \\ 2\end{array}\right)+n_{1} n_{2}=\left(\begin{array}{c}n \\ 2\end{array}\right)$.

Case 1: $r_{1}+r_{2} \leq k+1$.

Then $q_{1}+q_{2}=q$. By Induction,

$$
\begin{aligned}
|A(D)|= & \left|A\left(H_{1}\right)\right|+k+n_{1} n_{2}+\left|A\left(H_{2}\right)\right| \\
\geq & \left(\begin{array}{c}
n_{1} \\
2
\end{array}\right)+\left(n_{1}-1\right) k+q_{1}\left(1+2 k-\left(\begin{array}{c}
k+2 \\
2
\end{array}\right)\right)+k+n_{1} n_{2}+\left(\begin{array}{c}
n_{2} \\
2
\end{array}\right) \\
& +\left(n_{2}-1\right) k+q_{2}\left(1+2 k-\left(\begin{array}{c}
k+2 \\
2
\end{array}\right)\right) \\
= & \left(\begin{array}{c}
n \\
2
\end{array}\right)+(n-1) k+q\left(1+2 k-\left(\begin{array}{c}
k+2 \\
2
\end{array}\right)\right) .
\end{aligned}
$$


Hence (7.14) holds in this case.

Case 2: $r_{1}+r_{2} \geq k+2$.

Then $q_{1}+q_{2}=q-1$ and $r=r_{1}+r_{2}-(k+2)$. Observe that for any $k \geq 1,1+2 k<\left(\begin{array}{c}k+2 \\ 2\end{array}\right)$, and so by induction,

$$
\begin{aligned}
|A(D)|= & \left|A\left(H_{1}\right)\right|+k+n_{1} n_{2}+\left|A\left(H_{2}\right)\right| \\
\geq & \left(\begin{array}{c}
n_{1} \\
2
\end{array}\right)+\left(n_{1}-1\right) k+q_{1}\left(1+2 k-\left(\begin{array}{c}
k+2 \\
2
\end{array}\right)\right)+k+n_{1} n_{2}+\left(\begin{array}{c}
n_{2} \\
2
\end{array}\right) \\
& +\left(n_{2}-1\right) k+q_{2}\left(1+2 k-\left(\begin{array}{c}
k+2 \\
2
\end{array}\right)\right) \\
= & \left(\begin{array}{c}
n \\
2
\end{array}\right)+(n-1) k+(q-1)\left(1+2 k-\left(\begin{array}{c}
k+2 \\
2
\end{array}\right)\right) \\
> & \left(\begin{array}{c}
n \\
2
\end{array}\right)+(n-1) k+q\left(1+2 k-\left(\begin{array}{c}
k+2 \\
2
\end{array}\right)\right) .
\end{aligned}
$$

Thus (7.14) holds with strict inequality in this case, and so Claim 2 is justified.

Claim 3. If equality holds in (7.14) for a digraph $D \in \mathcal{D}(k, n)$, then $D \in \mathcal{E}_{1}^{\prime}(k)$.

Let $D \in \mathcal{D}(k, n)$ be a digraph satisfying equality in (7.14). We argue by induction on $n=|V(D)| \geq k+2$. If $n=k+2$, then $D=H(k, 2) \in \mathcal{E}^{\prime}(k)$. Assume that $n>k+2$ and that Claim 3 holds for smaller values of $n$. Since $n>k+2$, by Lemma 7.2.4, one of the conclusions of Lemma 7.2.4 must hold.

If $D$ satisfies Lemma 7.2.4(i) or (ii), without loss of generality, we assume that $D \in\left[H, K_{1}\right]_{k}$ for some $H \in \mathcal{E}^{\prime}(k)$ with $V\left(K_{1}\right)=v$. By Claim 1, if equality holds in (7.14), then $r>0$, which implies that $n-1=q(k+2)+(r-1)$, with $0 \leq r-1 \leq k$. Since equality in (7.14) holds, it follows by (7.15) that $|A(H)|=\left(\begin{array}{c}n-1 \\ 2\end{array}\right)+(n-2) k+(q-1)\left(1+2 k-\left(\begin{array}{c}k+2 \\ 2\end{array}\right)\right)$. By induction, $H \in \mathcal{E}_{1}^{\prime}(n-1, k)$. By Definition 7.3.9, $D \in \mathcal{E}^{\prime}(n, k)$, and so $D \in \mathcal{E}^{\prime}(k)$ in this case.

Hence we may assume that $D$ satisfies Lemma 7.2.4(iii), and so $D \in\left[H_{1}, H_{2}\right]_{k}$ for some $H_{1}, H_{2} \in \mathcal{E}^{\prime}(k)$. Again, let $n_{1}=\left|V\left(H_{1}\right)\right|$ and $n_{2}=\left|V\left(H_{2}\right)\right|$; and let $q_{1}, q_{2} \geq 1, r_{1}, r_{2}$ be integers satisfying $n_{1}=q_{1}(k+2)+r_{1}, 0 \leq r_{1} \leq k+1$, and $n_{2}=q_{2}(k+2)+r_{2}, 0 \leq r_{2} \leq k+1$. By Claim 2 , if equality holds in (7.14), then $r_{1}+r_{2} \leq k+1$, which implies that $q=q_{1}+q_{2}$ and $r=r_{1}+r_{2}$. Since equality in (7.14) holds, it follows by (7.15) that both $\left|A\left(H_{1}\right)\right|=\left(\begin{array}{c}n_{1} \\ 2\end{array}\right)+\left(n_{1}-1\right) k+$ $q_{1}\left(1+2 k-\left(\begin{array}{c}k+2 \\ 2\end{array}\right)\right)$ and $\left|A\left(H_{2}\right)\right|=\left(\begin{array}{c}n_{2} \\ 2\end{array}\right)+\left(n_{2}-1\right) k+q_{2}\left(1+2 k-\left(\begin{array}{c}k+2 \\ 2\end{array}\right)\right)$. Therefore by induction, $H_{1}, H_{2} \in \mathcal{E}_{1}^{\prime}(k)$. By Definition $7.3 .9, D \in\left[H_{1}, H_{2}\right]_{k}$, which is in $\mathcal{E}_{1}^{\prime}(n, k)$, and so $D \in \mathcal{E}_{1}^{\prime}(k)$. This induction argument justifies the claim.

Now Theorem 7.4.1 follows from Lemma 7.3.11 and Claims 1, 2 and 3. 


\section{Chapter 8}

\section{Minimax Properties of Some Density Measures in Graphs and Digraphs}

\subsection{Introduction}

A digraph $D$ is strong if $D$ is strongly connected. A strong component of a digraph $D$ is a maximal strong subdigraph of $D$. A strong component $H$ of $D$ is nontrivial if $|A(H)|>0$. Following [11], a digraph $D$ is strict if $D$ has no loops nor parallel arcs. Throughout this chapter, we use the notation $(u, v)$ to denote an arc oriented from $u$ to $v$ in a digraph, and $[u, v]$ to denote an arc which in $\{(u, v),(v, u)\}$. A digraph $D$ is complete if $D$ is strict and for every pair $u, v$ of distinct vertices of $D$, both $(u, v)$ and $(v, u) \in A(D)$. The complete digraph on $n$ vertices will be denoted by $K_{n}^{*}$. It is known that (see Page 16 of [9], for example) for any integer $n>1, \kappa\left(K_{n}^{*}\right)=n-1$.

Using the notation in [11] and [9], for any disjoint subsets $X, Y \subseteq V(G)$, define

$$
(X, Y)_{G}=\{x y \in E(G): x \in X, y \in Y\} \text { and } \partial_{G}(X)=(X, V(G)-X)_{G}
$$

When $X=\{v\}$, we often use $\partial_{G}(v)$ for $\partial_{G}(\{v\})$. Likewise, for any disjoint subsets $X, Y \subseteq$ $V(D)$, define

$$
\begin{gathered}
(X, Y)_{D}=\{(x, y) \in A(D): x \in X, y \in Y\}, \partial_{D}^{+}(X)=(X, V(D)-X)_{D} \\
\text { and } \partial_{D}^{-}(X)=\partial_{D}^{+}(V(D)-X) .
\end{gathered}
$$

For each $v \in V(D)$, we use $\partial_{D}^{+}(v)$ for $\partial_{D}^{+}(\{v\})$ and $\partial_{D}^{-}(v)$ for $\partial_{D}^{-}(\{v\})$. The out-degree (in-degree, respectively) of $v$ in $D$, is $d_{D}^{+}(v)=\left|\partial_{D}^{+}(v)\right|\left(d_{D}^{-}(v)=\left|\partial_{D}^{-}(v)\right|\right.$, respectively). We also define

$$
N_{D}^{+}(v)=\{u \in V(D):(v, u) \in A(D)\} \text { and } N_{D}^{-}(v)=\{u \in V(D):(u, v) \in A(D)\}
$$


For a graph $G$, let $f(G)$ denote the edge-connectivity $\kappa^{\prime}(G)$ or the minimum degree $\delta(G)$ of $G$, and define $\bar{f}(G)=\max \{f(H): H$ is a subgraph of $G\}$. As indicated in [48], networks modeled as a graph $G$ with $f(G)=\bar{f}(G)$ are of particular interest of investigations. Matula first studied the quantities

$$
\bar{\kappa}^{\prime}(G)=\max \left\{\kappa^{\prime}(H): H \subseteq G\right\} \text { and } \bar{\delta}(G)=\max \{\delta(H): H \subseteq G\} .
$$

These graph invariants have drawn the attention of researchers as early as in the 1960s. Graphs $G$ with $\bar{\delta}(G) \leq k$ are called $k$-degenerate graphs and were first investigated in [63]. For any fixed integer $k>0$, the $k$-core of a graph $G$ is the unique maximal subgraph $H$ of $G$ with $\delta(H) \geq k$, and can be obtained from $G$ by repeatedly deleting vertices of degree less than $k$. The $k$-cores are considered as fundamental structures in graph theory, as seen in [28, 29, 72, 81, 87], among others. Weighted version of $k$-cores is introduced in [40] to study the communities cooperation level in social science. Other social network applications can be found in [83]. As commented in [50, 72, 79], both $\bar{\delta}(G)$ and $\bar{\kappa}^{\prime}(G)$ are related to graph coloring problems.

In order to compute $\bar{\kappa}^{\prime}(G)$ and $\bar{\delta}(G)$, Matula defined slicings.

Definition 8.1.1. Let $G$ be a graph with $E(G) \neq \emptyset$.

(i) A sequence of disjoint nonempty edge subsets $Z=\left(J_{1}, J_{2}, \ldots, J_{m}\right)$ is a slicing of $G$ if $J_{1}$ is an edge cut of $G$, and for each $i$ with $2 \leq i \leq m, J_{i}$ is an edge cut of $G-\bigcup_{j=1}^{i-1} E\left(J_{j}\right)$.

(ii) If there exists a sequence $v_{1}, v_{2}, \ldots, v_{m}$ of vertices of $G$ such that $J_{1}=\partial_{G}\left(v_{1}\right)$ and for $i \geq 2$, $J_{i}=\partial_{G-\bigcup_{j=1}^{i-1} E\left(J_{j}\right)}\left(v_{i}\right)$, then the slicing $Z=\left(J_{1}, J_{2}, \ldots, J_{m}\right)$ is a $\delta$-slicing of $G$. (iii) If $Z=\left(J_{1}, J_{2}, \ldots, J_{m}\right)$ is a slicing of $G$, then the width of $Z$, is

$$
w(Z)=\max \left\{\left|J_{i}\right|: 1 \leq i \leq m\right\}
$$

In [74], Matula discovered some minimax results involving $\bar{\kappa}^{\prime}(G)$ and $\bar{\delta}(G)$.

Theorem 8.1.2. (Matula [74]) For any graph $G$ with $|E(G)| \geq 1$, each of the following holds. (i) $\bar{\kappa}^{\prime}(G)=\max \left\{\kappa^{\prime}(H): H \subseteq G\right\}=\min \{w(Z): Z$ is a slicing of $G\}$.

(ii) $\bar{\delta}(G)=\max \{\delta(H): H \subseteq G\}=\min \{w(Z): Z$ is a $\delta$-slicing of $G\}$.

While the parameters $\bar{\delta}(G), \bar{\kappa}^{\prime}(G)$ and $\bar{\kappa}(G)$ have been intensively studied, to the best of our knowledge, the related problem on the other network reliability measures and the corresponding measures of digraphs have rarely investigated. The purpose of this chapter is to investigate whether digraphs will have similar behaviors, and to seek if Theorem 8.1 .2 can be extended to other graph reliability measures. As in [9], the minimum out-degree and the minimum in-degree of a digraph $D$ are $\delta^{+}(D)=\min \left\{d_{D}^{+}(v): v \in V(D)\right\}$ and $\delta^{-}(D)=\min \left\{d_{D}^{-}(v): v \in V(D)\right\}$, respectively. Naturally, for a digraph $D$, we define

$$
\bar{\lambda}(D)=\max \{\lambda(H): H \subseteq D\}, \bar{\delta}^{+}(D)=\max \left\{\delta^{+}(H): H \subseteq D\right\}
$$




$$
\text { and } \bar{\delta}^{-}(D)=\max \left\{\delta^{-}(H): H \subseteq D\right\} \text {. }
$$

Some of the recent studies on $\lambda(D)$ and $\bar{\lambda}(D)$ focused on extremal properties and the relationship with arc disjoint spanning arborescences, as seen in $[5,6,61,68]$, among others. By the definition of $\bar{\lambda}(D)$, we observe that $\bar{\lambda}(D)=0$ if and only if $D$ does not contain a directed cycle. That is, $D$ is acyclic. Therefore, throughout this chapter, when discussing $\bar{\lambda}(D)$, we always assume that $\lambda(D)>0$.

A natural model for digraph slicing will be a sequence of disjoint and nonempty arc subsets in the form $\partial_{D_{i}}^{+}(X)$ for some subdigraph $D_{i}$ of $D$. Similarly, $\delta^{+}$-slicings $\left(\delta^{-}\right.$-slicings, respectively) will be sequences of disjoint nonempty arc subsets in the form $\partial_{D_{i}}^{+}(v)\left(\partial_{D_{i}}^{-}(v)\right.$, respectively). We observe that in a nontrivial graph $G$, every edge lies in an edge cut of the connected component of $G$ containing the edge. But in a nontrivial digraph $D$, not every arc is lying in a directed cut of a strong component of $D$. Therefore, we would need to modify the definition of a graph slicing to define a digraph slicing to accommodate this difference, and the proving arguments would also be altered accordingly. In their studies of fault tolerance networks, Esfahanian and Hakimi in $[30,31]$ introduced restricted edge-connectivity of a graph. An edge cut $X$ of a graph $G$ is restricted if for any $v \in V(G), \partial_{G}(v)-X \neq \emptyset$. The restricted edge-connectivity of a graph $G$, denoted by $\lambda_{2}(G)$, is the minimum size of a restricted edge-cut of $G$. The concept of different slicing will be formally defined in the next section.

In the next section, we present the results for these minimax relations. In the last section, we will develop the concept of $\lambda_{2}$-slicing of $G$ and prove an analogous minimax duality result that determines the value of $\overline{\lambda_{2}}(G)=\max \left\{\lambda_{2}(H): H \subseteq G\right\}$.

Our approaches to the digraph generalization of Theorem 8.1.2 are motivated by and similar to the work of Matula in $[72,73,74,75]$. The minimax theorem on the restricted edgeconnectivity of graph is also motivated by these results.

\subsection{Slicing of Digraphs}

Let $k \geq 0$ be an integer. A digraph $D$ is $k$-arc-strong if $\lambda(D) \geq k$, or equivalently, for any proper nonempty subset $\emptyset \neq X \subset V(D)$, we always have $\left|\partial_{D}^{+}(X)\right| \geq k$. Thus in this sense, every digraph $D$ is 0 -arc-strong, and $\lambda(D)=0$ if and only if $D$ is not 1-arc-strong. Let $D$ be a digraph and let $D_{1}$ and $D_{2}$ be two subdigraphs of $D$. Define $D_{1} \cup D_{2}$ to be the subdigraph of $D$ with $V\left(D_{1} \cup D_{2}\right)=V\left(D_{1}\right) \cup V\left(D_{2}\right)$ and $A\left(D_{1} \cup D_{2}\right)=A\left(D_{1}\right) \cup A\left(D_{2}\right)$. We start with some elementary properties. Proposition 8.2.1 below follows by an argument similar to that by Matula in $[72,73]$.

Proposition 8.2.1. (Anderson et al. , [5]) Let $D_{1}, D_{2}, \ldots, D_{n}$ be subdigraphs of a digraph $D$ such that $\bigcup_{i=1}^{n} D_{i}$ is strongly connected. Then $\lambda\left(\bigcup_{i=1}^{n} D_{i}\right) \geq \min _{1 \leq i \leq n} \lambda\left(D_{i}\right)$. 
It follows from the definitions that for any strong digraph $D$,

$$
\kappa(D) \leq \lambda(D) \leq \min \left\{\delta^{+}(D), \delta^{-}(D)\right\}
$$

Proposition 8.2.2. (Anderson et al. , [5, 7]) Let $D$ be a strong digraph. Then $\bar{\kappa}(D) \leq \bar{\lambda}(D) \leq$ $\min \left\{\bar{\delta}^{+}(D), \bar{\delta}^{-}(D)\right\}$.

We assume that $D$ is a digraph with $\bar{\lambda}(D)>0$. An arc subset $W$ of $D$ is a direct cut of $D$ if there exists a nonempty proper vertex subset $X$ such that $W=(X, V(D)-X)_{D}$ with $W \neq \emptyset$. We present a formal definition of digraph slicing below.

Definition 8.2.3. Let $D$ be a digraph with $\lambda(D)>0$. Set $D_{1}=D$.

(i) A slicing of $D$ is a sequence $S=\left(J_{1}, J_{2}, \ldots, J_{s}\right)$ of arcs subsets of $D$ with $s \geq 2$ such that each of the following holds.

(i-1) $J_{1}$ is a direct cut of $D_{1}$.

(i-2) Define $D_{2}=D-J_{1}$. For $i=2,3, \ldots, s-1, D_{i}$ is not acyclic, $J_{i}$ is a nonempty direct cut of $D_{i}$ and set $D_{i+1}=D_{i}-J_{i}$.

(i-3) $D_{s}=D-\bigcup_{i=1}^{s-1} J_{i}$ is acyclic.

(ii) If for each $i$ with $1 \leq i \leq s-1, J_{i}$ is a minimum direct cut of a nontrivial strong component of $D_{i}$, then the slicing $S=\left(J_{1}, J_{2}, \ldots, J_{s}\right)$ is a narrow slicing.

(iii) The width of a slicing $S=\left(J_{1}, J_{2}, \ldots, J_{s}\right)$ is $w(S)=\max \left\{\left|J_{i}\right|, 1 \leq i \leq s-1\right\}$.

(iv) The collection of all slicings of $D$ is denoted by $\mathcal{S}(D)$.

We assume that $D$ is a digraph with $A(D) \neq \emptyset$. For a digraph $D$, let $G(D)$, called the underlying graph of $D$, be the graph obtained from $D$ be erasing all the orientation of the arcs of $D$. A digraph $D$ is weakly connected if $G(D)$ is connected. A subdigraph $H$ of $D$ is a weak component of $D$ if $G(H)$ is a component of $G(D)$ with $|A(H)|>0$. (Thus, an isolated vertex of $D$ is not a weak component.) Similarly, we start with a formal definition of a $\delta^{+}$-slicing, as well as one of a $\delta^{-}$-slicing.

Definition 8.2.4. Let $D$ be a digraph with $A(D) \neq \emptyset$.

(i) A sequence of disjoint arc subsets $S=\left(J_{1}, J_{2}, \ldots, J_{s}\right)$ of $D$ is a $\delta^{+}$-slicing (or $\delta^{-}$-slicing, respectively) of $D$ if each $J_{i} \neq \emptyset, 1 \leq i \leq s$, and if each of the following holds:

$(i-1)$ Let $D_{1}=D$. There exists a vertex $v_{1} \in V\left(D_{1}\right)$ such that $J_{1}=\partial_{D_{1}}^{+}\left(v_{1}\right)\left(J_{1}=\partial_{D_{1}}^{-}\left(v_{1}\right)\right.$, respectively).

(i-2) For $i=2, \ldots, s$, set $D_{i}=D_{i-1}-J_{i-1}$, and there exists a vertex $v_{i} \in V\left(D_{i}\right)$ such that $J_{i}=\partial_{D_{i}}^{+}\left(v_{i}\right)\left(J_{i}=\partial_{D_{i}}^{-}\left(v_{i}\right)\right.$, respectively).

(i-3) $A\left(D_{s}\right)-J_{s}=\emptyset$.

(ii) $A \delta^{+}$-slicing (or a $\delta^{-}$-slicing, respectively) $S=\left(J_{1}, J_{2}, \ldots, J_{s}\right)$ is minimal if for each $i$ with 
$1 \leq i \leq s$, there exists a weak component $L_{i}$ of $D_{i}$ such that $\left|J_{i}\right|=\delta^{+}\left(L_{i}\right) \quad\left(\right.$ or $\left|J_{i}\right|=\delta^{-}\left(L_{i}\right)$, respectively).

(iii) Let $\mathcal{S}^{+}(D)$ and $\mathcal{S}^{-}(D)$ denote the collections of all $\delta^{+}$-slicings and all $\delta^{-}$-slicings of $D$, respectively.

By Definition 8.2.4, if $S=\left(J_{1}, J_{2}, \ldots, J_{m}\right)$ is a $\delta^{+}$-slicing of $D$, then for each $i=1,2, \ldots, m$, there exists a weak component $D_{i}^{\prime}$ of $D_{i}$ and a vertex $v_{i} \in V\left(D_{i}^{\prime}\right)$ such that $J_{i}=\partial_{D_{i}^{\prime}}^{+}\left(v_{i}\right)$.

Theorem 8.2.5. (Anderson et al. , [5, 7]) Let $D$ be a digraph with $A(D) \neq \emptyset$. Let $\mathcal{S}(D)$ be the collection of all slicings of $D$ and let $\mathcal{S}^{+}(D), \mathcal{S}^{-}(D)$ be the collection of all $\delta^{+}$-slicings of $D$ and all $\delta^{-}$-slicings of $D$, respectively. Each of the following holds.

(i) Assume that $\bar{\lambda}(D)>0$. Then $\max \left\{\min \left\{\left|\partial_{H}^{+}(X)\right|: \emptyset \neq X \subset V(H)\right\}: H \subseteq D\right\}=$ $\min \left\{\max \left\{\left|J_{i}\right|: 1 \leq i \leq m\right\}: S=\left(J_{1}, J_{2}, \ldots, J_{m}\right) \in \mathcal{S}(D)\right\}$.

(ii) $\max \left\{\min \left\{d_{H}^{+}(v): v \in V(H)\right\}: H \subseteq D\right\}=\min \left\{\max \left\{\left|J_{i}\right|: 1 \leq i \leq m\right\}: S=\left(J_{1}, J_{2}, \ldots, J_{m}\right) \in\right.$ $\left.\mathcal{S}^{+}(D)\right\}$.

(iii) $\max \left\{\min \left\{d_{H}^{-}(v): v \in V(H)\right\}: H \subseteq D\right\}=\min \left\{\max \left\{\left|J_{i}\right|: 1 \leq i \leq m\right\}: S=\right.$ $\left.\left(J_{1}, J_{2}, \ldots, J_{m}\right) \in \mathcal{S}^{-}(D)\right\}$

There are some computational useful ways of determining $\bar{\delta}^{+}(D)$ and $\bar{\delta}^{-}(D)$, as stated in the following results.

Lemma 8.2.6. (Anderson et al. , [5, 7]) Let $D$ be a digraph with $A(D) \neq \emptyset$.

(i) If $S=\left(J_{1}, J_{2}, \ldots, J_{s}\right)$ is a minimal $\delta^{+}$-slicing of $D$, then

$$
\bar{\delta}^{+}(D)=\max _{1 \leq i \leq s}\left\{\left|J_{i}\right|\right\}
$$

(ii) If $S=\left(J_{1}, J_{2}, \ldots, J_{s}\right)$ is a minimal $\delta^{-}$-slicing of $D$, then

$$
\bar{\delta}^{-}(D)=\max _{1 \leq i \leq s}\left\{\left|J_{i}\right|\right\}
$$

\subsection{A Minimax Theorem in Restricted Edge-connectivity}

In their studies of fault tolerance networks, Esfahanian and Hakimi in [30, 31] introduced restricted edge-connectivity of a graph. There has been intensive researches on restricted edgeconnectivity, as seen in the recent survey of Xu [96]. An edge cut $X$ of a graph $G$ is restricted if for any $v \in V(G), \partial_{G}(v)-X \neq \emptyset$. With this definition, not every connected graph may have a restricted edge-cut. Let $\mathcal{F}$ be a family of connected graph such that a graph $G$ is in $\mathcal{F}$ if and only if either $G$ is spanned by a $K_{3}$, or $G$ has a vertex $v \in V(G)$ such that $E(G-v)=\emptyset$.

Lemma 8.3.1. Let $G$ be a connected graph with $|E(G)|>0$. Then $G$ does not have a restricted edge-cut if and only if $G \in \mathcal{F}$. 
Proof. Let $G$ be a connected graph with $|E(G)|>0$ which does not have a restricted edgecut. Since every graph on 2 vertices must be in $\mathcal{F}$, we assume that $|V(G)| \geq 3$. Assume that $|V(G)|=3$ and $G$ is not spanned by a $K_{3}$, then $G$ has a cut vertex $v$, and so $E(G-v)=\emptyset$, whence $G \in \mathcal{F}$. Thus we assume that $|V(G)| \geq 4$. If $G$ has a path of length at least 3 , then $G$ has a restricted edge-cut. Hence every longest path of $G$ has length 2 . Since $|V(G)| \geq 4, G$ cannot have a cycle of length at least 3 . It follows that $G$ must be spanned by a $K_{1, n-1}$, where $n=|V(G)|$. Since $G$ contains no cycles of length at least 3, if $v \in V(G)$ has maximum degree in $G$, then $E(G-v)=\emptyset$, and so $G \in \mathcal{F}$. Conversely, it follows by definition that every member in $\mathcal{F}$ does not have a restricted edge-cut.

Lemma 8.3.1 indicates that in order to define restricted edge-connectivity of a graph, we need to define restricted edge-cuts of graphs in $\mathcal{F}$. To facilitate the study of restricted edgeconnectivity of a graph, we further define that for any $G \in \mathcal{F}$, we define an edge subset $X \subseteq E(G)$ such that it is a restricted edge-cut of $G$ if and only if $|X|=|E(G)|-1$. The restricted edgeconnectivity of a nontrivial connected graph $G$, denoted by $\lambda_{2}(G)$, is the minimum size of a restricted edge-cut of $G$. Note that $\lambda_{2}\left(K_{2}\right)=0$. If $G=K_{1}$ or if $G$ is not connected, it is natural to define that $\lambda_{2}(G)=0$. In this section, we will develop the concept of $\lambda_{2}$-slicing of $G$ and prove an analogous minimax duality result that determines the value of $\overline{\lambda_{2}}(G)=\max \left\{\lambda_{2}(H): H \subseteq G\right\}$.

\subsubsection{Restricted Slicing of a Graph}

Let $G$ be a connected graph such that $G \notin \mathcal{F}$. A restricted edge cut $S$ of $G$ is minimal if it contains no other restricted edge-cut of $G$. Thus if $S$ is a minimal restricted edge-cut of $G$, then $G-S$ has exactly two nontrivial connected components $G^{\prime}, G^{\prime \prime}$. If $G \in \mathcal{F}$, then for any restricted edge-cut $S$ of $G, G-S$ has exactly one nontrivial component isomorphic to $K_{2}$. We start with a lemma below.

Lemma 8.3.2. Let $G$ be a nontrivial connected graph such that $G \notin \mathcal{F}$. If $S$ is a minimal restricted edge-cut of $G$ such that $G-S$ has components $G^{\prime}, G^{\prime \prime}$, then

$$
\overline{\lambda_{2}}(G)=\max \left\{|S|, \overline{\lambda_{2}}\left(G\left[E\left(G^{\prime}\right) \cup S\right]\right), \overline{\lambda_{2}}\left(G\left[E\left(G^{\prime \prime}\right) \cup S\right]\right)\right\}
$$

Proof. By definition, there exists a connected subgraph $H$ of $G$ such that $\lambda_{2}(H)=\overline{\lambda_{2}}(G)$. Since $S$ is a minimal, by definition, we have $H=G$ if and only if $\overline{\lambda_{2}}(G)=|S|$.

Assume first that $H=G$, or equivalently, $\overline{\lambda_{2}}(G)=|S|$. Then by definition of $\overline{\lambda_{2}}(G)$, we have $\overline{\lambda_{2}}(G) \geq \max \left\{\overline{\lambda_{2}}\left(G\left[E\left(G^{\prime}\right) \cup S\right]\right), \overline{\lambda_{2}}\left(G\left[E\left(G^{\prime \prime}\right) \cup S\right]\right)\right\}$. Hence (8.2) holds. Now assume that $H \neq G$. Thus $\overline{\lambda_{2}}(G)>|S|$. If $H$ is a subgraph of $G\left[E\left(G^{\prime}\right) \cup S\right]$, then $\overline{\lambda_{2}}(G)=\lambda_{2}(H)=$ $\overline{\lambda_{2}}\left(G\left[E\left(G^{\prime}\right) \cup S\right]\right) \geq \max \left\{|S|, \overline{\lambda_{2}}\left(G\left[E\left(G^{\prime \prime}\right) \cup S\right]\right)\right\}$, whence (8.2) holds. Thus it suffices to show that either $\left.H \subseteq G\left[E\left(G^{\prime}\right) \cup S\right]\right)$ or $\left.H \subseteq G\left[E\left(G^{\prime \prime}\right) \cup S\right]\right)$. 
By contradiction, we assume that $H$ is not a subgraph of $\left.G\left[E\left(G^{\prime}\right) \cup S\right]\right)$ and $H$ is a subgraph of $\left.G\left[E\left(G^{\prime \prime}\right) \cup S\right]\right)$. These imply that $E(H) \cap E\left(G^{\prime}\right) \neq \emptyset$ and $E(H) \cap E\left(G^{\prime \prime}\right) \neq \emptyset$. It follows that $S \cap E(H)$ is a restricted edge-cut of $H$, and so

$$
\overline{\lambda_{2}}(G)>|S| \geq|S \cap E(H)| \geq \lambda_{2}(H)=\overline{\lambda_{2}}(G),
$$

showing that a contradiction obtains. This contradiction justifies that either $\left.H \subseteq G\left[E\left(G^{\prime}\right) \cup S\right]\right)$ or $\left.H \subseteq G\left[E\left(G^{\prime \prime}\right) \cup S\right]\right)$, and so (8.2) must hold.

We will define the $\lambda_{2}$-slicing of a connected graph $G$. To do that, we introduce a subroutine as follows.

Subroutine $\Phi(\Gamma, S, F)$.

Input. a graph $\Gamma$ with nontrivial connected components $H_{1}, H_{2}, \ldots, H_{t}$. Initially set $S=\emptyset$ and $F=\left\{H_{1}, H_{2}, \ldots, H_{t}\right\}$.

(S1) Choose $H \in F$ such that $|E(H)|=\max \left\{E\left(H_{j}\right) \mid: 1 \leq j \leq t\right\}$. If $|E(H)| \leq 1$, then set $S=\emptyset$ and stop.

(S2) Assume that $|E(H)|>1$.

(S2-1) If $H \in F$, then pick any $e_{H} \in E(H)$, set $S=E(H)-\left\{e_{H}\right\}$ and $F:=F-\{H\}$.

(S2-2) If $H \notin F$, then find a restricted edge-cut $S$ of $G$. Let $G^{(1)}, G^{(2)}, \ldots, G^{(s)}$ be the nontrivial components of $G-S$. Define, for $1 \leq i \leq s, H_{i}^{\prime}=G\left[E\left(G^{(i)}\right) \cup S\right]$. Set $S:=S$, $F:=(F-\{H\}) \cup\left\{H_{1}^{\prime}, H_{2}^{\prime}, \ldots, H_{s}^{\prime}\right\}$.

Output. An edge subset $S$ of $\Gamma$ such that either $S=\emptyset$, or $S$ is a restricted edge-cut of $G$, as well as a collection $F$ of graphs, each of which is isomorphic to a subgraph of $\Gamma$.

With Subroutine $\Phi(\Gamma, S, F)$, we have the following algorithm that generate the $\lambda_{2}$-slicings of $G$. Given a connected graph $G$.

Algorithm Slicing. Let $G$ be a connected graph with $G \notin\left\{K_{1}, K_{2}\right\}$. Initially, we first set $G_{0}=G, F_{0}=\left\{G_{0}\right\}$ and set $\sigma$ to be the empty sequence.

Apply Subroutine $\Phi\left(G_{0}, S_{1}, F_{1}\right)$. If the output $S_{1}=\emptyset$, then stop and we conclude that $G \in\left\{K_{1}, K_{2}\right\}$, and so $\lambda_{2}(G)=\overline{\lambda_{2}}(G)=0$. If $S_{1} \neq 0$, the Subroutine $\Phi\left(G_{0}, S_{1}, F_{1}\right)$ outputs a restricted edge-cut $S_{1}$ of $G_{0}$ and a collection $F_{1}$ of graphs such that each of which is isomorphic to a subgraph of $G$. Update $\sigma=\left(S_{1}\right)$ as a one term sequence, and define $G_{1}$ to be the graph whose connected components are precisely those graphs in $F_{1}$. Thus up to isomorphism, graphs in $F_{1}$ are subgraphs of $G$.

Inductively, assume that $\sigma=\left(S_{1}, S_{2}, \ldots, S_{k}\right)$ and the graph $G_{k}$ are found. We then apply Subroutine $\Phi\left(G_{k}, S_{k+1}, F_{k+1}\right)$. If the output $S_{k+1}=\emptyset$, then stop, and we define the current value $\sigma$ is a $\lambda_{2}$-slicing of $G$. Otherwise, $S_{k+1} \neq \emptyset$, and we update $\sigma:=\left(S_{1}, S_{2}, \ldots, S_{k}, S_{k+1}\right)$, and define $G_{k+1}$ to be the graph whose connected components are precisely those graphs in $F_{k+1}$.

We shall show that this algorithm terminates in finite time so that if a connected graph $G \notin\left\{K_{1}, K_{2}\right\}$, then the algorithm will generate a $\lambda_{2}$-slicing of $G$. For each current value $F^{\prime}=$ 
$\left\{H_{1}, H_{2}, \ldots, H_{t}\right\}$, we assume that $\left|E\left(H_{1}\right)\right| \geq\left|E\left(H_{2}\right)\right| \geq \ldots \geq E\left(H_{t}\right)$. Let $f\left(F^{\prime}\right)=\{i: 1 \leq i \leq t\}$ and $\left|E\left(H_{i}\right)\right|=\max \left\{\left|E\left(H_{j}\right)\right|, 1 \leq j \leq t\right\}$. After one application of $\Phi(G, S, F)$, without lose of generality, we assume that $H_{1}$ is picked by the subroutine. In the execution of (S2-1), $H_{1}$ will be removed from the output $F$; in the execution of (S2-2), as each of the new edge induced subgraphs has number of edges less than $\left|E\left(H_{1}\right)\right|$, we conclude that $f\left(F^{\prime}\right)>f(F)$. As $f(F)$ is integral and as each time running the subroutine $\Phi(G, S, F)$, the output value $f(F)$ is strictly less then the input value. The algorithm must terminate in a finite time. For a connected graph $G$, let $\sigma(G)$ denote the collection of all $\lambda_{2}$-slicings of $G$.

In the execution of Subroutine $\Phi(G, S, F)$, we do not require, in Step (S2-2), that the restricted edge cut $S$ to be a minimum one. We now define a similar Subroutine $\Phi^{\prime}(G, S, F)$ by additionally requiring that in the execution of (S2-2) of the Subroutine $\Phi^{\prime}(G, S, F)$, the restricted edge-cut $S$ must be minimized. With this new subroutine $\Phi^{\prime}(G, S, F)$, we again run the algorithm described above to generate $\lambda_{2}$-slicing of $G$. These slicings will be called the restricted narrow slicing or narrow $\lambda_{2}$-slicing of $G$. Let $\sigma^{\prime}(G)$ denote the set of all narrow $\lambda_{2}$-slicings of $G$.

Lemma 8.3.3. Let $G$ be a connected graph not in $\left\{K_{1}, K_{2}\right\}$ and let $\sigma=\left(S_{1}, S_{2}, \ldots, S_{s}\right) \in \sigma(G)$. If $H$ is a subgraph of $G$ satisfying $\lambda_{2}(H)=\overline{\lambda_{2}}(G)$, then for some $j$ with $1 \leq j \leq s, S_{j}$ is a restricted edge-cut of $H$.

Proof. We argue by induction on $|V(G)-V(H)|$. If $V(G)=V(H)$, then $G=H$ and so as $S_{1}$ is a restricted edge-cut of $G, S_{1}$ is a restricted edge-cut of $H$. Let $h$ be the smallest integer with $1 \leq h \leq s$ such that $S_{h} \cap E(H) \neq \emptyset$.

If $S_{h}$ is a restricted edge-cut of $H$, then the lemma is proved. Assume that $S_{h}$ is not a restricted edge-cut of $H$. Then by Lemma 8.3.2 and by Algorithm Slicing, there must be a graph $H^{\prime} \in F_{h}$ such that $H$ is a subgraph of $H^{\prime}$, with $\left|V\left(H^{\prime}\right)\right|<|V(G)|$. By induction, there must be an index $j$ with $h \leq j \leq s$ such that $S_{j}$ is a restricted edge-cut of $H$.

\subsubsection{A Minimax Theorem of Restricted Edge-connectivity}

Throughout out this subsection, $G$ is assumed to be a connected graph not in $\left\{K_{1}, K_{2}\right\}$. The main result of this section is the following minimax result.

Theorem 8.3.4. Let $G$ be a connected graph not in $\left\{K_{1}, K_{2}\right\}$. Then

$$
\begin{aligned}
\overline{\lambda_{2}}(G) & =\max _{H \subseteq G} \min \{|X|: X \text { is a restricted edge-cut of } H\} \\
& =\min _{\sigma \in \sigma(G)} \max \left\{\left|S_{i}\right|: 1 \leq i \leq s, \sigma=\left(S_{1}, S_{2}, \ldots, S_{s}\right)\right\}
\end{aligned}
$$

Proof. Let $\ell=\min _{\sigma \in \sigma(G)} \max \left\{\left|S_{i}\right|: 1 \leq i \leq s, \sigma=\left(S_{1}, S_{2}, \ldots, S_{s}\right)\right\}$. We shall show that both $\overline{\lambda_{2}}(G) \leq \ell$ and $\overline{\lambda_{2}}(G) \geq \ell$. By definition, there exists a nontrivial subgraph $H$ of $G$ such that $\overline{\lambda_{2}}(G)=\lambda_{2}(H)$. 
For any $\sigma=\left(S_{1}, S_{2}, \ldots, S_{s}\right) \in \sigma(G)$, by Lemma 8.3.3, there must be an index $j$ with $1 \leq j \leq s$, $S_{j}$ is a restricted edge-cut of $H$. It follows that $\max \left\{\left|S_{i}\right|: 1 \leq i \leq s, \sigma=\left(S_{1}, S_{2}, \ldots, S_{s}\right)\right\} \geq$ $\left|S_{j}\right| \geq \lambda_{2}(H)=\overline{\lambda_{2}}(G)$. Since $\sigma \in \sigma(G)$ is arbitrary, we must have $\ell \geq \overline{\lambda_{2}}(G)$.

Conversely, let $\sigma=\left(S_{1}, S_{2}, \ldots, S_{s}\right) \in \sigma^{\prime}(G)$ be a narrow $\lambda_{2}$-slicing. By Algorithm Slicing, in each iteration, each graph $H^{\prime}$ in the the resulting collection of subgraphs $F_{i}$ is isomorphic to a subgraph of $G$. Thus by the definition of a narrow $\lambda_{2}$-slicing, each $S_{i}$ is a minimum restricted edge-cut of some subgraph of $G$, and so $\overline{\lambda_{2}}(G) \geq\left|S_{i}\right|$ for each $i$ with $1 \leq i \leq s$. It follows that

$$
\overline{\lambda_{2}}(G) \geq \min _{\sigma \in \sigma^{\prime}(G)} \max \left\{\left|S_{i}\right|: 1 \leq i \leq s, \sigma=\left(S_{1}, S_{2}, \ldots, S_{s}\right)\right\} \geq \ell .
$$

This completes the proof of the theorem.

\subsection{Future Studies}

We have observed that, for a number of density measures $f$ of graphs and digraphs, there exist

minimax theorems to determine $\bar{f}$, as seen in Theorem 8.1.2, Theorem 8.2.5 and Theorem 8.3.4. We believe that there might be a more general theorem that can cover all these as special cases and we have not found this general result yet. 


\section{Bibliography}

[1] A. Ahadi, S. Akbari, A. Dehghana, M. Ghanbari, On the difference between chromatic number and dynamic chromatic number of graphs, Discrete Mathematics 312 (2012) 25792583.

[2] S. Akbari, M. Ghanbari, S. Jahanbekam, On the list dynamic coloring of graphs, Discrete Appl. Math. 157 (2009) 3005-3007.

[3] M. Alishahi, On the dynamic coloring of graphs, Discrete Appl. Math. 159 (2011) 152-156.

[4] M. Alishahi, Dynamic chromatic number of regular graphs, Discrete Applied Mathematics 160 (2012) 2098-2103.

[5] J. Anderson, A Study of Arc Strong Connectivity of Digraphs, PhD Dissertation, West Virginia University, (2017).

[6] J. Anderson, H.-J. Lai, X. Lin and M. Xu, On k-maximal strength digraphs, J. Graph Theory, 84 (2017) 17-25.

[7] J. Anderson, H.-J. Lai, X. Li, X. Lin and M. Xu, Minimax properties of some density measures in graphs and digraphs, International Journal of Computer Mathematics, published online in 2018. https://doi.org/10.1080/23799927.2017.1410498

[8] G. Bacso and ZS. Tuza, Dominating cliques in $P_{5}$-free graphs, Periodica Mathematica Hungarica, Vol. 21 (4), 1990, pp. 303-308.

[9] J. Bang-Jensen and G. Gutin, Digraphs: Theory, Algorithms and Applications, 2nd Edition, Springer-Verlag, London, 2009.

[10] V. Batagelj and M. Zaversnik, An $O(m)$ algorithm for cores decomposition of networks, arXiv preprint cs/0310049, 2003.

[11] J. A. Bondy and U. S. R. Murty, Graph Theory. Springer, New York, 2008. 
[12] Y. H. Bu, X. B. Zhu, An optimal square coloring of planar graphs, J Comb Optim (2012) 24:580 - 592 .

[13] M. Burati, A. Del Fra, Cyclic Hamiltonian cycle systems of the complete graph, Discrete Math., 279 (2004), 107-119.

[14] C. Cai, D. Xie, W. Yang, A result on linear coloring of planar graphs, Inform. Process. Lett. 112 (2012) 880-884.

[15] P. A. Catlin, J. W. Grossman, A. M. Hobbs and H.-J. Lai, Fractional arboricity, strength, and principal partition in graphs and matroids. Discrete Appl. Math., 40 (1992) 285-302.

[16] P. A. Catlin, H.-J. Lai and Y. H. Shao, Edge-connectivity and edge-disjoint spanning trees, Discrete Math., 309 (2009) 1033-1040.

[17] G. Chartrand, D. P. Geller and S. T. Hedetniemi, Graphs with forbidden subgraphs, J. Combin. Th., 10 (1971) 12-41.

[18] C. C. Chen, K. M. Koh and Y. H. Peng, On the higher-order edge toughness of a graph, Discrete Math., 111 (1993) 113-123.

[19] Y. Chen, S. Fan, H.-J. Lai, H. Song and L. Sun, On dynamic coloring for planar graphs and graphs of higher genus, Discrete Appl. Math., 160(7-8) (2012) 1064-1071.

[20] Z. H. Chen and H.-J. Lai, The higher-order edge toughness of a graph and truncated uniformly dense matroids, J. Combin. Math. Combin. Computing, 22 (1996) 157-160.

[21] M. Chudnovsky, N. Robertson, P. D. Seymour and R. Thomas, The strong perfect graph theorem, Ann. of Math., (2) 164 (2006) 51-229.

[22] D. W. Cranston, G. Yu, Linear choosability of sparse graphs, Discrete Mathematics 311 (2011) 1910-1917.

[23] W. H. Cunningham, Optimal attack and reinforcement of a network, J. ACM , 32 (1985) 549-561.

[24] C. Ding, S. H. Fan, and H.-J. Lai, Upper bound on conditional chromatic number of graphs, J. of Jinan University 29 (2008) 7-14.

[25] W. Dong, W. Lin, On linear coloring of planar graphs with small girth, Discrete Applied Mathematics 173 (2014) 35-44.

[26] R.J. Duffin, Topology of series-parallel networks, J. Math. Anal. Appl. 10 (1965) 303-318. 
[27] J. Edmonds, Edge-dsjoint branchings, in Combinatorial Algorithms, ed. R. Rustin, Academic Press, New York, pp. 91-96, 1973.

[28] P. Erdös, On the structure of linear graphs, Israel J. Math., 1 (1963), 156-160.

[29] P. Erdös and A. Rényi, On the evolution of random graphs, Magyar Tud. Akad. Mat. Kutató Int. Közl., 5 (1960) 17-61.

[30] A. H. Esfahanian, Generalized measures of fault tolerance with application to N-cube networks, IEEE Transactions on Computers, 38 (1989) 1586-1591.

[31] A. H. Esfahanian and S. L. Hakimi, On computing a conditional edgeconnectivity of a graph. Information Processing Letters, 27 (1988), 195-199

[32] L. Esperet, Dynamic list coloring of bipartite graphs, Discrete Appl. Math. 158 (2010) 1963-1965.

[33] L. Esperet, M. Montassier, A. Raspaud, Linear choosability of graphs, Discrete Mathematics 308 (2008) 3938-3950.

[34] S. Even, Graph Algorithms, Computer Science Press, Woodland Hills, 1979.

[35] S. Fan, J. Kong, H.-J. Lai, M. Xu, Linear list $r$-hued colorings of graphs with bounded maximum average degrees, Ars Combin., (accepted for publication).

[36] A. Frank, Covering branchings, Acta Scientiarum Mathematicarum (Szeged), 41 (1979) $77-82$.

[37] H. N. Gabow, A matroid approach to finding edge-connectivity and packing arborescences, J. Comput. System Sci., 50(2) (1995) 259-273.

[38] H. N. Gabow, Using expander graphs to find vertex connectivity, J. Assoc. Comput. Mach., 53 (2006) 800-844.

[39] Z. Galil, Finding the vertex connectivity of graphs, SIAM J. Comput., 9(1), (1980) 197-199.

[40] C. Giatsidis, D. Thilikos, and M. Vazirgiannis, Evaluating cooperation in communities with the k-core structure, in Advances in Social Networks Analysis and Mining (ASONAM), 2011 International Conference on. IEEE, 2011, pp. 8793.

[41] X. Gu and H.-J. Lai Augmenting and preserving partition connectivity of a hypergraph, Journal of Combinatorics, 5 (2014) 271-289. 
[42] X. Gu, H.-J. Lai, Ping Li and S. Yao, Characterizations of minimal graphs with equal edge connectivity and spanning tree packing number, Graphs and Combinatorics, 30 (2014) 1453-1461.

[43] D. Gusfield, Connectivity and edge-disjoint spanning trees, Information Processing Letters, 16(1983), 87 - 89.

[44] H. Hadwiger, Über eine Klassifikatio der Streckomplexe, Vierteljschr. Naturforsch. Ges. Zürich, 88 (1943) 133-143.

[45] D.W. Hall, A note on primitive skew curves, Bull Amer Math Soc, 49 (1943) 932-937.

[46] J. van den Heuvel and S. Thomassé, Cyclic orderings and cyclic arboricity of matroids, Journal of Combinatorial Theory Series B, 102 (2012) 638-646.

[47] C. T. Hoang, M. Kaminski, V. Lozin, J. Sawada and X. Shu, Deciding k-colorability of $P_{5}$-free graphs in polynomial time, Algorithmica 57 (2010): 74-81.

[48] A. M. Hobbs, Survivability of networks under attack, Applications of Discrete Mathematics (eds. John G. Michaels and Kenneth H. Rosen), 1991, 332-353.

[49] S. Jahanbekam, J. Kim, Suil O, D. B. West, On r-dynamic coloring of graphs, Discrete Applied Mathematics 206 (2016) 65-72.

[50] Jensen and Toft, "Graph Coloring Problems", pp210-211. Wiley and Sons, New York, 1995.

[51] S.-J. Kim, S.-J. Lee and W.-J. Park, Dynamic coloring and list dynamic colorig of planar graphs, Discrete Applied Mathematics, 161 (2013) 2207-2212.

[52] S.-J. Kim, S. J. Lee, and S.-I. Oum, Dynamic coloring of graphs having no $K_{5}$ minor, preprint.

[53] H.-J. Lai, The size of strength-maximal graphs, J. Graph Theory, 14 (1990) 187-197.

[54] H.-J. Lai, and H. Lai. Graphs without $K_{4}$-minors, Ars Combinatoria, 36 (1993) 327-334.

[55] H.-J. Lai, H. Lai and C. Payan, A property on edge-disjoint spanning trees, Europ. J. Combinatorics, 17 (1996) 447-450.

[56] H.-J. Lai, P. Li, Y. Liang and J. Xu, Reinforcing a matroid to have $k$ disjoint bases, Applied Math., 1 (2010) 244-249.

[57] H.-J. Lai, J. Lin, B. Montgomery, T. Shui and S. Fan, Conditional Colorings of Graphs, Discrete Math. 306 (2006) 1997-2004. 
[58] H.-J. Lai, B. Montgomery, H.Poon, Upper bounds of dynamic chromatic number, Ars Combin. 68 (2003) 193-201.

[59] C. Li, W. Wang, A, Raspaud, Upper bounds on the linear chromatic number of a graph, Discrete Math., 311 (2011) 232-238.

[60] H. Li and H.-J. Lai, 3-hued coloring and list 3-hued coloring of $K_{1,3}$-free Graphs, Discrete Applied Math., 222(2017), 166-171.

[61] P. Li, H.-J. Lai and M. Xu, Disjoint Spanning Arborescences in $k$-Arc-Strong Digraphs, Ars Combinatoria, accepted.

[62] X. Li, X. Yao, W. Zhou and H. J. Broersma, Complexity of conditional colorability of graphs, Appl. Math. Letter 22 (2009) 320-324.

[63] D. R. Lick and A. T. White, k-degenerate subgraphs, Canad. J. Math. 22 (1970), 1082-1096.

[64] D. Liu, H.-J. Lai and Z.-H. Chen, Reinforcing the number of disjoint spanning trees, Ars Combin., 93 (2009) 113-127.

[65] C. Liu, G. Yu, Linear colorings of subcubic graphs, European Journal of Combinatorics, 34 (2013) 1040-1050.

[66] K. Lih, W. Wang and X. Zhu, Coloring the square of a $K_{4}$-minor free graphs, Discrete Math., 269 (2003) 303-309.

[67] Y. Lin, Upper bounds of conditional chromatics number, Master thesis, Jinan University, 2008.

[68] X. Lin, S. Fan, H.-J. Lai, and M. Xu, On the lower bound of k-maximal digraphs, Discrete Math., 339 (2016) 2500-2510.

[69] S. Loeb, T. Mahoney, B. Reiniger, and J. Wise, Dynamic coloring parameters for graphs with given genus, manuscript.

[70] E. Lucas, Recreations Mathematiques, Vol. 2, Gautheir-Villars, 1892.

[71] W. Mader, Minimale n-fach kantenzusammenhgende Graphen. Math. Ann. 191 (1971) 2128.

[72] D. W. Matula, A min-max theorem for graphs with application to graph coloring, SIAM Reviews, 10 (168) 481-482. 
[73] D. W. Matula, The cohesive strength of graphs, The Many Faces of Graph Theory, Lecture Notes in Mathematics, No. 110, G. Chartrand and S. F. Kapoor eds., Springer-Verlag, berlin, 1969, pp. 215-221.

[74] D. Matula, K-components, clusters, and slicings in graphs. SIAM J. Appl. Math. 22 (1972) 459-480.

[75] D. W. Matula, Subgraph connectivity number of a graph, Theorey and Applications of Graphs, Lecture Notes in Mathematics, No. 642, G. Chartrand and S. F. Kapoor eds., Springer-Verlag, berlin, 1976, pp. 371-383.

[76] B. Montgomery, Dynamic coloring of graphs, Ph.D. Thesis, West Virginia University, 2001.

[77] C. St. J. A. Nash-Williams, Edge-disjoint spanning trees of finite graphs, J. London Math. Soc., 36 (1961) 445-450.

[78] C. St. J. A. Nash-Williams, Decomposition of fininte graphs into forest, J. London Math. Soc., 39 (1964) 12.

[79] J. Oxley, Colouring, packing and the critical problem, Quart. J. Math. Oxford Ser. (2) 29 (1978) 11-22.

[80] C. Payan, Graphes équilibrés et arboricité rationnelle, Europ. J. Combin., 7 (1986) 263-270.

[81] B. Pitte, J. Spencer, N. Wormald, Sudden emergence of a giant $k$-core in a random graph, J. of Combinatorial Theory, Series B, 67 (1996) 111-151.

[82] C.-P. Schnorr, Bottlenecks and edge connectivity in unsymmetrical networks, SIAM J. Comput. 8(2) (1979) 265-274.

[83] S. Seidman, Network structure and minimum degree, Social networks, vol. 5, no. 3, pp. 269287, 1983.

[84] D. Seinsche, On a property of the class of $n$-colorable graphs, Journal of Combinatorial Theory, Series B., 16 (1974) 191-193.

[85] H. M. Song, S. Fan, Y. Chen, L. Sun, and H.-J. Lai, On $r$-hued coloring of $K_{4}$-minor free graphs, Discrete Mathematics, 315-316 (2014) 47-52.

[86] H. Song, H.-J. Lai and J.-L. Wu, On $r$-hued coloring of planar graphs with girth at least 6, Discrete Applied Mathematics, 198 (2016) 251-263.

[87] G. Szekeres and H. S. Wilf, An inequality for the chromatic number of a graph, J. Combinatorial Theory, 4 (1968), 1-3. 
[88] A. Taherkhani, $r$-dynamic chromatic number of graphs, Discrete Appl. Math. 201 (2016) 222-227.

[89] R. E. Tarjan, Depth-first search and linear graph algorithms, SIAM Journal of Computing 2 (1972), 146-160.

[90] T. Tillson, A Hamiltonian decomposition of $K_{2 m}^{*}$, J. Combin. Theory Ser. B, 29 (1980), 68-74.

[91] W. T. Tutte, On the problem of decomposing a graph into $n$ connected factors, J. London Math. Soc., 36 (1961) 221-230.

[92] G. Wegner. Graphs with given diameter and a coloring problem, Technical Report, University of Dortmund, 1977.

[93] D. R. Woodall, Improper colourings of graphs, in: Graph Colourings (ed. R. Nelson and R. J. Wilson), Pitman Research Notes 218, Longman (1990) 45-63.

[94] W. Wang, Y. Wang, Linear Coloring of Planar GraphsWithout 4-Cycles, Graphs and Combinatorics 29 (2013) 1113-1124.

[95] Y. Wang and Q. Wu, Linear coloring of sparse graphs, Discrete Applied Mathematics 160 (2012) 664-672.

[96] J. M. Xu, Super or Restricted Connectivity of Graphs, a survey, to appear.

[97] B. Xue, L. Zuo, G. Wang, G. Li, The Linear t-Colorings of Sierpinski-Like Graphs, Graphs and Combinatorics 30 (2014) 755-767.

[98] R. Yuster, Linear coloring of graphs, Discrete Math., 185 (1998) 293-297. 